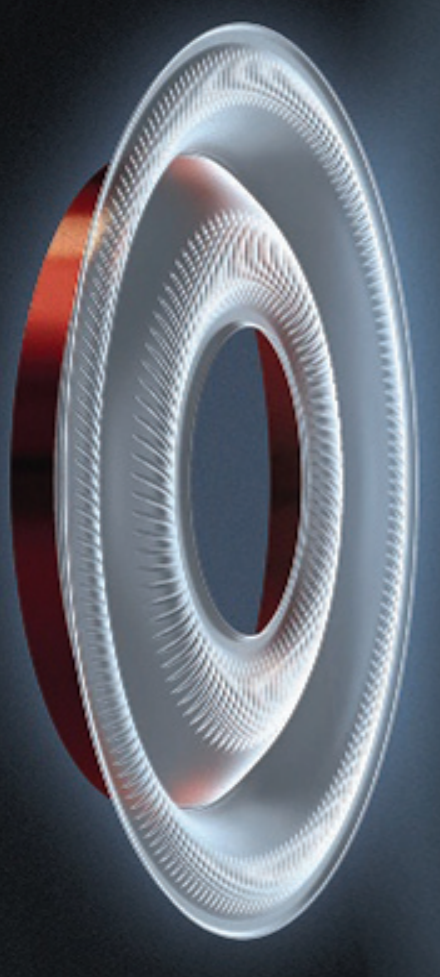

\title{
ACOUSTIC PROPERTIES OF MATERIALS
}

TEACHERS'
MANUAL





\section{MATERIALS SCIENCE PROJECT}

\author{
UNIVERSITY-SCHOOL \\ PARTNERSHIPS FOR THE DESIGN \\ AND IMPLEMENTATION OF \\ RESEARCH-BASED ICT-ENHANCED \\ MODULES ON MATERIAL \\ PROPERTIES
}

\section{SPECIFIC SUPPORT ACTIONS}

FP6: SCIENCE AND SOCIETY: SCIENCE AND EDUCATION

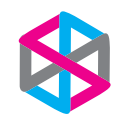

MATERIALS SCIENCE
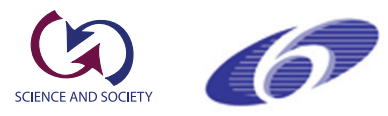

PROJECT COORDINATOR

CONSTANTINOS P. CONSTANTINOU, LEARNING IN SCIENCE GROUP, UNIVERSITY OF CYPRUS

\section{PROJECT PARTNERS}

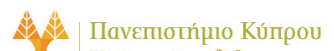
University of Cyprus

ARISTOTLE UNIVERSITY f THESSALONIKI

22. UNIVERSITY OF

Q8. WESTERN MACEDONIA

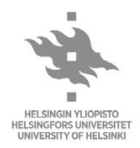

\section{UAB \\ Universitat Autònoma
de Barcelona}

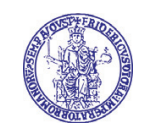

\section{ACKNOWLEDGMENT}

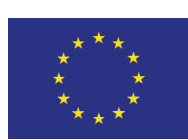

RESEARCH FUNDING FOR THE MATERIALS SCIENCE PROJECT WAS PROVIDED BY THE EUROPEAN COMMUNITY UNDER THE SIXTH FRAMEWORK SCIENCE AND SOCIETY PROGRAMME (CONTRACT SAS6-CT-2006042942).

THIS PUBLICATION REFLECTS ONLY THE VIEWS OF THE AUTHORS AND THE EUROPEAN COMMUNITY IS NOT LIABLE FOR ANY USE THAT MAY BE MADE OF THE INFORMATION CONTAINED HEREIN.

(C) DESIGN

n.eleana@cytanet.com.cy

2010, NICOSIA - CYPRUS 


\section{ACOUSTIC PROPERTIES OF MATERIALS}

Design and development

University Staff

Roser Pintó

Digna Couso

María Isabel Hernández

\section{School Teachers}

Montserrat Armengol

Celsa Cortijo

Raül Martos

Miquel Padilla

Consol Rios

Marta Simón

Carme Sunyer

Montserrat Tortosa

\section{Other contribution}

Transfer, Implementation and

Feedback

\section{Univesity Staff}

Constantinos P. Constantinou

Michalis Livitziis

Rodothea Hadjilouca

Argyro Scholinaki

Marios Papaevripidou

Nikos Papadouris

Giannis Hadjidemetriou

\section{School Teachers}

Ioannis Karmiotis

Marios Michail

Giannakis Hadjicostis

Antonis Stylianou

Nitsa Kindini

Myrto Pouangare

Leonidas Fakiolas

Peer review and feedback

Hans Niedderer 



\section{CONTENTS}

\section{A: TEACHERS' GUIDE}

1. Introduction to the Module

2. Connection of this module to other modules in Materials Science project

3. Background Information (for Teachers)

3.1. Sound and Noise

3.2. Sound Propagation

3.3. Sound Energy

3.4. Interaction between Sound and Matter

3.4.1. Sound Transmission

3.4.2. Sound Absorption

3.4.3. Interaction at the Interface between Two Mediums

3.5. Sound Attenuation through a Solid Medium

3.5.1. Sound Attenuation by Reflection

3.5.2. Sound Attenuation by Absorption

3.6. Sound Reflectors

3.7. Sound Absorbers

4. Prior Knowledge of Students

5. Aims of the Module

5.1. Scientific Concepts

5.2. Methodological / Procedural Skills / Competences

5.3. Reasoning Strategies

5.4. Attitudes

6. Pedagogical approach and context

7. Relevant ICT tools

8. Common Student Difficulties Involving Sound Phenomena

9. Monitoring Student Learning 23

10. Other Useful Information 24

11. References 
Unit 1: Sound wave - material interaction 35

$\begin{array}{ll}\text { Sequence of contents } & 35\end{array}$

Learning Targets $\quad 35$

1.1. Acoustic Problems of a Disco 36

Discussion of the Experiments and Exercises 36

$\begin{array}{ll}\text { Equipment } & 36\end{array}$

1.2. Why Can Sound Reach Any Corner of the Dance Floor? 36

Discussion of the Experiments and Exercises $\quad 36$

$\begin{array}{ll}\text { Equipment } & 37\end{array}$

1.3. How Can We Manage to Avoid Hearing Too Much Sound Outside

$\begin{array}{ll}\text { the Disco? } & 37\end{array}$

$\begin{array}{ll}\text { Discussion of the Experiments and Exercises } & 37\end{array}$

$\begin{array}{ll}\text { Equipment } & 38\end{array}$

Unit 2: Properties and Internal Structure of Sound Reflectors and Sound Absorbers 39

Sequence of Contents 39

Learning Targets 39

2.1. Which Characteristics Does a Good Sound Reflector Have? And a

Good Sound Absorber? 39

Discussion of the Experiments and Exercises $\quad 39$

Equipment 40

2.2. How Can We Explain That the Properties of a Material Affect its

$\begin{array}{ll}\text { Acoustic Behaviour? } & 41\end{array}$

Discussion of the Experiments and Exercises $\quad 41$

$\begin{array}{ll}\text { Equipment } & 41\end{array}$

Unit 3: Acoustic Conditioning and Soundproofing 41

Sequence of Contents 41

Learning Targets $\quad 41$

3.1. Comparing Materials. Which One Could Be Used to Soundproof? 42

Discussion of the Experiments and Exercises 42

$\begin{array}{ll}\text { Equipment } & 42\end{array}$ 


\section{D: DESCRIPTION OF MODULE DESIGN, DEVELOPMENT AND VALIDATION PROCESS}

ANNEX 3: Class observation grid 

A: TEACHER'S GUIDE 


\section{INTRODUCTION TO THE MODULE}

The Material Science (MS) Project, "University-school partnerships for the design and implementation of research-based ICT-enhanced modules on Material Properties" funded by the EU (Contract Number: SAS6-CT-2006-042942; 2007 - 09) aims to create a mechanism for integrating the results of science education research in school teaching practice for 1016 year-old students in the domain of Properties of Materials. In the framework of this project, a partnership between experienced secondary school science teachers and science education researchers from the Autonomous University of Barcelona (UAB) was established to design and develop a teaching/learning sequence (TLS) entitled "Acoustic Properties of Materials." The following sequence has been designed with this perspective and context in mind.

The partnership between teachers and researchers was proposed. As some studies of curriculum reform processes have shown (Pintó 2005), a passive role for teachers in the planning and designing phases can have deep implications in the implementation, often distorting the rationale of the innovation in a critical way. In such cases, teachers lack the necessary sense of ownership as they are not emotionally involved in the innovation (Andersson and Bach, 2005, Ogborn, 2002) and also they have not had the opportunity to learn enough about it, thus lacking the necessary knowledge. In this sense, the Spanish module for the Materials Science project suggests strong universityschool collaboration for the development of the sequences. Taking advantage of knowledge and experience of the two groups of contributors can bridge the gap between research and practice while improving the quality of science education offered to the students.

\section{The Rationale of the Module}

Materials Science is a field that investigates the relationship between the structure of materials and their properties, their performance and the way of characterising and processing them (see figure 1, Materials Science Tetrahedron). The final objective of this field is to understand the materials in a

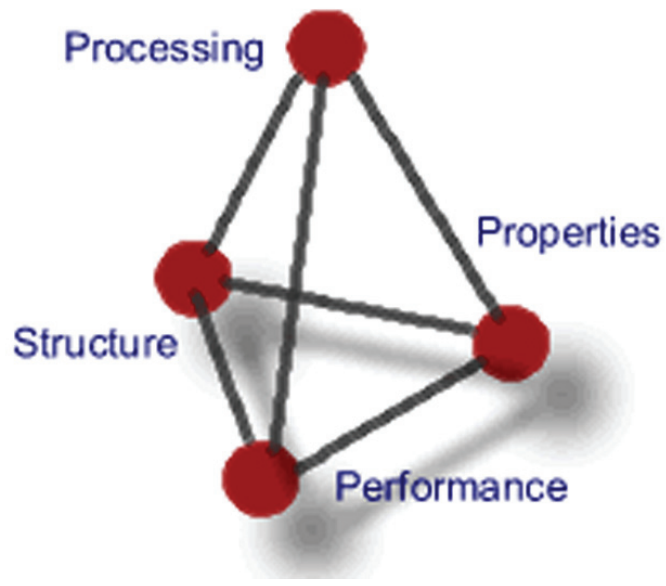

FIGURE 1. MATERIALS SCIENCE TETRAHEDRON

fundamental way so that we can design and create new materials with the desired properties.

Following this perspective, our module on the acoustic properties of materials is focused on analyzing the relationships between acoustic properties of some materials and their fundamental structure in order to account for their acoustic behaviour, that is, the way materials behave in front of sound regarding attenuation. Therefore, the module has an orientation as a subject of Materials Science.

Materials Science could be considered as a meeting point between different disciplines such as physics, chemistry, engineering, electronics, etc. We agree with the expert polymer scientist Michael Rubner at the Massachusetts Institute of Technology when he says: "It's time to stop looking at science just as chemistry, physics or biology. We need to recognize material science as fundamental".

Materials Science facilitates the relationship between theory and observations and the characterization of physical objects. This approach allows a mix of both the practical and theoretical nature of the subject.

The approach to the study of Sound and Acoustics in secondary school probably provides an innovative orientation that could make teachers more engaged in such a challenge. 
Relevance of the module in terms of developing an awareness of the role of Science in social contexts

There is a growing sensitivity in many societies to not disturb other people and not pollute. For instance, everybody tries to avoid spreading their contagious infections. It is also the case that people have become more sensitive to the fact that second hand cigarette smoke is very harmful. In summary, across different situations we realise that nowadays people are more aware of their environment and avoid harming those around them by trying to respect individual decisions and needs.

However, this sensitivity does not always exist in relation to sound. It is very common to not be able to understand each other in restaurants, even more so in discos and other public places with lots of people. In buses, subways or trains, we can hear conversations because people talk loudly with others in the same space or by mobile phone. At home, it is common to hear noises from neighbours. The problem is even worse if we consider noise in the streets regarding traffic in rush hour, when horns are used almost without limits.

In short, the human ear is frequently "assaulted" by all sorts of noise and sounds produced around us, and it seems that not many cautionary measures are taken in order to avoid them. As there is little sensitivity to this problem, there are few laws which state regulations, except perhaps for the most serious cases. Important building solutions for the soundproofing of buildings are hardly established either. New citizens should be educated with sensitivity to this problem.

Education on noise pollution needs to be accompanied by a real understanding of how sound propagates and how we can reduce sound intensity levels. "Acoustic properties of materials" is a teaching and learning sequence aimed at contributing to the education on acoustics. It is focused on the subject of sound attenuation with appropriate materials, introducing the concept of sound absorption and sound reflection, in an adequate level of depth for 14-16 year-old students from the comprehensive school. The sequence is intended to evidence the need for soundproofing a disco so that each of its spaces has the appropriate sound intensity level and also that sound becomes attenuated as much as possible outside.

\section{CONNECTION OF THIS MODULE TO OTHER MODULES IN MATERIALS SCIENCE PROJECT}

The sequence promotes that students are engaged in analyzing how different materials, each of them with their own properties, behave differently in relation to sound. This approach to the study of a topic on Physics (sound and sound attenuation), emphasizing the properties and internal structure of materials and their behaviour in different kinds of physical interactions, is the main connection element with other modules in Materials Science project. The field of Materials Science is not usually dealt with in school curriculums, so the approach of the teaching/learning sequence supposes an innovation.

This sequence is closely related to other ones designed for the common European project not only because they all deal with Materials Science, but also because the pedagogical and methodological approaches are shared among the partners. Some examples of the common features in the design of the modules are:

- The design of the materials has been done in a partnership between science education researchers and secondary school teachers.

- The use of ICT is common to different modules. Particularly, the module on Acoustics shares the use of sensors for capturing data during the labwork with Electromagnetism (EM) module developed by the Cyprus Local Working Group (LWG).

- The pedagogical approach selected as a principle design of the module on Acoustics shares many features with different partners' modules.

Moreover, there are some connections regarding the contents:

- The module on Acoustics and the module on Optics (developed by the group from Naples) analyse different characteristics of the behaviour of waves. For instance, both modules deal with the concept of wave reflection, frequency, wavelength, etc. 
- Density is the central property addressed in the module developed by the Florina's group and it is also considered a relevant property to account for the acoustic behaviour of some materials.

Therefore, all the modules have a common base; they are innovative programs aimed at improving the quality of science teaching and learning in schools. We believe that the potential interest of these modules lies in the fact that:

- They are interdisciplinary. The modules link chemical and physical properties of materials with physics, engineering of material design, architecture, etc, which makes it an excellent topic for an integrated science teaching approach.

- They are related to everyday topics involving citizenry education classes, which are quite often controversial for young people.

- They promote the use of different ICTs generally available in schools for the teaching and learning of the different topics involved.

\section{BACKGROUND INFORMATION (FOR TEACHERS)}

The sequence is focused on the acoustic properties of materials. For this reason, some background information that is not introduced in this teaching sequence (but is required in order to understand it) is the content related to the nature of sound, propagation of sound and characteristics of sound waves. Starting from that point, this sequence deals with the phenomena related to the interaction of sound waves with solid materials (reflection, reverberation, attenuation, absorption), but also deals with the properties (density, porosity and elasticity) of sound absorbers and reflectors. All the contents that are part of the background information of this sequence are described below:

\subsection{SOUND AND NOISE}

Sound is defined as a perturbation (variations of pressure) of an elastic medium (air, or any other gas, liquid or solid) which propagates through waves. Sound can be characterized by the general properties of waves: frequency, wavelength, period, amplitude, velocity and direction (velocity vector or wave vector).

Generally speaking, noise is considered a sound with a loud volume. However, noise is defined as a sound which has random frequencies combined in random quantities. These frequencies are not harmonics of a fundamental frequency. This means that they do not have a particular relationship with each other. Noise does not produce a particular pitch or quality sensations.

\subsection{SOUND PROPAGATION}

We can represent the medium through which sound propagates as made up of a high number of particles in balance arranged in a three dimensional lattice. There are attractive and repulsive forces between these particles. This system can be depicted as a series of balls connected by elastic springs (ball-andspring representation).

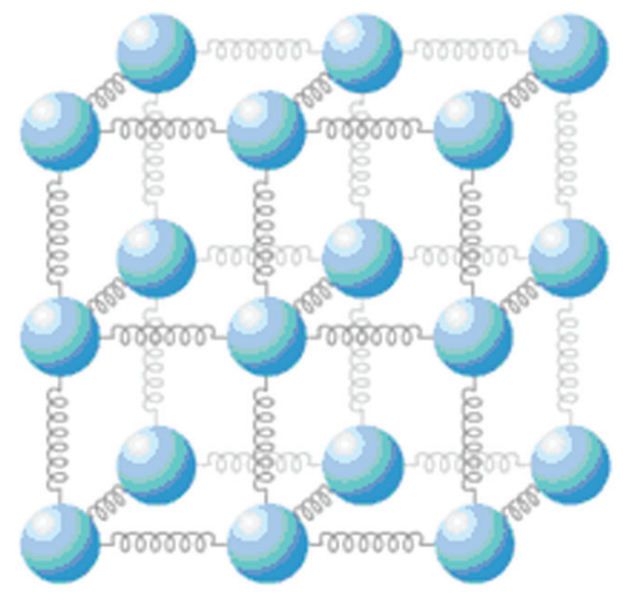

FIGURE 2. BALL-AND-SPRING REPRESENTATION

When one of these particles starts vibrating, the neighbouring particles do the same due to elastic reasons, although with a certain delay. The propagation of the initial vibration through the medium follows a wave motion, and in our case, a sound wave. Therefore, an elastic medium (solid, liquid or gas) is needed so that the sound waves can propagate.

\subsection{SOUND ENERGY}

In order to make any point of a medium vibrate, we need to provide energy to the medium. The propagation of sound waves implies a transfer of energy but does not imply a transfer of matter. Nevertheless, the particles of a medium oscillate around its equilibrium position but do not travel.

The sound produced by a source propagates in a spherical way. As sound waves move away from the 
sound source, the energy is distributed along an area that grows larger and larger. Therefore, the energy that is transmitted through a unit of area per second (intensity) is less and less. This decrease of energy per unit of area does not take into account the absorption produced in the medium; it is purely due to its geometrical distribution. This effect is expressed through the "inverse law of the square distance", which states that the intensity of a wave that propagates from a source in all directions decreases according to the square distance to the source.

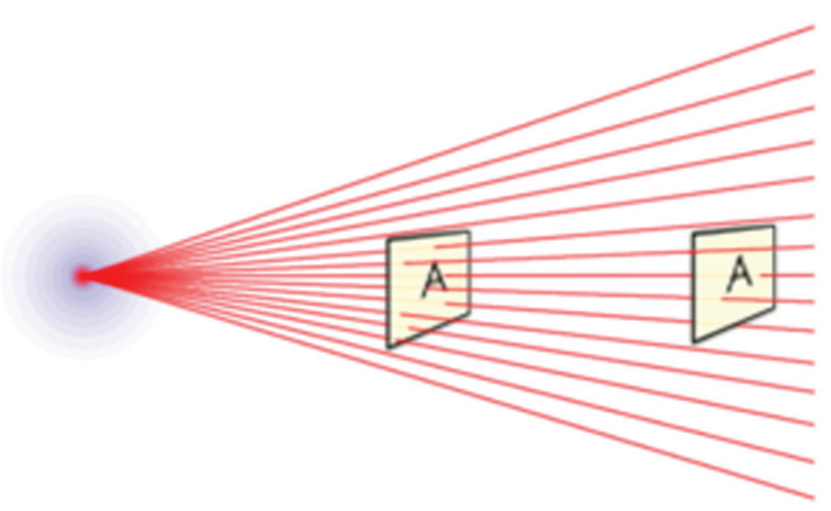

FIGURE 3. ILLUSTRATION OF THE "INVERSE LAW OF THE SQUARE DISTANCE”

\subsection{INTERACTION BETWEEN SOUND AND MATTER}

When sound waves which propagate through a medium reach a material, they can be reflected, can go through the material or they can dissipate inside the material. Therefore, when sound interacts with a material, it is partly reflected, partly transmitted and partly absorbed, respectively. Therefore, we can say that the energy of the incident wave is distributed into these three fractions.

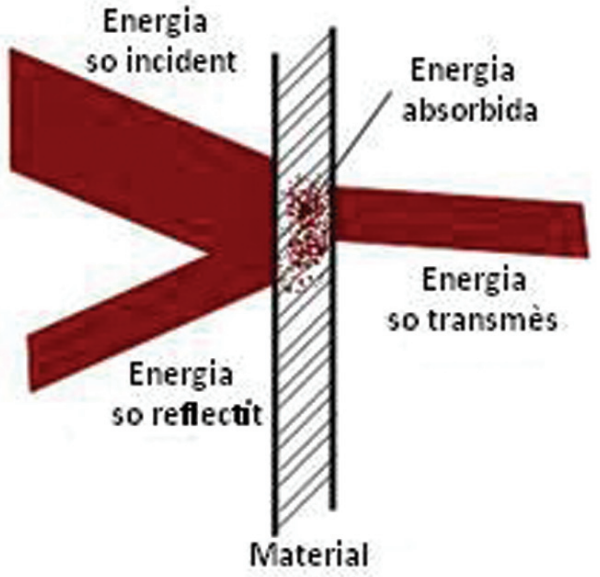

FIGURE 4. REPRESENTATION OF THE DISTRIBUTION OF SOUND ENERGY WHEN REACHES A MATERIAL
Generally speaking, part of the sound is reflected whenever there is a discontinuity or change in the medium through which sound propagates. Experimentally, it can be tested that the highest intensity level of the reflected sound wave is found in a direction that forms the same angle with the wall as that which is formed by the incident wave.

\subsubsection{SOUND TRANSMISSION}

Air is the most common medium that transmits sound to humans. However, when we hear a sound coming from the opposite side of a wall, sound has reached us through the air, but it had previously gone through the solid medium (the wall) before reaching our ears.

\subsubsection{SOUND ABSORPTION}

Sound waves stop propagating when their energy is completely dissipated within the medium. We say that sound has been absorbed or that sound energy has been dissipated within the medium. In the case of sound propagation through solid mediums, the vibration of the particles that form the materials contributes to sound absorption. Sound energy allows vibration and sound energy to dissipate inside it.

Sound energy dissipation can happen when airborne sound waves reach a solid material. In this case, sound can be absorbed because part of its energy is transferred to the other medium.

\subsubsection{INTERACTION AT THE INTERFACE BETWEEN TWO MEDIUMS}

When airborne sound waves reach the interface between two mediums (e.g. air and another material), part of the energy associated to sound is reflected and part of this energy is transferred to the material. From this part of sound which is transferred to the material, part will be absorbed within the material and part will be transmitted through the material.

\subsection{SOUND ATTENUATION THROUGH A SOLID MEDIUM}

Sound attenuation is due to absorption (dissipation of sound energy within the material) and also due to reflection (in the interface between mediums).

According to the principle of energy conservation, in order to transmit through a medium as less quantity of sound energy as possible, most of sound energy needs to be reflected or absorbed. When there is little 
sound transmitted through a medium, we can say that sound has been greatly attenuated.

Therefore, when we try to attenuate a certain noise, two different materials can be used: materials that reflect sound or materials that absorb sound (i.e., sound reflectors or sound absorbers, respectively).

\subsubsection{SOUND ATTENUATION BY REFLECTION}

The proportion of energy of an incident sound wave that penetrates a medium or is reflected when it reaches an interface between two mediums depends on:

a) The angle of incidence in the interface between both mediums. There will be more or less proportion of reflected energy depending on how the surface of separation between the mediums is (smooth, irregular, rough).

b) The properties of the materials through which sound waves propagate. There are materials that prevent the propagation of sound through them more than others. Density and elasticity of a material crucially affect its behaviour regarding sound attenuation. How can we interpret that these properties are so relevant to the proportion of sound energy that is reflected or penetrates a medium?

- Regarding elasticity, materials that are very elastic (higher bulk modulus) are less prone to deformation and require a higher force. Therefore, they reflect a greater part of the incident energy. They are not good absorbers but good reflectors.

- As for density, the greater mass per unit of volume of the material, the greater the inertia, and the greater the force is needed for causing a certain deformation of the material. Therefore, denser materials reflect more sound waves. This means they are good sound reflectors.

\subsubsection{SOUND ATTENUATION BY ABSORPTION}

Regarding sound absorption, the quantity of sound energy that is absorbed within a material depends on the following variables:

a) Structure of the material (porosity ${ }^{1}$ )

b) Frequency of sound waves

c) Thickness of the material object

Great absorption implies that most of the sound penetrates the material (little reflection in the interface). In this sense, sound absorbers have to be bad reflectors, and therefore, have to be less elastic and dense.

How can we interpret that these properties are so relevant to the absorption of sound energy within a medium?

Elasticity and density are not only related to sound reflection (the less elasticity and density of the material, the less sound reflection), but they are also directly related to the quantity of sound energy that is absorbed (dissipated) within the material. The more elastic the material is the higher force is needed in order to deform it. On the other hand, if the material is less elastic, the deformation reached applying the same force is higher. Part of the energy of the incident wave is dissipated due to these deformations. This is to say that most of the sound is absorbed within the material. In the same way, the denser the material is, the more force is needed in order to make its particles vibrate. Therefore, the particles that form the materials that are less dense can vibrate more, and consequently dissipate more energy.

\section{a) Structure of the Material}

When sound waves enter a porous material, their energy quickly decreases. This effect is due to the friction of the air inside the material (in the pores, between fibres) with the rigid walls or the skeleton of the material. Due to this friction, part of the sound energy is transferred to the solid skeleton of the material, by making its particles vibrate and therefore, by increasing its temperature (heating of the material). Moreover, when the sound goes through a porous material, sound waves are forced to change their direction frequently while they are propagating within the material, because there are several changes of medium or air-solid-air interfaces.

Porous materials with the same porosity but with a rigid skeleton are more absorbent than porous materials with a flexible skeleton. This effect happens because there is more relative motion and friction between air and a rigid material, and therefore, more

1. Porosity is the proportion of empty spaces in the total volume of a material and it is usually expressed as a percentage. That is, it is measured by calculating the quotient between the volume of empty spaces and the total volume of a sample and multiplying it per one hundred. 
energy is absorbed. Size of the pores also affects the capacity of absorption of a certain material (e.g. if the pores are too big the material attenuates sound very little).

\section{b) Sound Wave Frequency}

Regarding the frequency of sound waves, it has been empirically tested that the capacity of absorption of a porous material increases as the frequency of the sound wave increases. When the wavelength is short, and therefore, the frequency is high, particles of the medium vibrate more times per second. Thus, they dissipate more energy when propagating in any medium. For this reason, sound with high frequencies is more easily attenuated than low frequencies.

\section{c) Thickness of Objects}

Regarding the thickness of objects, it has been empirically tested that absorption due to porosity increases as thicker the porous material is, until a certain limit. This limitation effect could be explained taking into account that the fraction of the incident wave that has short wavelengths (high frequencies), which is the fraction that has an effect in porous materials, has already been absorbed through a certain thickness of the material while the rest of frequencies cannot be absorbed through the material. Therefore, the thickness of the material is not the most important factor to achieve a high absorption coefficient depending on the frequency. In any case, we are referring here to objects with a certain property -the thickness- but not a material property. Depending on the piece of material that we take, it will have more or less thickness, mass, volume, etc. since all of these are extensive properties.

\subsection{SOUND REFLECTORS}

Sound reflectors avoid sound propagation and reflect most of the energy associated to sound waves. These materials have the following properties:

- High elasticity

- High density

- No porosity

\subsection{SOUND ABSORBERS}

Sound absorbers reduce the fraction of the incident wave that reflects in the interface between two mediums. That is, they allow a greater amount of energy to penetrate the material. The absorber materials attenuate the energy of the sound wave inside the pores or due to deformations of the material. These materials have the following properties:

- Low elasticity

- Low density

- Porosity

\section{PRIOR KNOWLEDGE OF STUDENTS}

The sequence is aimed at 14-16 year-old students from compulsory secondary school. Therefore, this sequence is intended for students with a wide range of skills and rhythms of learning.

As the contents of this sequence are related to the topic of sound, preliminary ideas about sound (nature of sound, propagation of sound, characteristics of sound, etc) should be known before starting the module. Other basic concepts that are necessary as a pre-requisite for this sequence are related to properties of materials (density, elasticity, porosity). The particular model of matter is also essential in accounting for the relationship between the internal structure of materials and acoustic behaviour.

Therefore, the sequence requires that students have some previous knowledge of the following:

- Clear conceptual understanding of the nature of sound: how it originates and propagates through a medium.

- Distinction between different characteristics of sound: frequency, velocity of propagation, wavelength, amplitude, energy, intensity.

- Mastering mathematical formulas is not a prerequisite for this sequence, but students should be familiar with the interpretation of graphs.

Preliminary pages of the module are addressed to revise these concepts. 


\section{AIMS OF THE MODULE}

Throughout the sequence, students are supposed to understand some concepts and build some conceptual models that allow them to explain and predict different phenomena, and allow them to increase their understanding about the nature of science and its expected social role in the process.

The sequence "Acoustic properties of materials" has the following components:

\subsection{SCIENTIFIC CONCEPTS}

The designed teaching / learning sequence on "Acoustic properties of materials" has been structured as an enchainment of purposeful activities addressed to scaffold the process of modelling the role of sound absorbers and reflectors across three conceptual models:

- a model of sound attenuation based on energy terms, to predict and explain how the sound is distributed in the space: the energy associated to the sound wave is distributed when the sound reaches any object.

- a model of sound reflector materials and sound absorber materials in terms of their physical properties. This model allows to predict and explain how sound is attenuated (by reflection or absorption) when it reaches a material according to its physical properties. We focus on the relevant properties density, elasticity and porosity at the macroscopic level.

- a model of sound reflector materials and sound absorber materials in terms of their internal structure. The model allows us to predict and interpret how certain properties of a material influence its capacity of attenuating sound according to its microstructure.

Therefore, the previous conceptual models are gradually introduced: from explaining and justifying how materials behave regarding sound attenuation to explaining and justifying in terms of their physical properties (density, rigidity, porosity) and their internal structure.

The sequence has been structured in three main blocks. The first block deals with the phenomena associated to the interaction between sound and matter. Therefore, this block introduces the first conceptual model described above. The second block deals with properties and the internal structure of sound reflectors and sound absorbers. Thus, this second block introduces the second and third conceptual models. The third block was conceived to promote students' use or application of the previous conceptual models in a problem-solving situation. The scientific concepts that each block of the sequence addresses are:

1. Sound-material interaction. Distribution of energy as a model to explain the effects of interactions
a. Phenomena related to sound-material interaction: Reflection, Absorption, and Transmission.
b. Energy of the incident sound, reflected sound, transmitted sound, and absorbed energy.
c. Effects of the interaction sound-material: sound attenuation.

2. Sound absorber and sound reflector
a. Physical properties of sound absorbers and sound reflectors.
b. Internal structure of sound absorbers and sound reflectors
c. Mechanisms of sound attenuation of materials

3. Problem: noise pollution. Solutions: soundproofing and acoustic conditioning

\subsection{METHODOLOGICAL / PROCEDURAL SKILLS / COMPETENCES}

Throughout the sequence we intend to promote different kinds of competences. For this purpose, different kinds of activities and experimental work have been carefully designed to promote the development of the following competencies:

- Building conceptual models.

- Applying conceptual models and appropriate knowledge in a given situation to interpret and predict phenomena.

- Designing experiments with specific purposes.

- Using experimental data to interpret evidence and reaching conclusions.

- Reflecting on the implications of science and technological proposals or solutions to different problems. 


\subsection{REASONING STRATEGIES}

The module has been devised taking into account teaching strategies that can help to promote the ability of scientific reasoning. These teaching strategies have to do with:

- Starting from well-oriented intermediate steps (analytical approach). The Socratic dialogue tends to be a good approach to promote that ability.

- Raising global awareness problems to encourage students to design the procedures to find a solution (holistic approach).

The teaching approach is intended to be intellectually challenging in order to promote reasoning strategies, especially those concerned with modelling, structuring and the organization of ideas and inquiry skills (ex. making inferences, designing experiments, controlling variables, etc).

Modelling is best described in literature as:

1. Building or using models as entities (physical or visual/virtual objects) to help students to construct some concept or theory or to interpret phenomena. It is assumed that students will be acting in similar ways as scientists do, and so, facilitating the understanding of the nature of science.

2. Building conceptual models, understood as mental or cognitive representations of real-world processes or things that students build in their mind in order to explain / interpret phenomena (Glynn and Duit 1995). The perspective here is that conceptual models are coherent units of structured knowledge used to organize factual information into coherent wholes and to represent observable patterns of natural phenomena.

An important task for a scientist after having observed a phenomenon or process is to find an adequate explanation of it, either constructing a model or applying an existent one to justify what has been observed. Lesh and Doerr (2003) state that scientific models are conceptual systems consisting of elements, relations, operations and rules governing interactions that are expressed using some external representational system. Teachers introduce students to conceptual models in order to simplify natural phenomena, making the phenomena more understandable to students. Generally speaking, conceptual models are scientifically valid; they are consistent with scientists' understanding of the phenomena. When students learn science meaningfully in schools, they construct conceptual models consisting of related concepts, instead of lists of unrelated facts.

For example, students need to build a model of sound in order to conceptualize sound propagation. Depending on the level of the students, the model of sound propagation will be more or less sophisticated. That is, the conceptual model that students should / can construct will be more or less refined and close to the most advanced scientific models about a certain phenomenon.

In the sequence, sound propagation through a solid, for instance, is conceived as a disturbance of the internal structure resulting from an external perturbation that propagates due to "elastic springs" among the particles. Using this kind of mental representation, students can interpret all the phenomena around sound propagation through a solid (See part 3 "Background Information").

In both meanings of "modelling" it is assumed that modelling has the potential to scaffold the students' understanding of the processes of science when they are building, testing or revising their own models (being physical or conceptual ones). Research literature tells us that students develop the transferable skills of modelling when they are able to build or make use of conceptual models for exploring and predicting phenomena, constructing coherent meaning.

Both perspectives of modelling are not contradictory but refer to different aspects of the process of meaning-making and understanding science. In the first perspective, understanding science is perceived "as a complex set of modelling skills, that is, a set of cognitive skills for making and using models". In the second, understanding science means building conceptual models. The latter is a process of students' reasoning which is intended for the construction of models that should have the requirements of scientific models: (a) representing defined aspects of a phenomenon, being possible to be refined or falsified; (b) providing a mechanistic interpretation of the underpinnings of (certain aspects of) the phenomenon; (c) being useful to formulate predictions which can be put to the experimental test (Constantinou 2007). In the acoustics module, the second perspective of modelling is applied. 


\subsection{ATTITUDES}

We do not conceive teaching science as merely a process of building scientific knowledge (concepts, ideas, and theories) or scientific procedures. We understand that one of the aims is also to promote both (a) Scientific attitudes and (b) a positive attitude towards science.

(a) As Scientific attitudes we understand those dispositions typical of scientific work and essential to acquire certain inquiry skills. The sequence is intended to especially promote four attitudes:

- Curiosity, wondering and motivation for knowing more, asking questions about objects and phenomena, showing interest in the outcomes of experiments, using inquiry strategies.

- Creativity, originality, motivation for seeking individual solutions to different problems or situations, exhibiting original thinking in their interpretations.

- Critical thinking, in order to be able to justify their conclusions using evidence, pointing out contradictions in reports by their classmates and changing their ideas in response to evidence.

- Perseverance, regularity, in order to go on doing the activities beyond an initial enthusiasm, repeating an experiment in spite of apparent failure, completing an activity even though their classmates have finished earlier.

(b) A positive attitude towards science involves a particular way of seeing the scientific work of scientists and also the construction and evolution of scientific knowledge which encourages students to "go on". In this sequence, the selection of contents, the activities proposed and the teaching strategies planned are intended to make the learning of science become a motivating and stimulating activity. The context of this sequence shows the important role of science in providing a base for technological solutions that allow us to solve everyday problems. In this way, one of the aims is also to allow students to become interested in the complex adventure of science.

In this section, we could consider other more general attitudes, personal attitudes (interest in collaborating with classmates, appreciation for work quality, motivation for increasing personal autonomy, etc) or related to the work that will be developed in class or in the laboratory (awareness of the importance of organizing information, using computers and experimental equipments with accuracy, etc).

\section{PEDAGOGICAL APPROACH AND CONTEXT}

It is clear that the pedagogical approach of a module is closely related to the view that its designers have with regard to appropriate strategies for teaching and learning. In the case of the module on Acoustics, it was chosen to establish a partnership between teachers and researchers to design and develop the sequence. The collaborative work between the participants was intended to co-construct and share the rationale of the module (content and pedagogical approach), so that there was an "ownership" on the teacher's behalf (Ogborn, 2002). Although this objective involves engaging teachers and researchers in a long process, the results are promising.

The design of the module has taken into account some issues regarding the pedagogical approach that we could summarise:

\section{Contextualisation in Everyday life}

The sequence is guided from an initial everyday situation: a disco that should avoid the extremely high sounds that propagate to the neighbourhood. Noise is coming both through the air and through the wall. How is sound reaching the neighbours? Can it be stopped or reduced using earplugs? Are there other solutions?

\section{Interdisciplinary approach}

The innovative contents of the sequence about acoustic properties of some materials are related to the subject of Materials Science. This innovative approach to sound, starting from acoustic properties of materials, has provided a mix of practical and theoretical nature of the subject and also of the sequence. This approach of analysing Sound and Acoustics has provided an innovative orientation that challenges teachers and engages them with the module.

Sound is a classic area of Physics present in most science syllabuses. Linking sound with the important everyday idea of noise pollution is also common in syllabuses with an STS or contextualised approach. Our purpose was to give another dimension to the module: a technological approach to introduce ideas of 
materials science in the current curriculum. Within the Spanish syllabus, implementing a sequence on Materials Science means to link up classical Physics and Chemistry, since we will have to take some ideas about the structure of matter (subject analysed mainly from the Chemistry point of view in the Spanish present syllabus) in order to explain the propagation and attenuation of sound (subject analysed mainly from the Physics point of view).

\section{Technological approach}

The activities of the sequence have a technological approach. One of the main goals of education is to achieve both scientific and technological literacy. In this sense, an acoustic education is a relevant and interesting topic since it deals with both scientific and technological aspects in order to allow students to understand why and how sound can be attenuated using different materials.

\section{Incorporated Innovations}

A school topic as challenging as Materials Science, together with some pedagogical innovations, implies a lot of support for the participating teachers. The key innovations that have been incorporated are:

- Conceptualizing sound in the interaction with the structure of the materials and medium through which it is propagated. In part 3 "Background information", the scientific models and concepts that we expect students to build along the sequence are described. However, an adequate "didactical transposition" needed to be done according to the students' level. The "educational reconstruction" of the contents to be taught was the result of discussions and agreements among all the members collaborating within the partnership in the design of the sequence. Furthermore, an extensive analysis of different bibliographical resources (from materials science, acoustics, engineering, architecture, etc) was also necessary.

- Understanding Modelling as a useful process for helping students to build scientific knowledge and that allows them to transfer it to many situations. In part 5.3, we have already developed our views on modelling.

- Sharing the view of inquiry teaching approach and inquiry skills, considering the former a useful way of teaching in order to promote an inspiring view of scientific knowledge and, the later as scientific abilities. In order for students to understand inquiry to facilitate students' learning science and to develop inquiry skills, teachers need to be well-versed in inquiry-based methods. Since most teachers have not had opportunities to learn science through inquiry or to conduct scientific inquiries themselves inside or outside the classroom, we provided them with some ideas and hints about inquiry:

http://books.nap.edu/html/inquiry_addendum/ch2.html

We share the perspective proposed for the developers of the Guide for Teaching and Learning, as addendum to the National Science Education Standards on Scientific Inquiry, especially when they emphasise inquiry as a perspective for promoting curiosity and support for building models. They challenge teachers to:

- ask good questions.

- design effective investigations.

- carefully craft students explanations of what they found.

- focus on the "big ideas" in physics, not in a piecemeal manner, jumping from one topic to the next, giving students a broad vision of physics.

- organize small-group activities structured to encourage the team members to talk, debate, and come up with predictions based on initial observations and with explanations based on evidence.

- begin lessons with activities to set the context for helping students discuss conceptual ideas.

- take frequently behaviours as listening, clarifying statements, and open-ended questioning.

- "Tell me more about Y"

- "What is the evidence for that conclusion?"

- "How did you decide on that explanation over the one you were convinced of yesterday?"

- encourage the students to pose questions that arose in their minds instead of always framing the questions.

- increase the amount of time students are talking.

As the above site mentions, the essence of inquiry does not lie in any elaborate, equipment-intensive laboratory exercise. It lies, rather, in the interactions between the student and the materials, as well as in the teacher-student and student-student interactions that occur dozens of times in every class period.

In view of the methodology to prepare the module, and 
not intending to impose anything, the researchers' stance consists of taking care of the characteristics of the module in order for them to be accepted, shared and integrated in the sequence, as a result of an agreed work. Learning is a long process and the incorporation of innovations on the behalf of experienced teachers requires the ability to convince students with sound arguments and time.

During the research project STTIS, it was clearly evidenced that teachers transform intentions, goals, procedures of any activity that is felt far from their usual, accepted or incorporated ways of proceeding. However, when teachers are aware of the interest of a new approach, they are more prone to change the usual way of doing. Bearing this perspective in mind, the refinement of each of the activities should be agreed upon among all the partners even if more time is required to achieve these means.

In spite of this, the contents of the sequence and the sessions of theorising / reflecting on the teaching process will not decide the real pedagogical approach of the teaching that will be carried out. It is necessary to decide if the implementation easily fits the agreed patterns or if difficulties come up in the class practice.

The Approach of Some Activities

Some of the activities proposed to students consist of laboratory work where a learning cycle is proposed. Students work in pairs or in groups of 3-4 people.
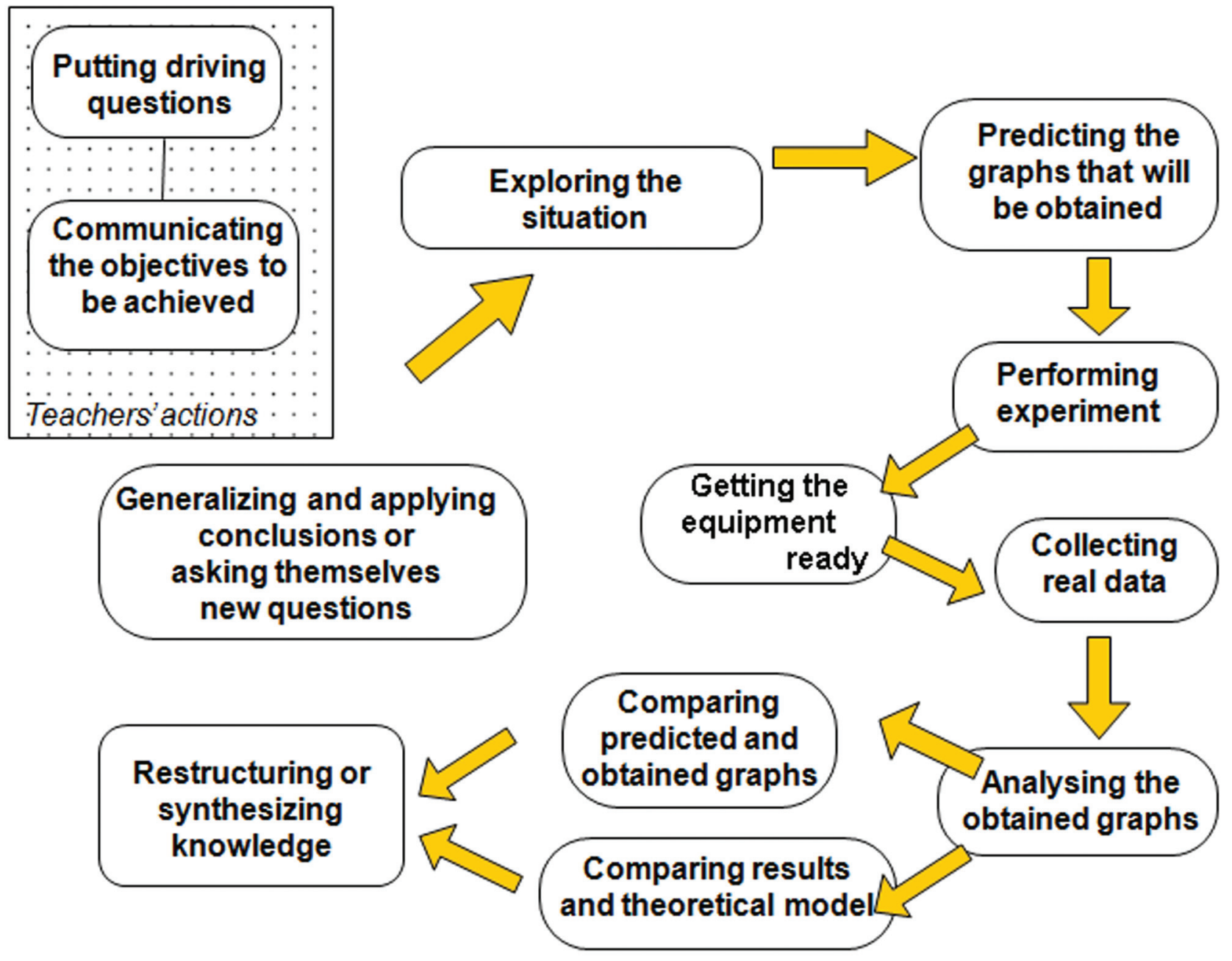

FIGURE 5. LEARNING CYCLE FOR LABWORK WITH ICT TOOLS 
Sample of activities already included in the sequence:

- Questions to be discussed amongst their fellow peers in class about the different behaviours of materials for the attenuation of sound, using an elementary model of particles. The discussion and application of the model should lead to ideas of an elastic property and an inertial property of the material having a role in sound attenuation.

- Exercises for synthesising different concepts and relations and organizing ideas through different sorts of activities.

- Prediction and justification of the acoustic behaviour of different materials according to a visual observation. Planning some experiment to test one's own predictions. Designing an experiment to analyse if certain pieces of different materials are good reflectors or absorbers.

- Inquiring about sound absorbers. Observations using magnifying glasses and microscopes of different pieces of sound absorbent materials. Inferences from the observations, keeping in mind or refining the model of sound propagation and the model of sound absorption in materials depending on its structure, porosity, density, etc.

- Using an instrument (sound level meter) to measure different levels of sound intensity (in decibels).

- Experimental testing of sound attenuation, using different sound absorbers and a sound level meter. Inquiry about the role of different properties of the materials: density, porosity, thickness, etc are relevant in their capacity to attenuate sound.

- Communicating verbally (oral and written) results of experiments or observations.

\section{RELEVANT ICT TOOLS}

Some ICT tools have been selected for enhancing learning in the sequence and for scaffolding the process of building students' conceptual models around sound attenuation. A brief description of the characteristics of these technologies that make them suitable for our purposes is provided below:

Simulations selected to allow support for the visual representations of the model of wave, sound propagation, through different mediums. Special importance is given to select proper simulations that have even been designed purposefully based on research results. For instance, the simulation called SimulaSON.

(http://icar.univ-

lyon2.fr/membres/jvince/simulaSON/telecharge.html) was developed by researchers from the University of Lyon, and was analyzed as a useful resource to teach nature and propagation of sound to secondary school students.

MBL technology as a set allowing for taking data in real-time is a good technology for analysing graphs of sound intensity levels. It allows us to measure sound attenuation using different materials. Students are proposed to be involved in inquiry tasks where they analyse the acoustic behaviour of different materials (with known properties) in front of different sounds (of different intensities). However, it is possible that in some cases the sound level meter is used without being connected to the computer for the same purpose.

In all the activities using ICTs, students will be the main actors during the inquiry activities since they have to play a very active role.

\section{COMMON STUDENT DIFFICULTIES INVOLVING SOUND PHENOMENA}

Identifying the preconceptions that prevent students from properly understanding sound phenomena deserves a central place within the science curriculum and should also be addressed in science education. There are some studies that have focused on students' representations and common preconceptions of sound, before or after a formal instruction.

\section{Students' conceptions on sound nature and sound propagation}

Linder (1992) examined the difficulties that undergraduate physics students had to understand sound. This research study revealed that the preconceptions about sound that students held were:

- Sound is an entity which is transported by individual molecules (sound particles), which move along a medium.

- Sound is an entity which is transferred from one molecule to the other through a medium. 
- Sound is a limited substance which travels with a certain impetus, and is generally represented as an air current.

- Sound is a substance which travels following a pattern.

- Sound is related to the concept of waves (in most cases, sound is not distinguished from light since wave equations are identical).

A later study of this author (1993) consisted of analyzing the explanations of the same students with the purpose of categorizing the different ways of talking and thinking about the topic. Linder highlighted three different conceptualizations on the factors that affect the velocity of sound propagation:

- Regarding the first conceptualization, molecules were presented as an obstruction to the propagation of sound through the medium.

- Regarding the second conceptualization, the distance between molecules, or distance that a molecule needs to travel before interacting with a neighbouring molecule, reflected the role of density as a factor that affects the velocity of sound propagation.

- Regarding the third conceptualization, elasticity and density were identified correctly as factors that affect sound velocity. Nevertheless, the students conceptualized elasticity without giving it a physical meaning. Elasticity is conceptualized in terms of compressibility, and is inversely proportional to density.

A research carried out by Saura and De Pro (1999) identified both the conceptual and procedural ideas about sound that students from vocational training had. These authors highlighted that the students hold a biased conception about waves and therefore, further clarification about their characteristic magnitudes (frequency, amplitude and wavelength). Furthermore, the study revealed that the students have difficulties appropriately conceptualising vacuum and matter composition, and hold misconceptions on energy, which could condition their learning of related concepts. Finally, this study also evidenced that students use a corpuscular model when reasoning about sound.

The findings of the research carried out by Hrepic, Zollman and Rebello (2002) indicate that students mainly use two fundamental models of sound propagation: the scientifically accepted wave model and the dominant alternative entity model. According to the latter, sound is an entity which is different from the medium where it propagates. The properties associated with sound within the entity model are:

- Sound is independent; sound propagates through the vacuum (it does not need medium).

- Sound is material; it is a substance and has mass.

- Sound passes through empty spaces between the medium particles (seeping).

- Sound is a propagation of sound particles that are different from medium particles.

These authors reached the conclusion that students' answers depended on their mental models and the context of the question they answered.

Mazens and Lautrey (2003) analyzed to what extent the knowledge on sound of primary school students was coherently organized. These authors tried to determine if the pupils applied properties of physical objects to sound, such as substantiality, weight and permanence, or if they considered sound as a vibratory process. Younger students considered sound as an object in greater proportion than older students. The students attributed the property of substantiality to sound more often than weight or permanence. Based on the idea of substantiality, the authors identified four mental models:

- Sound cannot go through other objects unless these have holes.

- Sound can go through solids if it is stronger or harder than the solids.

- Sound is not material.

- Sound is a vibratory process.

These authors concluded that the conceptual change with regard to sound does not happen by suddenly transferring the concept from the ontological category of matter to the category of process, but rather that this process is a slow and gradual one which consists of revising one's own beliefs.

The research carried out by Wittmann, Steinberg and Redish (2003) showed that students seemed to think about sound waves using a common-sense interpretation based on sound waves exerting a onedirectional force on the medium through which they travel. In summary, the students:

- map object-like properties onto sound waves, 
- treat them as solid and pushing through a medium, and

- do not correctly interpret the event-like properties that are more appropriate in this setting.

For this reason, most of the students had great difficulties distinguishing between the propagation of sound waves and motion of the particles of the medium through which sound waves travel.

The research carried out by Eshach and Schwartz (2006) showed that middle school students perceived sound with the following characteristics:

- Sound is containable in different spaces or mediums ("sound may be inside bubbles or may be locked in closed spaces").

- Sound is able to move or be moved.

- Sound is able to push and be pushed ("sound is pushed by air, by explosion, by water, by a barrier, by the ground, sound hits the walls and bounces back and is attracted by a stethoscope").

- Sound drag when moving in contact with a surface.

- Sound is consumable. ("Sound is not a stable substance, does not conserve”).

- Sound has a definite location, it is limited.

- Sound keeps its "identity", that is, it remains as a material which is different from the substance which it interacts with.

- Although some students use the apparently correct expression "sound waves", this term can be also interpreted literally, rather than conceptually, as if the term wave indicates the form of the "way of the voice".

- Sound propagation requires a force (conception of sound as a particle).

In short, these research studies evidenced that previous knowledge of students in any educational level tends to be materialistic or "based in substances", since students have understood and experienced the behaviour of material objects and other substances throughout their life. This implies that students tend to attribute properties or behaviours of material substances even to physically abstract concepts.

\section{MONITORING STUDENT LEARNING}

We consider the assessment as an integral part of the learning and teaching cycle since it provides information for both teachers and students on the processes of and the improvement in learning and teaching. It is well known that the nature of assessment practice significantly affects what science is taught and hence, what science is learned. In fact, from our perspective, when referring to assessment we are talking at the same time about formative and summative assessment processes, having different purposes and results.

a) Formative assessment serves as a diagnostic tool to provide feedback to help improve students' learning. It can occur at any time and is used to influence teacher's plans to understand specific learning experiences and student needs. Black and William (1998a) emphasize the relevance of such assessment and also the self-assessment of students since it raises students' performances. They point out that "students should be trained in self-assessment, so that they can understand the main purposes of their learning and thus what they need to achieve." The self-regulation after assuming the objectives that the teacher has decided upon, has been evidenced as an important tool for better performances. Moreover, a continued assessment activity will promote further analysis of the sequence goals and the necessary adjustments or revisions.

b) Summative assessment typically occurs at the end of a learning activity to determine its impact on student learning. The results of such assessments are presented in a way that they can be summarized and compared with other evidence so that judgments can be made.

\section{Instruments}

A variety of strategies and instruments were adopted to assess student learning styles.

For the purpose of student assessment learning from the sequence "Acoustic Properties of Materials", we have developed some instruments for summative assessments:

- First, we developed a list of learning targets to be achieved, which were formulated to be assessable. 
Learning targets included an action verb and a statement of ability. For example, "Students should be able to analyze ..." or "Students should be able to compare ..." They will make possible that teachers make judgement on students' performance, and inform students of their progress and also can be used by students to monitor their own learning.

- Later, we developed methods or criteria for determining the degree to which students had achieved an identified learning target. These methods allowed us to collect information about: knowledge and understanding of scientific concepts, methodological / procedural skills, and reasoning abilities.

In all the cases, we assume that a scientific concept is learnt when it can be successfully transferred to different contexts.

Students' worksheets and class observations have been the instruments mostly selected for the formative assessment. The student worksheets provide students with a wide range of activities (e.g., expression of previous ideas through multiple-choice, open-ended or true/false questions, asking students to justify their selections or answers). These activities also allow teachers to explore students' preconceptions or reflections after a unit. The sequence also includes activities for self-assessment or peer-assessment.

Observations also need good guidelines with clear criteria in order to make judgements about students' discussions, explanations that allow teachers to provide an appropriate feedback. Probably openended discussions with the whole class will not be a matter of our assessment even though teachers will use them for detecting student progress.

Student motivation is measured with questionnaires before and after the implementation of the sequence. Furthermore, a representative sample of students is selected for semi-structured interviews at the completion of the sequence.

\section{OTHER USEFUL INFORMATION}

Relevant articles of research on students' conceptions and alternative ideas

Our selected bibliography includes students' conceptions on:

- Physics of sound (Linder 1987; Borstad 1989; Linder 1989; Watt 1990; Linder 1992; Linder 1993; Maurines 1993; Barman 1996; Magnusson 1996; Mazens 1997; Kelly 1998; Rosser 1998; Staver 1998; Kelly 1999; Saura and de Pro 1999; Leite 2001; Treagust 2001; Vince 2001; Mazens and Lautrey 2003; Wittmann 2003; Eshach and Schwartz 2006).

- Concept of wave that are important in sound teaching and learning (Segarra 1993; Wittmann 1998; Wittmann 1999; Benhassoun 2001; Sarantopoulos 2001; Utges 2001; Wittmann 2002; Wittmann 2003).

- Structure of matter (Scott 1992; Keig 1993; Pujol 1993).

- Particulate nature of matter (Hibbard 1975; Novick 1978; Novick 1981; Comber 1983; Driver 1983; Brook 1984; Wightman 1986; Gabel 1987; Johnston 1990; Meheut 1990; Pritchard 1990; Hwang 1993; Williamson 1995; de Vos 1996; Lichtfeldt 1996; Rohr 1997; Kokkotas 1998; Lee 1999; Rohr 1999; Sanger 2000; Selley 2000; MaNaim 2001; Selley 2001; Bunce 2002; Harrison and Treagust 2002; Singer, Tal et al. 2003; Snir, Smith et al. 2003; Velázquez-Marcano, Williamson et al. 2004; Williamson, Huffman et al. 2004; Sanger 2005; Jiménez Gómez, Benarroch et al. 2006).

- Physical properties of matter (Klopfer 1992).

- Atoms and molecules (Buck 1990; Voorde 1990; Griffiths 1992; Harrison 1996; Harrison 1996; Harrison 2000).

- Chemical bonds (Boo 1998; Harrison 2000).

- New materials (Kruger 1989; Kruger 1992; Aho 1993; Calvelli 1996; Stavy 1996; Schibeci 2000; Papalexopoulos 2001; Krnel and Glazar 2003). 
Web sites of teaching proposals and projects for teaching Materials Science

High school

MATERIALS SCIENCE AND TECHNOLOGY TEACHERS' WORKSHOP (MAST)

http://matse1.mse.uiuc.edu/

MATTER PROJECT (Computer Based Learning Resources in Materials Science done by Materials Science Researchers in UK.

http://www.matter.org.uk/default.htm

For schools: http://www. matter.org.uk/schools.htm

site: TEACHINGIDEAS, section MATERIALS AND

THEIR PROPERTIES (list of basically hands-on activities for primary school teaching)

http://www.teachingideas.co.uk/science/contents2ma terials.htm

site: SCIENCEBUDDIES, section PROJECT IDEAS ABOUT MATERIALS SCIENCE (different ideas for school research projects with different levels of complexity)

http://www.sciencebuddies.org/mentoring/project_ide as/home_MatlSci.shtml

University

PRINCETON CENTRE OF COMPLEX MATERIAL, outreach programmes for teachers and undergraduates

http://www.princeton.edu/ pccm/outreach/index.htm

COMPOMENT FAILURE MUSEUM, Open University. (includes examples of problems of some materials as the starting point for analysing their properties)

http://technology.open.ac.uk/materials/mem/

MATTER PROJECT, lead by University of Liverpool (Computer Based Learning Resources in Materials Science done by Materials Science Researchers in UK) http://www.matter.org.uk/default.htm

For universities:

http://www.matter.org.uk/universities.htm

MATERIALS INTERACTIVE WEBSITE from the National Physical Laboratory (a set of applets in materials Science)

http://materials.npl.co.uk/netshare/guest/

MATWEB (database of properties of materials)

http://www.matweb.com/index.asp?ckck=1
UK CENTER FOR MATERIALS EDUCATION (includes a database of educational resources about Materials Science teaching)

http://www.materials.ac.uk/index.asp

Other interesting links

TOP 50 moments in the history of materials (by the TMS: The Minerals, Metals and Materials Society)

http://www.materialmoments.org/vote.html

\section{Web sites of teaching proposals for teaching sound/ waves \\ Secondary school \\ Sound hygiene, from NONOISE ORGANISATION \\ http://www.nonoise.org/ \\ http://www.nonoise.org/resource/educat/soundhyg/s oundhyg.htm}

MUSIC INVENTIONS, unit about sound and developing musical instruments

http://www.op97.k12.il.us/instruct/thematic/musicinvent.html

High school

THE PHYSICS CLASSROOM (tutorial on sound and waves)

http://www.glenbrook.k12.il.us/gbssci/phys/Class/sou nd/soundtoc.html

SALFORD ACOUSTICS, SOUND AND VIDEO, University of Salford (students' resources for school and college)

http://www.acoustics.salford.ac.uk/schools/index.htm CONCEPT MAPS FOR TEACHING ACOUSTICS (proceedings of the 2ond international conference on concept mapping)

http://cmc.inmc.us/cmc2006Papers/cmc2006-p36.pdf SCIENCE OF SOUND IN THE SEA, University of Rhode Island

http://www.dosits.org/science/intro.htm

University

INSTITUTE OF SOUND AND VIBRATION RESEARCH (teaching materials on waves and acoustics)

http://www.isvr.soton.ac.uk/SPCG/Tutorial/Tutorial/Sta rtCD.htm 
NATIONAL PHYSICAL LABORATORY (resources on acoustics)

http://www.npl.co.uk/acoustics/

WORLD OF MATERIALS ESSAYS from the MATERIALS RESEARCH SOCIETY (MRS) educational resources

http://www.mrs.org/s_mrs/sec_subscribe.asp?CID=2 725\&DID=171681\&action=detail

\section{SOUND AND VIBRATION ANIMATIONS}

http://www.gmi.edu/\%7Edrussell/Demos.html

(Dr. Dan Russell)

http://www.acs.psu.edu/users/sparrow/animations.html

(Victor W. Sparrow)

\section{Simulations}

Interactive Simulations from the PhET Project

(University of Colorado)

http://phet.colorado.edu/new/simulations/sims.php?si

$\mathrm{m}=$ Sound

\section{NDT Resource Center}

http://www. ndt-ed.org/EducationResources/HighSchool/Sound/hs_sound_index.htm

Learning resource for GCSE / Key Stage 4 Science of the University of Salford

http://www.acoustics.salford.ac.uk/schools/index1.htm

Project of soundproofing of the University of Vigo

http://webs.uvigo.es/gcastro/PFC/PROYECTO_ZALO _archivos/Aislamiento_ampliado.swf

http://webs.uvigo.es/gcastro/PFC/PROYECTO_ZALO _archivos/Ejemplos_aislamiento.swf

SimulaSON designed by researchers of the University of Lyon

http://icar.univ-lyon2.fr/membres/jvince/simulaSON.zip

\section{REFERENCES}

1. Aho, L., Huopio, J., Huttunen, S. (1993). "Learning science by practical work in Finnish primary schools using materials familiar from the environment: A pilot study". International Journal of Science Education 15(5): 497-507.

2. Andersson, B. \& Bach, F. (2005). On designing and evaluating teaching sequences taking geometrical optics as an example. Science Education, 89 (2), 196-218.

3. Barman, C., Barman, N., Miller, J. (1996). "Two teaching methods and students' understanding of sound". School Science and Mathematics 96(2): 63-67.

4. Benhassoun, M. S., Grea, M. J., Maurines, L. (2001). Elements to allow teaching wave phenomenon in relation with the teacher knowledge and practical skills. Proceedings of the Third International Conference on Science Education Research in the Knowledge Based Society, Vol. 1. D. Psillos, Kariotoglou, P., Tselfes, V., Bisdikian, G., Fassoulopoulos, G., Hatzikraniotis, E., Kallery, M. Thessaloniki, Greece, Aristotle University of Thessaloniki: 297-300.

5. Black, P., Wiliam, D. (1998). Assessment and classroom learning. Assessment in Education 5(1): 7-74.

6. Boo, H. (1998). "Students' understandings of chemical bonds and the energetics of chemical reactions". Journal of Research in Science Teaching 35(5): 569-581.

7. Borstad, J. L. (1989). "But I've been pouring sounds all day". Paper presented at the annual meeting of the Canadian Society for the Study of Education (CSSE), Quebec City: 1-16.

8. Brook A., Briggs, H., Driver, R. (1984). Aspects of secondary students' understanding of the particulate nature of matter. Leeds, University Leeds, Centre for Studies in Science and Mathematics Education.

9. Buck, P. (1990). Jumping to the atoms: The introduction of atoms via nesting systems. Relating macroscopic phenomena to microscopic particles: a central problem in secondary Science Education. P. L. Lijnse, Licht, P., Vos, W. de, Waarlo, A. J. Utrecht, CD-B Press: 212-219. 
10. Bunce, D. M., Gabel, D. (2002). "Differential effects on the achievement of males and females of teaching the particulate nature of chemistry". Journal of Research in Science Teaching 39(10): 911-927.

11. Calvelli, G., Capitanio, M., Di Blasi Burzotta, R., Furlan, D., Merlo, A. (1996). Teaching the changes of state of matter in the primary school. Teaching the science of condensed matter and new materials. M. Michelini, Jona, S., Cobai, D. Udine, Forum: 429-433.

12. Comber, M. (1983). "Concept development in relation to the particulate theory of matter in the middle school". Research in Science and Technological Education 1(1): 27-39.

13. Constantinou, C. (2007). Modeling in Science and Education. Proceedings of the Sixth International ESERA Conference. Malmö, Sweden.

14. De Vos, W., Verdonk, A. (1996). "The particulate nature of matter in science education and in science". Journal of Research in Science Teaching 33(6): 657-664.

15. Driver, R. (1983). "An approach to documenting the understanding of 15 years old British children about the particulate theory of matter". Research on Physics Education. Proceedings of the first international workshop. La Londe les Maures: 339-346.

16. Eshach, H. and J. L. Schwartz (2006). "Sound stuff? Naive materialism in middle-school students' conceptions of sound". International Journal of Science Education 28(7): 733-764.

17. Gabel, D. L., Samuel, K. V., Hunn, D. (1987). "Understanding the particulate nature of matter". Journal of Chemical Education 64(8): 695-697.

18. Glynn, S., Duit, R. (1995). Learning Science Meaningfully: Constructing Conceptual Models. In S. Glynn \& R. Duit (Eds.), Learning Science in the Schools: Research Reforming Practice. Lawrence Erlbaum Associates, Publishers. New Jersey: 133.

19. Griffiths, A. K., Preston, K. R. (1992). "Grade-12 students' misconceptions relating to fundamental characteristics of atoms and molecules". Journal of Research in Science Teaching 29(6): 611-628.
20. Harrison, A. (1996). "Learning about atoms and molecules: Using an ontological perspective to enhance our understanding of conceptual change". Paper presented at the annual meeting of AERA, New York, April 1996: 1-15.

21. Harrison, A., Treagust, D. (1996). "Secondary students' mental models of atoms and molecules: Implications for teaching chemistry". Science Education 80(5): 509-534.

22. Harrison, A. G. and D. F. Treagust (2002). The particulate nature of matter: Challenges in understanding the submicroscopic world. Chemical education: Towards research-based practice. J. Gilbert, O. de Jong, R. Justi, D. F. Treagust and J. van Driel. Dordrecht, Kluwer Academic Publishers: 189-212.

23. Harrison, A. G., Treagust, D. F. (2000). "Learning about atoms, molecules, and chemical bonds: A case study of multiple-model use in grade 11 chemistry". Science Education 84(3): 352-381.

24. Hibbard, K. M., Novak, J. D. (1975). "Audiotutorial elementary school science instruction as a method for study of children's concept learning: Particulate nature of matter". Science Education 59(4): 559-570.

25. Hrepic, Z., Zollman, D., Rebello, S. (2002). Identifying students' models of sound propagation. Paper presented at 2002 Physics Education Research Conference. Boise, Idaho.

26. Hwang, B.-T., Liou, A.-W. (1993). "Study of the students' representation of the gas volume through the particulate conception". Paper presented at 34th IUPAC Congress, Beijing.

27. Jiménez Gómez, E., A. Benarroch, et al. (2006). "Evaluation of the degree of coherence found in students' conceptions concerning the particulate nature of matter". Journal of Research in Science Teaching 43(6): 577-598.

28. Johnston, K. (1990). Students' responses to an active learning approach to teaching the particulate theory of matter. Relating macroscopic phenomena to microscopic particles: a central problem in secondary Science Education. P. L. Lijnse, Licht, P., Vos, W. de, Waarlo, A. J. Utrecht, CD-B Press: 247-265. 
29. Keig, P. F., Rubba, P. A. (1993). "Translation of representations of the structure of matter and its relationship to reasoning, gender, spatial reasoning, and specific prior knowledge". Journal of Research in Science Teaching 30(8): 883-903.

30. Kelly, G., Chen, C. (1998). "The sound of music: Experiment, discourse, and writing of science as sociocultural practices". Paper presented at the annual meeting of the American Educational Research Association, San Diego, April 1998: 158.

31. Kelly, G. J., Chen, C. (1999). "The sound of music: Constructing science as sociocultural practices through oral and written discourse". Journal of Research in Science Teaching 36(8): 883-915.

32. Klopfer, L. E., Champagne, A. B., Chaiklin, S. D. (1992). "The ubiquitous quantities: explorations that inform the design of instruction on the physical properties of matter". Science Education 76(6): 597-614.

33. Kokkotas, P., Vlachos, I., Koulaidis, V. (1998). "Teaching the topic of the particulate nature of matter in prospective teachers' training courses". International Journal of Science Education 20(3): 291-303.

34. Krnel, D. and S. S. Glazar (2003). "The development of the concept of "Matter": A crossage study of how children classify materials". Science Education 87(5): 621-639.

35. Kruger, C., Palacio, D., Summers, M. (1992). "Surveys of English primary teachers' conceptions of force, energy, and materials". Science Education 76(4): 339-351.

36. Kruger, C., Summers, M. (1989). "An investigation of some primary teachers' understanding of changes in materials". School Science Review 71(255): 17-27.

37. Lee, K.-W. L. (1999). "Particulate representations of a chemical reaction mechanism". Research in Science Education 29(3): 401-415.

38. Jing Lei, J., Zhao, Y. (2007). "Technology uses and student achievement: A longitudinal study". Computers \& Education 49: 284-296.
39. Leite, L., Afonso, A. (2001). Portuguese school textbooks' illustrations and students' alternative conceptions on sound. Physics Teacher Education Beyond 2000. R. Pinto, Surinach, S. Paris, Elsevier: 167-168.

40. Lesh, R., Doerr, H. (2003). Foundations of a models and modelling perspective on mathematics teaching, learning and problem solving. In R. Lesh \& H. Doerr (Eds.) Beyond constructivism: A models and modelling perspective on mathematics problem solving, learning and teaching. Mahwah, NJ: Lawrence Earlbaum.

41. Lichtfeldt, M. (1996). Development of pupils' ideas of the particulate nature of matter: Long-term research project. Research in Science Education in Europe. G. Welford, Osborne, J., Scott, P. London, The Falmer Press: 212-228.

42. Linder, C. J. (1987). Tertiary physics: a case study in students' conceptions of sound. Proceedings of the 2. Int. Seminar "Misconceptions and Educational Strategies in Science and Mathematics", Vol. III. J. Novak. Ithaca, Cornell University: 322-334.

43. Linder, C. J. (1992). "Understanding sound: so what is the problem?" Physics Education 27(5): 258-264.

44. Linder, C. J. (1993). "University physics students' conceptualizations of factors affecting the speed of sound propagation". International Journal of Science Education 15(6): 655-662.

45. Linder, C. J., Erickson, G. L. (1989). "A study of tertiary physics students' conceptualizations of sound". International Journal of Science Education 11: 491-501.

46. Ma-Naim, C., Finkental, M. (2001). The initiation of thermodynamic theory from the particulate model for preservice teachers. Physics Teacher Education Beyond 2000. R. Pinto, Suriñach, S. Paris, Elsevier: 301-304.

47. Magnusson, S. J. (1996). "Complexities of learning with computer-based tools: A case of inquiry about sound and music in elementary school". Journal of Science Education and Technologies 5(4): 297-309. 
48. Maurines, L. (1993). Spontaneous reasoning on the propagation of sound. Proceedings of the Third International Seminar on Misconceptions and Educational Strategies in Science and Mathematics. J. Novak. Ithaca, New York, Cornell University (distributed electronically).

49. Mazens, K. (1997). "Conceptual change in Physics: Naive representations of sounds in 6- to 10-year old children". Paper presented at the EARLI conference, Athens, August 1997: 1-9.

50. Mazens, K.; Lautrey, J. (2003). Conceptual change in physics: children's naive representations of sound. Cognitive Development, 18: 159-176.

51. Meheut, M., Chomat, A. (1990). The bounds of children's atomism: An attempt to make children build up a particulate model of matter. Relating macroscopic phenomena to microscopic particles: a central problem in secondary Science Education. P. L. Lijnse, Licht, P., Vos, W. de, Waarlo, A. J. Utrecht, CD-B Press: 266-282.

52. Novick, S., Nussbaum, J. (1978). "Junior High School pupils' understanding of the particulate nature of matter: An interview study". Science Education 62: 273-281.

53. Novick, S., Nussbaum, J. (1981). "Pupils' understanding of the particulate nature of matter: A cross-age study". Science Education 65: 187196.

54. Ogborn, J. (2002). Ownership and transformation: Teachers using curriculum innovations. Physics Education, 37 (2), pp.142-146.

55. Papalexopoulos, P. F., Patapis, S. (2001). Teaching new (smart) materials at schools. Children's ideas about materials. Physics Teacher Education Beyond 2000. R. Pinto, Suriñach, S. Paris, Elsevier: 237-239.

56. Pintó, R. (2005). Introducing curriculum innovations in science: Identifying teachers' transformations and the design of related teacher education. Science Education, 89(1), 1-12.

57. Pritchard, A. J. (1990). Biology education and particulate theory: "Too much chemicals!" Relating macroscopic phenomena to microscopic particles: a central problem in secondary Science Education. P. L. Lijnse, Licht, P., Vos, W. de, Waarlo, A. J. Utrecht, CD-ß Press: 131-138.
58. Pujol, R. (1993). The chemistry textbooks used by ninth grade Venezuelan students as possible sources of misconceptions about the structure of matter. Proceedings of the Third International Seminar on Misconceptions and Educational Strategies in Science and Mathematics. J. Novak. Ithaca, New York, Cornell University (distributed electronically).

59. Rohr, M., Reimann, P. (1997). "Reasoning with multiple representations when acquiring the particulate model of matter". Occasional paper, University of Heidelberg, Germany: 1-29.

60. Rohr, M., Reimann, P. (1999). A cognitive model to describe the process of learning the particulate model of matter and its instructional consequences. Research in Science Education Past, Present, and Future Vol.1. M. Komorek, Behrendt, H., Dahncke, H., Duit, R., Graeber, W., Kross, A. Kiel, IPN Kiel: 195-197.

61. Rosser, R., Narter, D. (1998). "What sort of stuff is sound? Children and adults intuitions about a physical phenomenon". Poster presented at the annual meeting of AERA, San Diego, 1998: 1-12.

62. Sanger, M. J. (2000). "Using particulate drawings to determine and improve students' conceptions of pure substances and mixtures". Journal of Chemical Education 77(6): 762-766.

63. Sanger, M. J. (2005). "Evaluating students' conceptual understanding of balanced equations and stoichiometric ratios using a particulate drawing". Journal of Chemical Education 82(1): 131-134.

64. Sarantopoulos, P., Pantidos, P., Patapis, S. (2001). Using analogy to represent a transverse wave. Proceedings of the Third International Conference on Science Education Research in the Knowledge Based Society, Vol. 2. D. Psillos, Kariotoglou, P., Tselfes, V., Bisdikian, G., Fassoulopoulos, G., Hatzikraniotis, E., Kallery, M. Thessaloniki, Greece, Aristotle University of Thessaloniki: 841-843.

65. Saura, O.; De Pro, A. (1999). ¿Utilizan los alumnos esquemas conceptuales en la interpretación del sonido? Enseñanza de las Ciencias, 17(2): 193-210. 
66. Scott, P. H. (1992). Pathways in learning science: A case study of the development of one student's ideas relating to the structure of matter. Research in physics learning: Theoretical issues and empirical studies. R. Duit, Goldberg, F., Niedderer, H. Kiel, IPN: 203-224.

67. Schibeci, R. A., Hickey, R. (2000). "Is it natural or processed? Elementary school teachers and conceptions about materials". Journal of Research in Science Teaching 37(10): 1154-1170.

68. Segarra, P. (1993). Helping students to develop the concepts related with wave movement through laboratory experiences. Proceedings of the Third International Seminar on Misconceptions and Educational Strategies in Science and Mathematics. J. Novak. Ithaca, New York, Cornell University (distributed electronically).

69. Selley, N. J. (2000). "Students' spontaneous use of a particulate model for dissolution". Research in Science Education 30(4): 389-402.

70. Selley, N. J. (2001). "Students' spontaneous use of a particulate model for dissolution". Research in Science Education 30(4): 389-402.

71. Singer, J. E., R. T. Tal, et al. (2003). "Students' understanding of the particulate nature of matter". School Science and Mathematics 103(1): 28-44.

72. Snir, J., C. L. Smith, et al. (2003). "Linking phenomena with competing underlying models: A software tool for introducing students to the particulate model of matter". Science Education 87(6): 794-830.

73. Staver, J. (1998). "Constructism: Sound theory for explicating the practice of science and science teaching". Journal of Research in Science Teaching 35(5): 501-520.

74. Stavy, R. (1996). Children's conceptions of the states of matter. Teaching the science of condensed matter and new materials. M. Michelini, Jona, S., Cobai, D. Udine, Forum: 8194.

75. Treagust, D. F., Jacobowitz, R., Gallagher, J. L., Parker, J. (2001). "Using assessment as a guide in teaching for understanding: A case study of a middle school science class learning about sound". Science Education 85(2): 137-157.

76. Utges, G., Pacca, J. L. A. (2001). Analogical reasoning and meaningful learning. A discussion about the use od analogies in teaching the concept of wave. Physics Teacher Education Beyond 2000. R. Pinto, Suriñach, S. Paris, Elsevier: 289-292.

77. Velázquez-Marcano, A., V. Williamson, et al. (2004). "The use of video demonstrations and particulate animation in general chemistry". Journal of Science Education and Technology 13(3): 315-324.

78. Vince, J., Tiberghien, A. (2001). Modelling in teaching and learning elementary physics on sound. Proceedings of the Third International Conference on Science Education Research in the Knowledge Based Society, Vol. 1. D. Psillos, Kariotoglou, P., Tselfes, V., Bisdikian, G., Fassoulopoulos, G., Hatzikraniotis, E., Kallery, M. Thessaloniki, Greece, Aristotle University of Thessaloniki: 38-50.

79. Voorde, H. H. t. (1990). On teaching and learning about atoms and molecules from a van Hiele point of view. Relating macroscopic phenomena to microscopic particles: a central problem in secondary Science Education. P. L. Lijnse, Licht, P., Vos, W. de, Waarlo, A. J. Utrecht, CD- $B$ Press: 81-104.

80. Watt, D., Russell, T. (1990). Sound. Liverpool, Liverpool University Press.

81. Wightman, T., Green, P., Scott, P. H. (1986). Children's learning in science project. The construction of meaning and conceptual change in classroom settings: Case studies on the particulate nature of matter. Leeds, University of Leeds, Centre for Studies in Science and Mathematics Education.

82. Williamson, V., D. Huffman, et al. (2004). "Testing students' use of the particulate theory". Journal of Chemical Education 81(6): 891-896.

83. Williamson, V. M., Abraham, M. R. (1995). "The effects of computer animation on the particulate mental models of college chemistry students". Journal of Research in Science Teaching 32(5): 521-534. 
84. Wittmann, M., Steinberg, R. N., Redish, E. F (1999). "Making sense of how students make sense of mechanical waves". The Physics Teacher 37(1): 15-21.

85. Wittmann, M. C. (1998). Making sense of how students come to an understanding of physics: An example from mechanical waves. College Park, $\mathrm{MD}$, University of Maryland.
86. Wittmann, M. C. (2002). "The object coordination class applied to wave pulses: Analysing student reasoning in wave physics". International Journal of Science Education 24(1): 97-118.

87. Wittmann, M. C., Steinberg, R. N., Redish, E. F. (2003). "Understanding and affecting student reasoning about sound waves". International Journal of Science Education 25(8): 991-1013. 



\section{B: DESCRIPTION AND ANALYSIS OF STUDENT ACTIVITIES}




\section{B: DESCRIPTION AND ANALYSIS OF STUDENT ACTIVITIES}

The sequence "Acoustic Properties of Materials" has been structured in three main units, each with its own driving questions that constitute a Chapter:

Unit 1. Sound wave - material interaction (5h)

- Acoustic problems of a disco: What happens to sound inside and outside a disco? (Chapter 1.1).

- Why can sound reach any corner of the dance floor? (Chapter 1.2)

- How can we manage to avoid hearing too much sound outside the disco? (Chapter 1.3).

Unit 2. Properties and internal structure of sound reflectors and sound absorbers (5h)

- Which characteristics does a good sound reflector have? And a good sound absorber? (Chapter 2.1).

- How can we explain that the properties of a material affect its acoustic behaviour? (Chapter 2.2).

Unit 3. Acoustic conditioning and soundproofing (2h)

- Comparing materials. Which one could be used to soundproof? (Chapter 3.1).

\section{Recommended Settings and Pedagogical Approach}

The whole sequence is student-centred and is intended to engage students in group activities, except those activities which require an individual reflection on one's own learning or those that ask for a discussion within the whole class. The role of the teacher is to guide students, stating explicitly the aims of each task or reformulating and adapting new key questions in order to help them to find their own learning path. This teacher's role as a facilitator is necessary to promote a gradual development of students' autonomy when questioning, thinking, planning, reflecting, interacting, discussing, and gradually developing conceptual frameworks through the active participation in tasks, with or without an experimental component.

The Sequence of concepts and the Learning targets for each block (Unit 1, 2 and 3) are described in detail below. The sequence of teaching and learning activities designed to promote the intended learning targets from each block is also shown. Some recommendations (Discussion of the experiments and exercises) about each of the activities included in each of the Chapters are provided and also some comments about the Equipment required. These descriptions are numbered using the same number of the materials for students (Student Activity Sequence).

\section{UNIT 0: INTRODUCTION TO ACOUSTICS}

The sequence is introduced by means of Chapter 0 , which poses the problem of noise pollution and emphasizes the role of science and technology in solving this kind of problem. This Chapter is also aimed at making students aware of the importance of using an accurate scientific language to allow us to communicate efficiently. For this reason, this Chapter revises the terminology associated to sound and summarizes the basic concepts related to sound nature and sound propagation that are required as a pre-requisite of the whole sequence.

\section{Discussion of the Experiments and Exercises}

At the beginning of this Chapter, it would be advisable that teachers encouraged students to begin discussing why the topic "Acoustic properties of materials" generates interest of study in order to stimulate their own interest and feeling of usefulness of the contents which are dealt in the sequence.

0.1. Students should work in pairs and later, a whole class discussion would be beneficial. The main aim of this activity is to make students aware of the difficulty of describing sounds in a way that allows other people to exactly reproduce the described sounds. In consequence, the activity is aimed at making students aware of the necessity of describing sounds with a more accurate language, in terms of certain magnitudes.

0.2. In this exercise, students have to explore the relationships between scientific and everyday language related to acoustics.

After these two exercises, there is a text which summarizes and stresses the key concepts which are necessary as pre-requisites of the sequence. It would be necessary that students revised these basic concepts related to sound since the notions related to the nature of sound and sound propagation are dealt throughout the whole sequence. Nevertheless, if students have not been taught previously about sound and this sequence represents their first approach to sound, some extra activities or resources should accompany this text to analyze each of the basic concepts that are discussed in this first Chapter. For instance, there are some interactive simulations (such as SimulaSON or the one developed within the PhET Project, see chapter 10 in section A) that could be very 
useful for this purpose. Other physical resources which are often used and experiments which turn out to be significant for students to learn about sound are:

- A slinky to teach/learn about the propagation of waves,

- An experiment which consists of placing a sound source inside a recipient to remove from there air molecules leaving behind a partial vacuum so that students can analyze the relationship between sound and the presence of a medium.

- It is also helpful to bring tuning forks with different frequencies into class to analyze the relation between frequency (and wavelength) and "speed of vibration".

- Teachers could also bring sound level meters to class so that students get used to using instruments to measure certain magnitudes related to sound (sound intensity level in this case).

\section{Equipment}

No special equipment is required. It will depend on the needs of each class group.

\section{UNIT 1: SOUND WAVE - MATERIAL INTERACTION}

\section{Sequence of contents}

Phenomena related to sound wave - material interaction that affects to sound propagation: reflection, diffuse reflection, reverberation.

Phenomena related to sound wave - material interaction that affects to sound attenuation: reflection, absorption, attenuation.

Model of sound attenuation in terms of the distribution of energy associated to sound: energy of incident sound, energy of reflected sound, energy of transmitted sound, absorbed energy.

Principle of conservation of energy applied in sound attenuation phenomena.

Sound attenuating materials depending on their acoustic behaviour: sound reflectors, sound absorbers, sound insulators.

\section{Learning Targets}

At the end of Unit 1, students should be able to...

1. Recognize the importance of using an appropriate scientific vocabulary to describe sounds.

2. Use scientific terminology to talk about sounds and sound phenomena.

3. Relate one's own experiences related to sound and noise pollution with scientific knowledge.

4. Read and interpret images related to sound reflection.

5. Explain certain situations in terms of the sound reflection phenomenon.

6. Draw the direction of the reflected sound wave knowing the angle of the incident sound wave and using the law of the angles.

7. Explain certain situations in terms of reverberation of sound

8. Interpret sound reflection on irregular surfaces in terms of sound diffusion.

9. Distinguish clearly between reverberation and echo phenomena.

10. Interpret sound attenuation in materials in terms of energy distribution. 
11. Apply the principle of conservation of energy when expressing that the energy of an incident sound in a closed space is distributed between the energy of the reflected sound, the energy of the transmitted sound and the absorbed energy within a material.

12. Express and apply in different contexts the diagram that describes that an incident sound on an interface is partly reflected, partly absorbed and partly transmitted through a material.

13. Relate the sound intensity level measured with the quantity of energy that is associated to the sound wave.

14. Associate the sound intensity level decrease through a wall or any other obstacle to sound attenuation.

15. Distinguish the meaning of sound absorber from the meaning of sound reflector according to their acoustic behaviour.

16. Design and carry out an experiment to measure the attenuation of sound caused by a material.

17. Relate the sound intensity level measured in a certain point of the space with the distance from that point to the sound source.

18. Control the variables of an experiment.

19. Measure the sound intensity level using a sound level meter.

20. Relate the intensity level of perceived sound with the respective values observed in a graph of sound intensity level vs. time.

21. Predict the evolution of a phenomenon and compare the prediction with the obtained result and with the conceptual model.

22. Interpret the meaning of graphs of sound intensity level vs. time.

23. Calculate sound attenuation as the subtraction between the incident sound and the transmitted sound through a material.

\subsection{ACOUSTIC PROBLEMS OF A DISCO}

This Chapter is an introduction to the context of the sequence, which deals with the problems of a disco which is not very well soundproofed.

\section{Discussion of the Experiments and Exercises}

It is important to let students read and discuss the problems related to the context of the disco since it is the scenario in which problems are posed and questions are raised throughout the whole sequence.
1.1.1. This activity proposes the observation of a disco (by means of photographs) to encourage students to discuss and identify the acoustic necessities of each area of a certain disco.

1.1.2. This activity can be used as a "pre-test" since it is intended to explore students' previous ideas about phenomena related to sound (reflection, diffraction ${ }^{2}$, and attenuation) through questions contextualized in the disco scenario.

\section{Equipment}

No special equipment is required. Nevertheless, the images of the disco could be projected on a big screen to facilitate the observation of the different areas of the disco since the pictures shown in the students' worksheets are not very clear.

\subsection{WHY CAN SOUND REACH ANY CORNER OF THE DANCE FLOOR?}

This Chapter is intended for the study of sound reflection and other associated phenomena (such as reverberation and diffuse reflection) and different technological applications (such as surround sound systems).

\section{Discussion of the Experiments and Exercises}

1.2.1. This activity is aimed at promoting the exploration of previous ideas about sound reflection. Students are asked about their ideas involving reflection in smooth or uneven surfaces, and about the direction of propagation of reflected sound waves.

1.2.2. In this activity, students have to compare sound reflection with other phenomena involving reflection (such as light or mechanical rebounds after collisions). The purpose is to analyze the commonalities between these situations regarding the direction of propagation of the reflected waves or objects.

1.2.3. Students are provided with a set of experimental data about sound reflection in different directions and they have to reach a conclusion about the law of angles when sound is reflected on a surface.

1.2.4. Students are asked to summarize their conclusions on sound reflection: what reflection is and what angle the reflected sound waves form with the surface in relation to the incident sound waves.

2. Diffraction has not been included since the module is mainly focused in the phenomena that happen when sound modifies any of their properties due its interaction with a material. 
1.2.5. Students are asked to apply their knowledge on sound reflection and directions of propagation of reflected sound in a practical situation: the use of sound diffusers in different rooms allocated to different uses.

1.2.6. This activity is intended to explore students' previous ideas about sound reverberation.

1.2.7. Two graphs that show the time of reverberation in a room before and after being acoustically conditioned are provided. Students have to interpret the meaning of these graphs in terms of the effects that a listener would perceive (more or less duration of sounds) due to the reverberation in the room.

1.2.8. Students are provided with some data about time of reverberation in a theatre and they have to interpret this data in order to reach a sound conclusion.

One of the most important tasks of the teachers is to promote that students confront the explanations that they elaborate with the scientific point of view. Therefore, it is advisable that teachers engage their students in reflecting and discussing what science tell us about the reflection phenomenon (in this case) regarding their previous ideas.

On the other hand, students should learn that one's predictions do not need to be corrected but discussed and justified. Teachers should reinforce this view as a way of promoting and increasing students' reflection on their own explanations by discussing among their peers and rising debates.

\section{Equipment}

No special equipment is required. Again the images in exercise 1.2 .5 could be projected in a big screen to facilitate the observation of the rooms of the disco since the pictures shown in the students' worksheets are not very clear.

Some teachers have found a ripple tank to be useful to generate waves and allow students to experience the reflection of waves in water when they reach an obstacle. The simulation developed within the PhET Project can be also used with the same purpose.

\subsection{HOW CAN WE MANAGE TO AVOID HEARING TOO MUCH SOUND OUTSIDE THE DISCO?}

This Chapter is devoted to the study of sound attenuation through a material object in terms of the energy associated to sound. Once the phenomena related to sound attenuation has been analyzed, the difference between sound insulators, absorbers and reflectors is stated in terms of their acoustic behaviour. Discussion of the Experiments and Exercises

1.3.1. Students are asked some questions to allow teachers to explore students' previous ideas about sound attenuation.

1.3.2. This activity is the first time in the sequence that students have to use a data capture system and a sound level meter to measure the variations of sound intensity level. It would be necessary that students tried to understand and become familiar with the instruments, connections and setup as they will use the instrument in other experimental activities along the sequence. In order to interpret and predict appropriately graphs (sound intensity level vs. time) it is important to help students to identify the relevant aspects of a graph like the ones that are expected in this experiment (differences of sound intensity level of various sounds or values) and avoid the irrelevant ones (shape of the graph).

1.3.3. This experiment consists of the analysis of the empirical relation between sound intensity level and the distance from the sound source to the sound level meter. The graph that shows the relationship between sound intensity level and distance from the sound source can be obtained from the source up to a certain distance ( $2 \mathrm{~m}$ approximately). Further than that distance, sound intensity level remains almost constant inside a closed room. The speed of separation of the sound level meter from the source can highly affect the form of the obtained graph. It is advisable to move the sound level meter away from the sound source at a constant speed.

1.3.4. Students have to apply what they understand by attenuation in order to calculate sound attenuation from provided data. If students have an appropriate conception of what attenuation is, they will calculate sound attenuation as the subtraction between the incident sound and the transmitted sound through a material.

1.3.5. Students have to explain the phenomena they associate to sound attenuation.

1.3.6. From a visual representation of how two different materials behave in front of sound in terms of how they distribute the energy associated to sound 
when they interact, students have to interpret which material behaves as a sound reflector and which behaves as a sound absorber.

1.3.7. Students are asked to design an experiment to determine if a material attenuates sound a lot or a little. It is highly advisable that students share and discuss their experimental designs with their teacher or within the whole class because often, in little groups, students quickly come to a consensus about what experiment they are going to perform but have not justified it deeply, so they need some key questions that make them reflect on it further. There are some experimental issues that should be discussed before performing the experiments. For instance:

- Teachers should emphasize the importance of keeping some variables constant such as the distance between the sound source and the sound level meter. - The sound source which is chosen for the experiment should also be properly selected since it is necessary to perform the experiment with a sound source which provides a constant sound intensity level.

1.3.8. Students have to carry out the designed experiment. In previous classroom implementations of the sequence, some students decided to measure sound attenuation caused by closing a door which separated the classroom and the corridor, and comparing this level with the level obtained when the door was open. Other students decided to use a box or a closed recipient as a smaller model of a room and measure sound intensity level when the box is open and closed.

1.3.9. The last exercise of Unit 1 consists of a selfassessment followed by a peer-assessment. Students are asked a general question so that they can reflect and try to relate all the concepts that have been studied throughout this first Unit of the sequence. Furthermore, students have to contribute to help their classmates find answers. It would be very beneficial for students that teachers also revised the answers that students elaborate in order to provide them some feedback and try to enrich their conceptual connections.

\section{Equipment}

According to the designers' proposal, activities 1.3.2 and 1.3.3 require a sound level meter connected to a computer which has previously installed software to collect and analyze data. In the design context, it was used MBL technology (sensor + interface). In these activities, it was decided to use the sensor (sound level meter) connected to a computer because this equipment facilitates the visualization of a graph which illustrates all the variations of sound intensity level that correspond to the perceived sounds. On the contrary, the sound level meter by itself only shows the changing values of sound intensity level at each moment but it cannot save data.

In activity 1.3.8, the sound level meter is also required to measure sound intensity level but this time the sensor could be used without being connected to the computer because students need to select a single value. Moreover, depending on the experiment they have designed, the fact of having the instrument not connected by cables to the computer let them move around the class or other spaces to perform their experiment. In this activity, students would also need a sound source which is constant in its intensity level. Therefore, students could use a mobile phone which plays a constant sound or a buzzer. 
UNIT 2: PROPERTIES AND INTERNAL STRUCTURE OF SOUND REFLECTORS AND SOUND ABSORBERS

\section{Sequence of Contents}

Model of sound absorber in terms of its physical properties (hardly rigid, hardly dense, porous).

Model of sound reflector in terms of its physical properties (rigid, dense, non porous).

Model of sound absorber and sound reflector in terms of their internal structure.

Model to explain the mechanisms of sound attenuation (by reflection and by absorption) of sound absorbers and reflectors in terms of their physical properties (rigidity, density and porosity) and their internal structure.

\section{Learning Targets}

At the end of Unit 2, students should be able to...

24. Prepare an experimental setting to measure and collect data of sound intensity level.

25. Compare numerical values obtained empirically in order to come to some conclusions.

26. Distinguish the meaning of sound absorber from the meaning of sound reflector depending on the sound intensity level that is measured inside a box covered with these materials.

27. Compare their preconceptions about acoustic properties of materials with the elaborated conceptual models and distinguish the differences.

28. Design an experiment to determine whether a material is a sound reflector or a sound absorber, controlling the dependent variables.

29. Use the properties of a material to predict whether a material will behave as a sound absorber or a sound reflector.

30. Relate the fact that a material is a sound reflector or sound absorber with the combination of certain properties: numerical value of its density, degree of stiffness noticed by direct manipulation, its porosity observed with a binocular microscope.

31. Use a conceptual model of the microstructure to represent a dense material.

32. Use a conceptual model that allows relating the density of a material with its acoustic behaviour.

33. Use a conceptual model of the microstructure to represent a rigid material.
34. Use a conceptual model that allows relating the rigidity of a material with its acoustic behaviour.

35. Represent a porous material in terms of its internal structure.

36. Use a conceptual model that allows relating the porosity of a material with its acoustic behaviour.

37. Use a conceptual model to interpret mechanisms of sound attenuation in materials in terms of energy transfer or energy dissipation.

\subsection{WHICH CHARACTERISTICS DOES A GOOD SOUND REFLECTOR HAVE? AND A GOOD SOUND ABSORBER?}

This Chapter consists of a guided inquiry-oriented activity sequence that promotes the elaboration of a model of a sound reflector and sound absorber in terms of their physical properties.

\section{Discussion of the Experiments and Exercises}

2.1.1. This exercise is an open-ended question that is intended to explore students' previous ideas about the characteristics or physical properties that sound attenuating materials have.

2.1.2. This activity also is an exploratory question about the properties that students associate to sound attenuating materials. Nevertheless, in this activity students have to explain why they assign certain characteristics to sound reflectors and sound absorbers. The main aim of this activity is to formulate a preliminary model of a sound absorber and sound reflector in terms of their physical properties that allows them to predict and test it after some experiments.

2.1.3. Students are asked to predict the acoustic behaviour of certain materials according to the preliminary model of their properties. It would be advisable for students to observe and manipulate a piece of each material before predicting their acoustic behaviour.

2.1.4. Students have to design an experiment to test if a material behaves as a sound reflector or as a sound absorber. A key question for triggering students' thinking on the experimental design is: "If you covered the walls of the disco (or the walls of your classroom) with pieces of a sound reflecting material (or sound absorbing material), what you would hear inside the closed space?" (In relation to the ordinary walls). 
2.1.5. Students have to carry out the experiment they have designed. To do it, boxes covered inside with different materials are provided to them. For doing so, they have to take into account that they need a reference value to compare their measurements of the sound intensity level. The reference value corresponds to the measurement of the sound intensity level inside a cardboard box without being covered by any material. Based on the experimental results, students are asked to classify the tested materials in two categories: sound reflectors and absorbers. If the sound intensity level that students measure inside the box covered by a certain material increases in relation to the reference value, it means that the material which covers the box is a sound reflector. On the contrary, if the sound intensity level decreases, it means that the material that has been used for covering the box is a sound absorber. At the end of this activity, it would be advisable for the whole class to share their results in order to compare their experimental results and come to appropriate conclusions about the acoustic behaviour of each material.

2.1.6. Once students have classified the tested materials distinguishing sound reflectors from sound absorbers, they have to identify which properties are common to all the sound reflectors used in the previous experiment and which properties are common to all the sound absorbers. Students are asked to generalize the properties of sound absorbers and sound reflectors. The main aim is to make students aware that some properties of materials are related to their acoustic behaviour and other properties are not.

After the classification of properties, students are asked to distinguish properties which are specific of materials from those which are for general objects. This sequence is aimed at acoustic properties of materials but it does not deal with the influence of other properties of objects, such as thickness or surface material pieces. In order to avoid communicating the idea that these factors do not affect the acoustic behaviour of materials (because they do so), teachers should emphasize that properties of objects (especially its geometry) can also affect the acoustic behaviour of a piece of material and this influence could be also analyzed even it is not addressed in the sequence.

Finally, based on these new evidences, students are asked to revise the preliminary model elaborated in activity 2.1.2 which relates the acoustic behaviour of materials to their physical properties.

2.1.7. Students are provided with some empirical data (such as the measurement of density of the tested materials) and they also have to observe and manipulate the previous sound reflectors and sound absorbers to obtain accurate data about their physical properties.

2.1.8. Finally, students should come to a conclusion about the physical properties of sound absorbers and sound reflectors.

2.1.9. This exercise is aimed at applying the previously refined conceptual model which relates acoustic behaviour of materials to their physical properties. Students are asked to predict how different materials would behave in front of sound according to the physical properties they can identify from the pictures of these materials.

\section{Equipment}

In activity 2.1.3, it would be advisable to provide students with some samples or pieces of materials that students can observe and manipulate in order to predict their acoustic behaviour.

In activity 2.1.5, six cardboard boxes are used. Five of them are covered inside with different materials (aluminium foil, felt, polyurethane, chipboard with Formica and glass wool). The remaining box does not need to be covered by any material because it is used to measure the reference value to which the rest of the measurements should be compared.

In activity 2.1.7, students are provided with technical sheets of each material (see Annex 1) to allow them to observe the internal structure of each tested material. The students are also asked to describe the materials so they should once again have some samples of the materials. 


\subsection{HOW CAN WE EXPLAIN THAT THE PROPERTIES OF A MATERIAL AFFECT ITS ACOUSTIC BEHAVIOUR?}

This Chapter is aimed at promoting the elaboration of a model of the internal structure of sound reflectors and absorbers that allows interpreting their acoustic behaviour according to their properties.

\section{Discussion of the Experiments and Exercises}

2.2.1. Students are asked some questions to guide them to use the particulate model of matter when conceptualizing density as a property of materials at a microscopic level. An analogy is introduced in the sequence: particles that form the materials or any other medium are compared with pool table balls. It is necessary that teachers explain the potential and limitations of this analogy.

2.2.2. In this exercise, students are supposed to transfer the language and reasoning used with the previous analogy and focus on particles to explain how density of materials can be conceptualized at the level of their internal structure and how density of materials affects their acoustic behaviour.

2.2.3. Students have to use the same analogy (pool balls) to interpret how to conceptualize rigidity as a property of materials at a microscopic level.

2.2.4. In this exercise, the students are supposed to transfer the language and reasoning used with the analogy and focus on particles to explain how rigidity of materials can be conceptualized at the level of their internal structure and how rigidity of materials affects their acoustic behaviour.

After these four activities, it would be necessary to discuss the limitations of the analogy used (e.g., it is important to clarify that density and rigidity are not independent properties but they are related. More density means higher atomic weight in some cases and/or a different arrangement of particles, but it also means different bonds among particles. The model used for explaining density is only useful for homogeneous materials but does not account for the differences between amorphous materials. Regarding the model for rigidity, the students should understand that the bonds among particles are not physical as it is represented with springs).
2.2.5. Students have to interpret how porosity of a material affects its acoustic behaviour.

2.2.6. Students are asked to summarize the physical properties that sound reflectors usually have and to apply the model that relates physical properties to the internal structure of materials to account for how sound is attenuated when it reaches a sound reflector.

2.2.7. Students are asked to interpret some experimental results based on the model which relates acoustic behaviour of materials to their internal structure.

\section{Equipment}

No special equipment is required.

\section{UNIT 3: ACOUSTIC CONDITIONING AND SOUNDPROOFING}

\section{Sequence of Contents}

Applications of sound reflectors, absorbers and insulators:

- Acoustic conditioning

- Soundproofing

\section{Learning Targets}

At the end of Unit 3, students should be able to...

38. Apply the conceptual model which relates the physical properties of materials to their acoustic behaviour in order to predict how new materials would behave with regard to sound attenuation.

39. Describe materials.

40. Autonomously design an experiment, using a sound level meter, to empirically test the acoustic behaviour of a material.

41. Distinguish between a sound absorber and a sound reflector.

42. Determine how much sound intensity a certain material attenuates.

43. Explain, using acoustic and non-acoustic criteria, their decisions about which materials are appropriate to use in order to achieve the acoustic conditions that a certain space requires.

44. Propose solutions to solve a problem related to soundproofing.

45. Summarize information and establish conclusions relating acoustic behaviour of some materials to their physical properties. 


\subsection{COMPARING MATERIALS. WHICH ONE COULD BE USED TO SOUNDPROOF?}

This Chapter was conceived as an open inquiryoriented sequence of activities which is driven by the question: which material would be the best sound absorber to be used for soundproofing a disco? This sequence of activities is proposed as a group project in which the students have to put into practice their knowledge on acoustic properties of materials and their skills to propose particular solutions to soundproof the disco (original problem).

\section{Discussion of the Experiments and Exercises}

3.1.1. Students are asked to describe the properties of certain materials by observing and manipulating them (and their technical sheets) and looking for further information on the Internet.

3.1.2. Students are asked to take into account different criteria that allow them to decide which material behaves as the best sound absorber. First of all, students have to predict which materials would behave as the best sound absorbers using the conceptual model which relates properties of materials to their acoustic behaviour.

3.1.3. Students are proposed to design and carry out an experiment to determine which material is the best sound absorber. To do so, students have to be provided with the boxes covered inside with different materials. They are expected to take different types of measurements. First of all, they should distinguish which materials from those provided behave as sound absorbers and which ones behave as sound reflectors. Therefore, they should perform the same experiment as the one carried out in activity.

3.1.5. On the other hand, students should determine which material behaves as the best sound absorber and so, they should measure which material attenuates sound more. Therefore, students should carry out a second experiment which consists of measuring the sound intensity level outside each box. In this case, the reference intensity value to be compared with the rest of the measurements would correspond to the sound intensity level measured outside the cardboard box without being covered by any plate of material.

3.1.4. Based on the previous results and on other relevant criteria, students have to reach an agreement about which sound absorber would be more useful to attenuate sound.

3.1.5. This activity consists of writing a report which collects all the information that allow students to decide which material is the best sound absorber to be used when soundproofing the disco. It would be worthwhile for students to present their group reports of the project in front of their classmates, stating their points of view, justifying their proposals and trying to reach a class agreement.

This activity sequence provides teachers with a good opportunity to monitor their students' progress and application of the (conceptual and procedural) acquired knowledge. It is necessary that students can have enough autonomy to work and discuss in groups, when predicting, designing and performing the experiment and bringing conclusions.

\section{Equipment}

In activity 3.1.1, students are provided with technical sheets of each material (see Annex 1) to allow them to observe the internal structure of each tested material. The students are also asked to describe the materials, so they should have some samples of the materials to manipulate and observe them.

In activity 3.1.3, nine cardboard boxes are used. Eight of them are covered inside with different materials and the remaining box does not need to be covered by any material because it is used to measure the reference values to which the rest of the measurements are to be compared. 


\section{C: EVALUATION TASKS}




\section{C: EVALUATION TASKS}

A broad range of strategies and instruments has been proposed to assess student learning outcomes. The assessment tasks include:

- Certain activities selected from the student activity sequence (formative assessment).

- A final test that assesses most of the learning targets which were intended for this sequence (summative assessment).

\section{FORMATIVE ASSESSMENT}

Some activities of the sequence have been designed to allow students to express their previous ideas on certain concepts or phenomena. Therefore, teachers can use them as a way of exploring a starting point for students. For instance:

1.1.2: Deals with sound-material interaction (sound reflection, sound transmission, sound diffraction, and sound attenuation).

1.2.1: Deals with sound reflection on different surfaces and direction of propagation of reflected sound.

1.2.6: Deals with the phenomenon of reverberation.

1.3.1: Deals with sound transmission and sound attenuation.

1.3.5: Deals with phenomena related to sound attenuation (absorption and reflection).

2.1.1: Deals with physical properties of sound reflectors and sound absorbers.

2.1.2: Deals with the relationship between acoustic behaviour of materials and their physical properties and internal structure.

Some activities of the sequence have been designed to encourage students to elaborate their conclusions after a set of activities. Therefore, teachers can use them as a way of exploring students' learning progress along the sequence. For instance:

1.2.4: Deals with reflection.

1.3.9: Deals with sound attenuation and acoustic behaviour of materials. This activity consists of a self-assessment activity followed by a peerassessment.

2.1.9: Deals with the conceptual model which relates physical properties of materials to acoustic behaviour.

2.2.7: Deals with the conceptual model which relates internal structure of materials to acoustic behaviour.

3.1.5: Was conceived as an application project. Students are asked to use the previous conceptual models to predict the acoustic behaviour of some materials. This project also involves assessing some procedural skills (such as, designing experiments with specific purposes or using experimental data to interpret evidence and reaching conclusions).

\section{SUMMATIVE ASSESSMENT}

The established partnership designed a final test, as an instrument that allows teachers (and researchers too) to assess most of students' learning outcomes that were stated when designing the sequence. After the first classroom implementation of the sequence, some rubrics for analyzing students' answers were also elaborated. These rubrics establish a gradation of students' answers depending on the appropriateness of each answer (from more to less appropriate answers). The questions of this final test and their corresponding rubrics are shown below: 


\section{QUESTION 1}

The images show two different perspectives of the same office. Two people work there and are separated by a screen. The problem is that in spite of the screen, they disturb each other when they talk on the phone. They want to know how sound emitted by the person on the left reaches the person on the right.
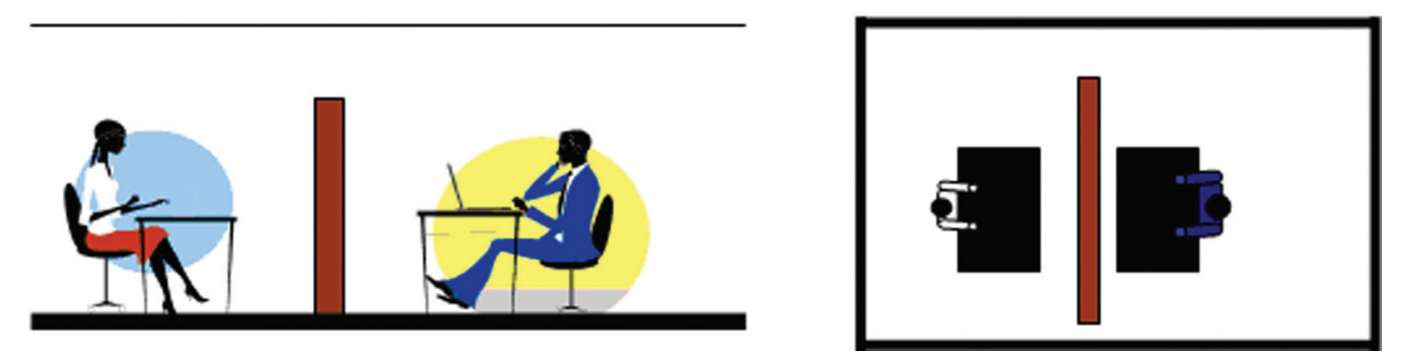

(a) Represent the path that sound waves travel from one person to the other, using both the wavefront and "sound ray" representations. Mention one of the phenomena which make it possible for these two people to hear each other. Describe this phenomenon.

(b) How would this office be if there was reverberation? What would the employees hear?

(c) An expert on acoustics tells them that a possible solution to avoid hearing too much sound is placing sound diffusers and sound absorbers. How do you account for the fact that these materials can be a solution?

\section{Rubrics (Q1)}

Q1 is intended to assess whether students have achieved the following learning targets, that is, if they are able to:

4. Read and interpret images related to sound reflection.

5. Explain certain situations in terms of the sound reflection phenomenon.

6. Draw the direction of the reflected sound wave knowing the angle of the incident sound wave and using the law of the angles.

7. Explain certain situations in terms of sound reverberation.

8. Interpret sound reflection on irregular surfaces in terms of sound diffusion.

9. Distinguish clearly between reverberation and echo phenomena.

10. Interpret sound attenuation in materials in terms of energy distribution.

15. Distinguish the meaning of sound absorber from the meaning of sound reflector according to their acoustic behaviour. 
FOR PART (A):

\begin{tabular}{|l|l|l|}
\hline & CATEGORY OF STUDENT'S RESPONSE & EXAMPLE OF STUDENT RESPONSE \\
\hline 1 & $\begin{array}{l}\text { The representation and/or the given explanation } \\
\text { illustrates that students take into account the law of } \\
\text { angles with regards to sound reflection. } \\
\begin{array}{l}\text { Furthermore, students are able to explain the } \\
\text { phenomenon of sound reflection. }\end{array}\end{array}$ & $\begin{array}{l}\text { Representation: When sound waves reach an } \\
\text { obstacle (ceiling or walls in this case), they are } \\
\text { partly reflected in a way that the direction of } \\
\text { propagation of reflected waves forms an angle with } \\
\text { the surface equal to the angle that incident waves } \\
\text { form with the ceiling (law of angles). } \\
\text { Reflection: It is a phenomenon in which waves } \\
\text { change their direction of propagation when } \\
\text { reaching an object but they continue propagating } \\
\text { through the same medium. }\end{array}$ \\
\hline 2 & $\begin{array}{l}\text { The representation and/or explanation provided } \\
\text { illustrates that students do not take into account } \\
\text { the law of angles with regards to sound reflection } \\
\text { but they consider that direction of propagation of } \\
\text { incident sound and reflected sound form a straight } \\
\text { angle. However, students are able to explain the } \\
\text { phenomenon of sound reflection. }\end{array}$ & $\begin{array}{l}\text { Representation: Drawing of incident and reflected } \\
\text { "sound rays" forming an angle of 90. } \\
\text { Reflection: It is a phenomenon in which waves } \\
\text { change their direction of propagation when } \\
\text { reaching an object but they continue propagating } \\
\text { through the same medium. }\end{array}$ \\
\hline 3 & \begin{tabular}{l} 
Irrelevant or inconsistent answers \\
\hline
\end{tabular} & \\
\hline
\end{tabular}

FOR PART (B):

\begin{tabular}{|l|l|l|}
\hline & CATEGORY OF STUDENT'S RESPONSE & EXAMPLE OF STUDENT RESPONSE \\
\hline 1 & $\begin{array}{l}\text { The explanation provided illustrates that students } \\
\text { consider that reverberation depends on the } \\
\text { characteristics of the room (height of ceilings, } \\
\text { objects and furniture). Furthermore, students } \\
\text { distinguish between reverberation and echo. }\end{array}$ & $\begin{array}{l}\text { The room would have a high and smooth ceiling } \\
\text { and the room would not have too many pieces of } \\
\text { furniture. } \\
\text { The employees would hear that sounds have more } \\
\text { duration so that their conversations would be less } \\
\text { intelligible (because reflected sound would have a } \\
\text { short delay in arriving to the listener with regards } \\
\text { to direct sound). }\end{array}$ \\
\hline 2 & $\begin{array}{l}\text { The explanation provided illustrates that students } \\
\text { consider that reverberation depends on the } \\
\text { characteristics of the room (height of ceilings, } \\
\text { objects and furniture). However, students do not } \\
\text { make a clear distinction between echo and } \\
\text { reverberation. }\end{array}$ & $\begin{array}{l}\text { The room would have a high and smooth ceiling } \\
\text { and the room would not have too many pieces of } \\
\text { furniture. } \\
\text { The employees would hear their own voices in } \\
\text { different moments since sound waves are reflected } \\
\text { several times and reach the listener at different } \\
\text { times. }\end{array}$ \\
\hline 3 & \begin{tabular}{l} 
Irrelevant or inconsistent answers \\
\hline
\end{tabular} & \\
\hline
\end{tabular}


FOR PART (C):

\begin{tabular}{|l|l|l|}
\hline 1 & CATEGORY OF STUDENT'S RESPONSE & EXAMPLE OF STUDENT RESPONSE \\
\hline 1 & $\begin{array}{l}\text { Students' answers demonstrate that they are able } \\
\text { to explain the acoustic behaviour of sound } \\
\text { diffusers and sound absorbers. Moreover, they } \\
\text { relate the behaviour of these materials or objects } \\
\text { with their function in sound attenuation in terms of } \\
\text { energy distribution. }\end{array}$ & $\begin{array}{l}\text { Sound diffusers have uneven surfaces so that they } \\
\text { reflect sound in multiple directions. This means } \\
\text { that they diffuse sound. Sound absorbers allow } \\
\text { sound propagation and dissipate part of the energy } \\
\text { of sound. Therefore, they decrease the amount of } \\
\text { sound reflection and increase the amount of sound } \\
\text { absorption. Both materials attenuate sound in } \\
\text { different ways: sound diffusers attenuate sound by } \\
\text { propagation (sound is distributed over the entire } \\
\text { space and less energy reaches each point) and } \\
\text { sound absorbers attenuate sound by absorption } \\
\text { within the material. }\end{array}$ \\
\hline 2 & $\begin{array}{l}\text { Students' answers demonstrate that they are able } \\
\text { to explain the acoustic behaviour of sound } \\
\text { diffusers and sound absorbers. However, they do } \\
\text { not explain their role in sound attenuation. }\end{array}$ & $\begin{array}{l}\text { Sound diffusers have uneven surfaces so that they } \\
\text { reflect sound in multiple directions. This means } \\
\text { that they diffuse sound. Sound absorbers allow } \\
\text { sound propagation and dissipate part of the energy } \\
\text { of sound. Therefore, they decrease the amount of } \\
\text { sound reflection and increase the amount of sound } \\
\text { absorption. }\end{array}$ \\
\hline 3 & Irrelevant or inconsistent answers & \multicolumn{2}{|c|}{} \\
\hline
\end{tabular}




\section{QUESTION 2}

(a) If you are outside a disco, you can hear part of the sound that comes from inside. The decrease of sound intensity level outside the disco in relation to the sound intensity level inside the disco is called:

(i) acoustic conditioning

(ii) attenuation

(iii) absorption

(b) This decrease of the sound intensity level could be due to:

(i) the absorption of part of the sound energy inside the materials

(ii) the reflection of sound in the surfaces it reaches on its way

(iii) both the absorption and reflection of sound

(c) Represent the energy of the reflected sound wave, the absorbed energy and the energy of the transmitted sound wave in a material $X$ which attenuates very little sound (bad sound absorber and bad sound reflector).

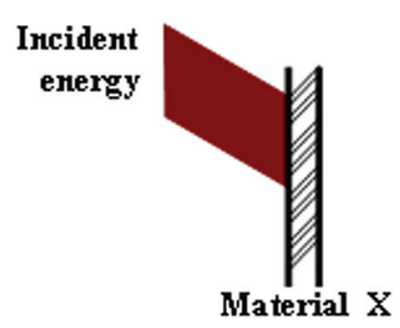

How would the attenuation of sound be caused by this material $X$ ?

If you were outside the disco and the walls were made material $X$, what would you hear? What level of sound intensity could be measured?

(d) Represent the energy of the reflected sound wave, the absorbed energy and the energy of the transmitted sound wave in a material $\mathrm{X}$ which is a very good sound absorber.

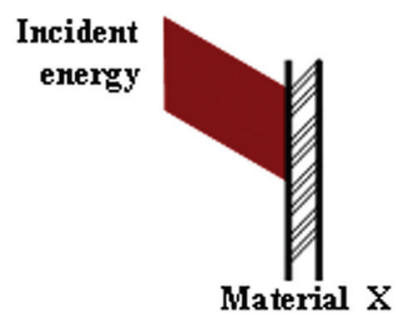

How would the attenuation of sound be caused by material $X$ ?

If you were outside the disco and the walls were made of material $X$, what would you hear? What level of sound intensity could be measured? 


\section{Rubrics (Q2)}

Q2 is intended to assess whether students have achieved the following learning targets, that is, if they are able to:

2. Use scientific terminology to talk about sounds and sound phenomena.

10. Interpret sound attenuation in materials in terms of energy distribution.

11. Apply the principle of conservation of energy when expressing that the energy of an incident sound in a closed space is distributed between the energy of the reflected sound, the energy of the transmitted sound and the absorbed energy within a material.

12. Express and apply in different contexts the diagram that describes that an incident sound on an interface is partly reflected, partly absorbed and partly transmitted through a material.

13. Relate the sound intensity level measured with the quantity of energy that is associated to the sound wave.

14. Associate the sound intensity level decrease through a wall or any other obstacle to sound attenuation.

15. Distinguish the meaning of sound absorber from the meaning of sound reflector according to their acoustic behaviour.

FOR PART (A) AND (B) OF Q2:

\begin{tabular}{|l|l|l|}
\hline & CATEGORY OF STUDENT'S RESPONSE & EXAMPLE OF STUDENT RESPONSE \\
\hline 1 & $\begin{array}{l}\text { Student's answer demonstrates that they are able } \\
\text { to use scientific terminology with an appropriate } \\
\text { meaning. }\end{array}$ & $\begin{array}{l}\text { Q2 (a) The correct choice is (ii) } \\
\text { Q2 (b) The correct choice is (iii) }\end{array}$ \\
\hline 2 & $\begin{array}{l}\text { Student's answer demonstrates that they are not } \\
\text { able to use scientific terminology with an } \\
\text { appropriate meaning. }\end{array}$ & Other choices \\
\hline
\end{tabular}

FOR PART (C) AND (D):

\begin{tabular}{|c|c|c|}
\hline & CATEGORY OF STUDENT'S RESPONSE & EXAMPLE OF STUDENT RESPONSE \\
\hline 1 & $\begin{array}{l}\text { Students represent the distribution of the energy } \\
\text { of an incident sound wave when interacting with a } \\
\text { material in terms of the reflected, absorbed and } \\
\text { transmitted energy, correctly identifying the } \\
\text { acoustic behaviour of the material. Moreover, the } \\
\text { representations demonstrate that students take } \\
\text { into account the principle of conservation of } \\
\text { energy. } \\
\text { Students' responses illustrate an appropriate } \\
\text { relationship between sound intensity level and the } \\
\text { quantity of energy that is associated to sound. } \\
\text { Furthermore, students' answers denote an } \\
\text { appropriate conceptualization of what sound } \\
\text { attenuation is. }\end{array}$ & $\begin{array}{l}\text { Representation: } \\
\text { (c) Little sound is reflected (represented as a thin } \\
\text { reflected sound "ray"). Very little energy is } \\
\text { absorbed (represented as a few "dots" inside the } \\
\text { material). Therefore, the transmitted sound "ray" is } \\
\text { thicker. } \\
\text { (d) Little sound is reflected (represented as a thin } \\
\text { reflected sound "ray"). More energy is absorbed } \\
\text { (represented as many "dots" inside the material). } \\
\text { Therefore, the transmitted sound "ray" is thinner. } \\
\text { These representations evidence that the principle } \\
\text { of energy conservation is taken into account (e. g., } \\
\text { energy of incident sound is distributed in three } \\
\text { components so that any of these components is } \\
\text { not represented with the same proportion as the } \\
\text { energy of incident sound). }\end{array}$ \\
\hline
\end{tabular}




\begin{tabular}{|c|c|c|}
\hline & & $\begin{array}{l}\text { Questions: } \\
\text { (c) The attenuation produced is low. Therefore, we } \\
\text { would hear a lot of sound (loud sound) outside a } \\
\text { disco which was covered with this material Thus, } \\
\text { the sound intensity level outside the disco would } \\
\text { be high. } \\
\text { (d) The attenuation produced is higher. Therefore, } \\
\text { we would hear less sound outside a disco which } \\
\text { was covered with this material. Thus, the sound } \\
\text { intensity level outside the disco would be low. }\end{array}$ \\
\hline 2 & $\begin{array}{l}\text { The representations demonstrate that students do } \\
\text { not take into account the principle of conservation } \\
\text { of energy. }\end{array}$ & $\begin{array}{l}\text { Representation: } \\
\text { These representations demonstrate that the } \\
\text { principle of energy conservation is not taken into } \\
\text { account (e. g., energy of incident sound is } \\
\text { distributed in three components but some of these } \\
\text { components are represented with the same } \\
\text { proportion (or more) as the energy of incident } \\
\text { sound). }\end{array}$ \\
\hline 3 & $\begin{array}{l}\text { Students represent the distribution of the energy } \\
\text { of an incident sound wave when interacting with a } \\
\text { material in terms of the reflected, absorbed and } \\
\text { transmitted energy, but identifying incorrectly the } \\
\text { acoustic behaviour of the material. }\end{array}$ & $\begin{array}{l}\text { Representation: } \\
\text { (d) For instance, students represent that the } \\
\text { material (which is considered a good sound } \\
\text { absorber) reflects a lot of sound and does not } \\
\text { absorb as much as it reflects. }\end{array}$ \\
\hline 4 & $\begin{array}{l}\text { Students do not represent an appropriate } \\
\text { distribution of the energy of an incident sound } \\
\text { wave when interacting with a material in terms of } \\
\text { the reflected, absorbed and transmitted energy, }\end{array}$ & $\begin{array}{l}\text { Representation: } \\
\text { Students do not draw some of the components in } \\
\text { which energy of sound waves is distributed. For } \\
\text { instance, they only represent reflected and } \\
\text { transmitted sound but not absorbed energy. }\end{array}$ \\
\hline 5 & $\begin{array}{l}\text { Students' responses evidence a lack of } \\
\text { understanding of what sound attenuation is. }\end{array}$ & $\begin{array}{l}\text { Questions: } \\
\text { Students do not distinguish between sound } \\
\text { attenuation and transmitted sound. Therefore, they } \\
\text { consider that the attenuation produced by a good } \\
\text { sound absorber is low. }\end{array}$ \\
\hline
\end{tabular}




\section{QUESTION 3}

A group of students have performed the same experiment that you made in class. They have used two boxes, each of them covered inside with a different material, to cover the sound source, and have measured the sound intensity level inside the box and outside the box. Look at their results and interpret them, answering the following questions.

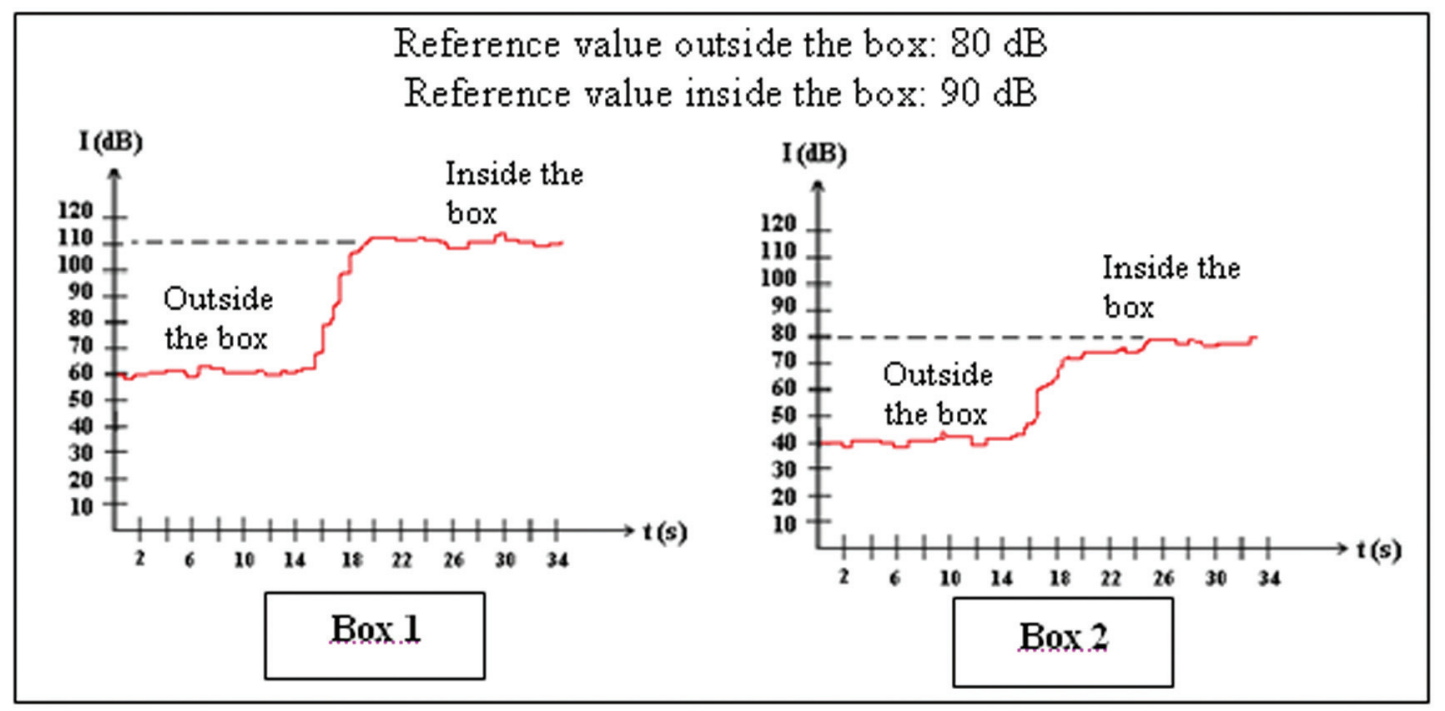

(a) Which box attenuates sound better? How much sound does each box attenuate?

(b) Which of the two boxes is covered inside with a sound absorber? Which box is covered inside with a sound reflector? Explain your answer.

(c) When these students measured the sound intensity level outside the box, they put the sound level meter at a certain distance from the box. What would happen if they measured the sound intensity level at a farther distance?

\section{Rubrics (Q3)}

Q3 is intended to assess whether students have achieved the following learning targets, that is, if they are able to:

13. Relate the sound intensity level measured with the quantity of energy that is associated to the sound wave.

14. Associate the sound intensity level decrease through a wall or any other obstacle to sound attenuation.

15. Distinguish the meaning of sound absorber from the meaning of sound reflector according to their acoustic behaviour.

17. Relate the sound intensity level measured in a certain point of the space with the distance from that point to the sound source.

22. Interpret the meaning of graphs of sound intensity level vs. time

23. Calculate sound attenuation as the subtraction between the incident sound and the transmitted sound through a material.

25. Compare numerical values obtained empirically in order to come to some conclusions.

26. Distinguish the meaning of sound absorber from the meaning of sound reflector depending on the sound intensity level that is measured inside a box covered with these materials. 
FOR PART (A):

\begin{tabular}{|l|l|l|}
\hline & CATEGORY OF STUDENT'S RESPONSE & EXAMPLE OF STUDENT RESPONSE \\
\hline 1 & $\begin{array}{l}\text { Student responses illustrate that sound attenuation } \\
\text { is appropriately conceptualized as the decrease of } \\
\text { sound intensity level when sound is transmitted } \\
\text { through a certain material. Therefore, students } \\
\text { appropriately identify the values of incident sound } \\
\text { in the graphs (reference value outside the box) and } \\
\text { transmitted sound (sound intensity level outside } \\
\text { the box covered with each material) to calculate } \\
\text { the attenuation produced by each material. }\end{array}$ & $\begin{array}{l}\text { Box } 1 \text { attenuates } 20 \mathrm{~dB}(80-60=20) \text {. Box } 2 \\
\text { attenuates } 40 \mathrm{~dB}(80-40=40) \text {. Therefore, the } \\
\text { attenuates sound more. }\end{array}$ \\
\hline 2 & $\begin{array}{l}\text { Students do not appropriately identify the values } \\
\text { of incident sound and transmitted sound to } \\
\text { calculate the attenuation produced by each } \\
\text { material. For instance, some students calculate the } \\
\text { difference between sound intensity level inside and } \\
\text { outside each box. Therefore, these students' } \\
\text { responses denote a lack of understanding of what } \\
\text { sound attenuation is. }\end{array}$ & $\begin{array}{l}\text { Box } 1 \text { attenuates } 50 \mathrm{~dB}(110-60=50) \text {. Box } 2 \\
\text { attenuates } 40 \mathrm{~dB}(80-40=40) \text {. Therefore, the } \\
\text { material that covers box } 1 \text { attenuates sound more. }\end{array}$ \\
\hline
\end{tabular}

FOR PART (B):

\begin{tabular}{|c|c|c|}
\hline & CATEGORY OF STUDENT'S RESPONSE & EXAMPLE OF STUDENT RESPONSE \\
\hline 1 & $\begin{array}{l}\text { Students' responses illustrate a clear distinction } \\
\text { between sound absorbers and sound reflectors } \\
\text { depending on the sound intensity level identified } \\
\text { in the graphs. Therefore, students compare the } \\
\text { values of sound intensity inside the two different } \\
\text { boxes with the reference value inside the box. } \\
\text { Moreover, students explain the acoustic behaviour } \\
\text { of each material relating sound intensity level with } \\
\text { the amount of energy associated to sound waves. }\end{array}$ & $\begin{array}{l}\text { The graph corresponding to box } 2 \text { shows that } \\
\text { inside this box the sound intensity level is lower } \\
\text { than the reference value (inside the box), which } \\
\text { indicates that the material that covers box } 2 \\
\text { absorbs part of the energy of the incident sound. } \\
\text { Therefore, we can say that box } 2 \text { is covered with a } \\
\text { sound absorber. } \\
\text { The graph corresponding to box } 1 \text { shows that } \\
\text { inside this box the sound intensity level is higher } \\
\text { than the reference value (inside the box), which } \\
\text { indicates that the material that covers box } 1 \\
\text { absorbs part of the energy of the incident sound. } \\
\text { Therefore, we can say that box } 1 \text { is covered with a } \\
\text { sound reflector. }\end{array}$ \\
\hline 2 & $\begin{array}{l}\text { Students identify (correctly or incorrectly) the } \\
\text { acoustic behaviour of materials in terms of the } \\
\text { sound attenuation that they produce. The idea that } \\
\text { sound absorbers allow more sound propagation } \\
\text { and so they attenuate less sound seems to be } \\
\text { related to the students' conception of sound as an } \\
\text { entity or substance that can go through materials. }\end{array}$ & $\begin{array}{l}\text { The material that covers box } 2 \text { attenuates sound } \\
\text { more. Therefore, this material is a sound reflector. }\end{array}$ \\
\hline
\end{tabular}


FOR PART (C):

\begin{tabular}{|c|l|l|}
\hline & CATEGORY OF STUDENT'S RESPONSE & EXAMPLE OF STUDENT RESPONSE \\
\hline 1 & $\begin{array}{l}\text { Students' responses illustrate that the sound } \\
\text { intensity level measured in a certain point of the } \\
\text { space is related to the distance from that point to } \\
\text { the sound source. }\end{array}$ & $\begin{array}{l}\text { The further the sound level meter is from the } \\
\text { sound source, the less sound intensity level is } \\
\text { measured. }\end{array}$ \\
\hline 2 & Irrelevant or inconsistent answers & $\begin{array}{l}\text { The further the sound level meter is from the } \\
\text { sound source, the more attenuated reflected } \\
\text { sound is because sound waves would become } \\
\text { attenuated through the air. }\end{array}$ \\
\hline
\end{tabular}




\section{QUESTION 4}

The same group of students continued inquiring about sound absorbers and sound reflectors.

(a) These students selected three different materials and determined their physical properties (density, rigidity and porosity). The following table includes the data and description of the properties of these materials:

\begin{tabular}{|c|c|c|c|}
\hline MATERIAL & DENSITY $(\mathbf{g} / \mathbf{c c})$ & RIGIDITY & POROSITY \\
\hline A & 0,02 & Rigid & No \\
\hline B & 0,07 & Flexible & Yes \\
\hline C & 3,00 & Rigid & No \\
\hline
\end{tabular}

According to their properties, which of these three materials do you think that would be the best sound reflector? Which of these materials would be the best sound absorber? Explain your answer.

(b) The students have also measured the sound intensity level putting a sound level meter inside and outside some boxes covered with these materials, and putting the sound source inside the box. The values obtained when measuring the sound intensity level are:

\begin{tabular}{|c|c|c|}
\hline MATERIAL & $\begin{array}{c}\text { SOUND INTENSITY LEVEL } \\
(\mathrm{dB}) \text { OUTSIDE THE BOX }\end{array}$ & $\begin{array}{c}\text { SOUND INTENSITY LEVEL } \\
(\mathrm{dB}) \text { INSIDE THE BOX }\end{array}$ \\
\hline D & $43-44$ & $68-69$ \\
\hline E & $69-70$ & $92-93$ \\
\hline F & $74-75$ & $82-83$ \\
\hline
\end{tabular}

Knowing that the reference value of sound intensity inside the box is $90 \mathrm{~dB}$, which of these values correspond to the best sound absorber? Which of the values correspond to the best sound reflector? Explain your answer.

(c) Which material attenuates more sound? Which material attenuates less sound? Explain your answer.

\section{Rubrics (Q4)}

Q4 is intended to assess whether students have achieved the following learning targets, that is, if they are able to:

13. Relate the sound intensity level measured with the quantity of energy that is associated to the sound wave.

14. Associate the sound intensity level decrease through a wall or any other obstacle to sound attenuation.

15. Distinguish the meaning of sound absorber from the meaning of sound reflector according to their acoustic behaviour.

25. Compare numerical values obtained empirically in order to formulate some conclusions.

26. Distinguish the meaning of sound absorber from the meaning of sound reflector depending on the sound intensity level that is measured inside a box covered with these materials.

30. Relate the fact that a material is a sound reflector or sound absorber with the combination of certain properties: numerical value of its density, degree of stiffness noticed by direct manipulation, its porosity observed with a binocular microscope.

31. Use a conceptual model of the microstructure to represent a dense material. 
32. Use a conceptual model that allows relating the density of a material with its acoustic behaviour.

33. Use a conceptual model of the microstructure to represent a rigid material.

34. Use a conceptual model that allows relating the rigidity of a material with its acoustic behaviour.

35. Represent a porous material in terms of its internal structure.

36. Use a conceptual model that allows relating the porosity of a material with its acoustic behaviour.

37. Use a conceptual model to interpret mechanisms of sound attenuation in materials in terms of energy transfer or energy dissipation.

FOR PART (A):

\begin{tabular}{|c|c|c|}
\hline & CATEGORY OF STUDENT'S RESPONSE & EXAMPLE OF STUDENT RESPONSE \\
\hline 1 & $\begin{array}{l}\text { Students correctly identify both the best sound } \\
\text { reflector and the best sound absorber taking into } \\
\text { account the description provided of the three } \\
\text { properties that affect the acoustic behaviour of } \\
\text { these materials. Therefore, students are able to } \\
\text { appropriately apply the conceptual model which } \\
\text { relates the acoustic behaviour of these materials to } \\
\text { their physical properties. }\end{array}$ & $\begin{array}{l}\text { Material B is the best sound absorber because it } \\
\text { has certain properties: it has a low density, it is } \\
\text { flexible and porous. These properties do not allow } \\
\text { the material to reflect sound a lot and absorb a } \\
\text { great part of the sound energy. Material } C \text { is the } \\
\text { best sound reflector because it combines three } \\
\text { essential properties: it is very dense and rigid and } \\
\text { is not porous. These properties make the material } \\
\text { reflect sound a lot and therefore, avoid a great part } \\
\text { of sound from being transmitted. }\end{array}$ \\
\hline 2 & $\begin{array}{l}\text { Students are able to appropriately apply the } \\
\text { conceptual model which relates the acoustic } \\
\text { behaviour of materials to their physical properties } \\
\text { and they also explain why these properties affect } \\
\text { the acoustic behaviour of these materials, } \\
\text { explaining the relationship between physical } \\
\text { properties and the internal structure of materials. In } \\
\text { some of these cases, students' explanations } \\
\text { evidence the use of the sound model as an entity. }\end{array}$ & $\begin{array}{l}\text { Material C is the best sound reflector because it } \\
\text { has high density. Therefore, its particles have more } \\
\text { inertia and they are less prone to be moved. } \\
\text { Moreover, the material is rigid and is less prone to } \\
\text { be deformed. Hence, its particles do not easily } \\
\text { move. Finally, it is not porous, therefore, sound } \\
\text { cannot enter into the material but is rather } \\
\text { reflected. Material B is the best sound absorber } \\
\text { because it has a low density and less inertia, } \\
\text { allowing sound propagation. It is flexible and can } \\
\text { be easily deformed. It is porous which allows it to } \\
\text { absorb sound inside the pores. }\end{array}$ \\
\hline 3 & $\begin{array}{l}\text { Students correctly identify both the best sound } \\
\text { reflector and the best sound absorber taking into } \\
\text { account the description provided from some of the } \\
\text { properties that affect the acoustic behaviour of } \\
\text { these materials. }\end{array}$ & $\begin{array}{l}\text { Material } B \text { is the best sound absorber because it is } \\
\text { porous. }\end{array}$ \\
\hline 4 & $\begin{array}{l}\text { Students incorrectly identify the acoustic behaviour } \\
\text { of each material taking into account some of the } \\
\text { described properties but not all. }\end{array}$ & $\begin{array}{l}\text { Material } C \text { is a sound absorber because it is not } \\
\text { very porous, so that it avoids sound transmission. }\end{array}$ \\
\hline
\end{tabular}


FOR PART (B):

\begin{tabular}{|l|l|l|}
\hline 1 & CATEGORY OF STUDENT'S RESPONSE & EXAMPLE OF STUDENT RESPONSE \\
\hline 1 & $\begin{array}{l}\text { Students' responses illustrate a clear distinction } \\
\text { between sound absorbers and sound reflectors } \\
\text { depending on the data of sound intensity level } \\
\text { measured inside three boxes covered with } \\
\text { different materials. Therefore, students compare } \\
\text { the values of sound intensity level inside the } \\
\text { different boxes with the reference value inside the } \\
\text { box. Moreover, students explain the acoustic } \\
\text { behaviour of each material relating sound intensity } \\
\text { level with the amount of energy associated to } \\
\text { sound waves. }\end{array}$ & $\begin{array}{l}\text { Material } D \text { is the best sound absorber because the } \\
\text { sound intensity level inside and outside the box is } \\
\text { lower than the reference value (inside the box), } \\
\text { which means that the material absorbs a great part } \\
\text { behaves as a sound absorber, it does not } \\
\text { attenuate as much sound as material } D \text {. Therefore, } \\
\text { we can say that material } D \text { is the best sound } \\
\text { absorber. Material E is the best sound reflector } \\
\text { because the sound intensity level inside the box is } \\
\text { higher than the reference value (inside the box), } \\
\text { which means that sound is amplified inside the } \\
\text { box; that is, the material reflects a lot. }\end{array}$ \\
\hline 2 & $\begin{array}{l}\text { Students identify (correctly or incorrectly) the } \\
\text { acoustic behaviour of materials in terms of the } \\
\text { sound attenuation that they produce. The idea that } \\
\text { sound absorbers allow more sound propagation } \\
\text { and so they attenuate less sound seems to be } \\
\text { related to the students' conception of sound as an } \\
\text { entity or substance that can go through materials. }\end{array}$ & $\begin{array}{l}\text { Material } D \text { is the best sound reflector because it } \\
\text { does allow sound transmission. This material is the } \\
\text { best sound insulator. }\end{array}$ \\
\hline
\end{tabular}

FOR PART (C):

\begin{tabular}{|l|l|l|}
\hline & CATEGORY OF STUDENT'S RESPONSE & EXAMPLE OF STUDENT RESPONSE \\
\hline 1 & $\begin{array}{l}\text { Student responses illustrate that sound attenuation } \\
\text { is appropriately conceptualized as the decrease of } \\
\text { the sound intensity level when sound is transmitted } \\
\text { through a certain material. Therefore, students } \\
\text { compare the values of transmitted sound (sound } \\
\text { intensity level outside the box covered with each } \\
\text { material) to determine which material attenuates } \\
\text { sound more or less. }\end{array}$ & $\begin{array}{l}\text { Material } D \text { is the best sound insulator and material } \\
F \text { is the worst. }\end{array}$ \\
\hline 2 & $\begin{array}{l}\text { Students do not appropriately identify the best and } \\
\text { worst sound insulator from the provided data. For } \\
\text { instance, some students consider that sound } \\
\text { attenuation is the difference between sound } \\
\text { intensity level inside a box covered with a certain } \\
\text { material and the reference value (inside the box). } \\
\text { Therefore, these student responses denote a lack } \\
\text { of understanding of what sound attenuation is. }\end{array}$ & $\begin{array}{l}\text { Material } D \text { is the best sound insulator because it } \\
\text { sound insulator because it only attenuates } 2 d B .\end{array}$ \\
\hline
\end{tabular}




\section{QUESTION 5}

A building company has developed a new generation of sound absorbers. The advertisement of this company says:

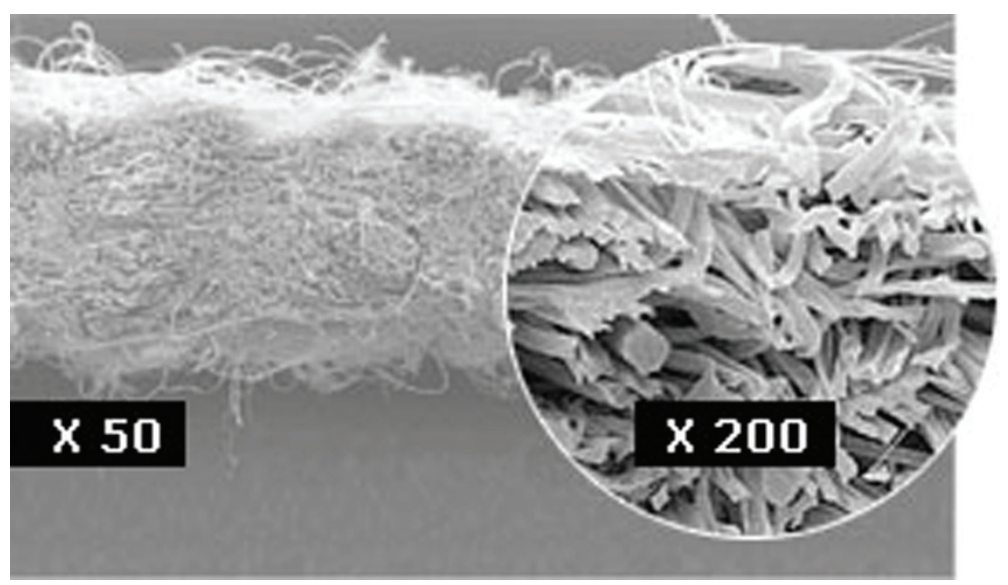

Absorson is a textile that offers very good sound absorbing properties. Its structure, made of fibres or microfilaments is very compact. It is very durable. This material is permeable to air and has a density of $0,2 \mathrm{~g} / \mathrm{cc}$.

Up to now, the materials used were very thick and heavy, but the structure of microfilaments of Absorson guarantees an efficient sound absorption with minimal thickness (approximately 0,5 $\mathrm{mm}$ ) and a density 10 to 30 times lower.

In addition, it is very flexible and it can be used in many different fields such as construction or the car industry. For instance, the installation of this material in a big restaurant reduced the sound intensity level up to $10 \mathrm{~dB}$.

(a) Only identify in the text the physical properties that influence the acoustic behaviour of the material and make it a good sound absorber.

(b) How would you explain the fact that these properties make this material a good sound absorber? Give reasons for your answer in terms of the internal structure of this material and in terms of the particles that form the material.

\section{Rubrics (Q5)}

Q5 is intended to assess whether students have achieved the following learning targets, that is, if they are able to:

30. Relate the fact that a material is a sound reflector or sound absorber with the combination of certain properties: numerical value of its density, degree of stiffness noticed by direct manipulation, its porosity observed with a binocular microscope.

31. Use a conceptual model of the microstructure to represent a dense material.

32. Use a conceptual model that allows relating the density of a material to its acoustic behaviour.

33. Use a conceptual model of the microstructure to represent a rigid material.

34. Use a conceptual model that allows relating the rigidity of a material with its acoustic behaviour.

35. Represent a porous material in terms of its internal structure.

36. Use a conceptual model that allows relating the porosity of a material with its acoustic behaviour.

37. Use a conceptual model to interpret mechanisms of sound attenuation in materials in terms of energy transfer or energy dissipation. 
FOR PART (A):

\begin{tabular}{|c|l|l|}
\hline 1 & CATEGORY OF STUDENT'S RESPONSE & EXAMPLE OF STUDENT RESPONSE \\
\hline 1 & $\begin{array}{l}\text { Students identify in the text of the advertisement } \\
\text { as properties of sound absorbers those which are } \\
\text { explicitly related to the conceptual model which } \\
\text { relates acoustic behaviour of materials and } \\
\text { physical properties: low density, porosity, low } \\
\text { rigidity. Therefore, these students only highlight the } \\
\text { properties of the material that affect acoustic } \\
\text { behaviour. }\end{array}$ & $\begin{array}{l}\text { The properties that affect the acoustic behaviour } \\
\text { of this material are low density, flexibility and } \\
\text { porosity since it is formed by filaments. Other } \\
\text { characteristics, such as the little thickness and } \\
\text { weight, are advantages highlighted for advertising } \\
\text { reasons but they are not relevant to make the } \\
\text { material good or bad sound absorber. }\end{array}$ \\
\hline 2 & $\begin{array}{l}\text { Student responses include other properties that } \\
\text { are attributes of a piece of material but are not } \\
\text { characteristics of a material. }\end{array}$ & $\begin{array}{l}\text { The properties that make this material a good } \\
\text { sound absorber are: permeability to air, internal } \\
\text { structure made of fibres and compactness. }\end{array}$ \\
\hline 3 & $\begin{array}{l}\text { Student responses emphasize any evidence in } \\
\text { the text that supports the acoustic behaviour } \\
\text { of the material or emphasizes other qualities of } \\
\text { the material, such as its range of application or its } \\
\text { durability, which are characteristics that make the } \\
\text { material good to be used as a product but do not } \\
\text { affect its acoustic behaviour. }\end{array}$ & $\begin{array}{l}\text { The property that affects the fact that a material is } \\
\text { a good sound absorber is low density (lower than } \\
\text { other materials' density). The text also tells the } \\
\text { example that this material decreased the sound } \\
\text { intensity level between } 6 \text { and 10 dB in a } \\
\text { restaurant. }\end{array}$ \\
\hline
\end{tabular}

FOR PART (B):

\begin{tabular}{|c|c|c|}
\hline & CATEGORY OF STUDENT'S RESPONSE & EXAMPLE OF STUDENT RESPONSE \\
\hline 1 & $\begin{array}{l}\text { Student responses explain the relationship } \\
\text { between physical properties (porosity, density and } \\
\text { rigidity) and internal structure of materials. } \\
\text { Moreover, these students use this relationship to } \\
\text { account for the acoustic behaviour of the sound } \\
\text { absorber mentioned in the activity. Therefore, } \\
\text { students explain the mechanisms of sound } \\
\text { attenuation or energy dissipation. }\end{array}$ & $\begin{array}{l}\text { The material is flexible and so, its particles are } \\
\text { linked in such a way that they can vibrate easily, } \\
\text { avoiding too much reflection and dissipate part of } \\
\text { the energy with this vibration. Moreover, the } \\
\text { material is porous. Therefore, the air particles filling } \\
\text { the pores inside this material vibrate transmitting } \\
\text { sound, and, therefore they rub and collide with the } \\
\text { solid material, provoking that part of the sound } \\
\text { energy is absorbed. Sound which propagates } \\
\text { inside this material is reflected many times } \\
\text { because of the changes of medium. Furthermore, } \\
\text { the material has a low density so that its particles } \\
\text { have little inertia, that is, they can vibrate easily. } \\
\text { Therefore, when sound reaches this material, it } \\
\text { avoids that great part of the sound that is } \\
\text { transmitted through it. }\end{array}$ \\
\hline 2 & $\begin{array}{l}\text { Student responses also explain the relationship } \\
\text { between physical properties and internal structure } \\
\text { of materials and the mechanisms of sound } \\
\text { attenuation or energy dissipation of sound } \\
\text { absorbers. Nevertheless, these responses } \\
\text { evidence a weak understanding of the nature of } \\
\text { sound, applying the "entity" model of sound. }\end{array}$ & $\begin{array}{l}\text { The fact that the material is made of fibres implies } \\
\text { that there are cavities with air between particles. } \\
\text { Sound can be saved in these cavities. The fact that } \\
\text { the material has a low density and is not very rigid } \\
\text { implies that the bonds between particles are not } \\
\text { very strong and so, the particles can vibrate and } \\
\text { transmit sound easily. }\end{array}$ \\
\hline 3 & Irrelevant or inconsistent answers & \\
\hline
\end{tabular}




\section{QUESTION 6}

In an architecture forum on the Internet, a boy explains his experience in order to help other people who have the same problem:

"Some months ago I had a problem of noise coming from my neighbour's house. Through the dividing wall (the one that separates their flat and mine) I could hear their television, their music, etc. I tried a solution that was proposed in a magazine: building a double wall of drywall with two plates of $13 \mathrm{~mm}$ and leaving a space of 45 $\mathrm{mm}$ among them. In this way, I managed to attenuate the noise a little but I did not solve the problem completely. So, I followed one of my friend's suggestions: putting stone wool between the two plates of drywall. After this, I can guarantee that I do not hear the television of my neighbours, or their music, or practically anything".

a) How would you explain that the first solution that the boy tested already allowed attenuating sound?

b) What was the role of the stone wool? How would you account for its acoustic behaviour in terms of its physical properties and internal structure?

\section{Rubrics (Q6)}

Q6 is intended to assess whether students have achieved the following learning targets, that is, if they are able to:

10. Interpret sound attenuation in materials in terms of energy distribution.

12. Express and apply in different contexts the diagram that describes that an incident sound on an interface is partly reflected, partly absorbed and partly transmitted through a material.

13. Relate the sound intensity level measured with the quantity of energy that is associated to the sound wave.

14. Associate the sound intensity level decrease through a wall or any other obstacle to sound attenuation.

30. Relate the fact that a material is a sound reflector or sound absorber with the combination of certain properties: density, stiffness, and porosity.

31. Use a conceptual model of the microstructure to represent a dense material.

32. Use a conceptual model that allows relating the density of a material with its acoustic behaviour.

33. Use a conceptual model of the microstructure to represent a rigid material.

34. Use a conceptual model that allows relating the rigidity of a material with its acoustic behaviour.

35. Represent a porous material in terms of its internal structure.

36. Use a conceptual model that allows relating the porosity of a material with its acoustic behaviour.

37. Use a conceptual model to interpret mechanisms of sound attenuation in materials in terms of energy transfer or energy dissipation. 
FOR PART (A):

\begin{tabular}{|l|l|l|}
\hline 1 & CATEGORY OF STUDENT'S RESPONSE & EXAMPLE OF STUDENT RESPONSE \\
\hline 1 & $\begin{array}{l}\text { Student responses illustrate a clear idea of the } \\
\text { phenomena that take place when sound reaches } \\
\text { an object. They explain these sound phenomena } \\
\text { in terms of energy distribution. Moreover, these } \\
\text { students are able to identify the acoustic behaviour } \\
\text { of the aforementioned materials and the physical } \\
\text { properties that affect such behaviour. }\end{array}$ & $\begin{array}{l}\text { When the man uses two plates of drywall } \\
\text { separated by a certain distance leaving air } \\
\text { between them, he is placing more obstacles to the } \\
\text { propagation of sound. Since the material is quite } \\
\text { rigid and is not porous, when sound coming from } \\
\text { the neighbours'house reaches the plates made of } \\
\text { this material is partly reflected. Moreover, the } \\
\text { plates made of this material also absorb a little part } \\
\text { of the energy of sound when propagating through } \\
\text { them. Therefore, more changes of medium } \\
\text { (drywall, air, drywall) in the path of sound imply } \\
\text { more sound attenuation (less sound intensity } \\
\text { level). }\end{array}$ \\
\hline 2 & $\begin{array}{l}\text { Students explain sound attenuation in terms of the } \\
\text { thickness of the object used. }\end{array}$ & $\begin{array}{l}\text { Placing two walls made of drywall and leaving a } \\
\text { space between them, the man achieves increasing } \\
\text { the thickness of the object used as an obstacle. } \\
\text { Therefore, the transmitted sound decreases. }\end{array}$ \\
\hline 3 & Irrelevant or inconsistent answers & \\
\hline
\end{tabular}


FOR PART (B):

\begin{tabular}{|c|c|c|}
\hline & CATEGORY OF STUDENT'S RESPONSE & EXAMPLE OF STUDENT RESPONSE \\
\hline 1 & $\begin{array}{l}\text { Students explain the acoustic behaviour of the } \\
\text { material in terms of its physical properties and } \\
\text { internal structure. Furthermore, these students } \\
\text { explain the mechanisms of sound attenuation that } \\
\text { take place when sound reaches this kind of } \\
\text { material. }\end{array}$ & $\begin{array}{l}\text { Stone wool is a porous material. It has a low } \\
\text { density and is quite flexible. These properties } \\
\text { make this material a good sound absorber. The } \\
\text { porosity allows a great part of the sound energy } \\
\text { reaching this material to be transferred to the } \\
\text { material without being reflected because sound } \\
\text { can continue propagating through the air inside the } \\
\text { pores of the material which are communicated with } \\
\text { the exterior. Sound waves that propagate through } \\
\text { the solid part of the material are partly reflected } \\
\text { and partly propagate within the material. The latter } \\
\text { part of sound finds several changes of medium } \\
\text { (from the solid part to the air and vice versa). } \\
\text { These waves are again partly reflected and partly } \\
\text { continue propagating, dissipating energy in each } \\
\text { reflection. Moreover, when air particles vibrate } \\
\text { inside the pores to transmit sound, they create } \\
\text { friction and collide against the solid part of the } \\
\text { material, dissipating part of the energy of sound. } \\
\text { Sound is absorbed within the material in this way. }\end{array}$ \\
\hline 2 & $\begin{array}{l}\text { Students explain the acoustic behaviour of the } \\
\text { material only in terms of its physical properties. }\end{array}$ & $\begin{array}{l}\text { Stone wool is a porous material. It has a low } \\
\text { density and is quite flexible. These properties } \\
\text { make this material a good sound absorber. }\end{array}$ \\
\hline 3 & $\begin{array}{l}\text { Students explain how this material behaves in front } \\
\text { of sound without relating it to its physical } \\
\text { properties. }\end{array}$ & $\begin{array}{l}\text { Stone wool is a good sound absorber and } \\
\text { attenuates sound a lot. }\end{array}$ \\
\hline 4 & Irrelevant or inconsistent answers & \\
\hline
\end{tabular}




\section{QUESTION 7}

Bill and Marie have two plates of different materials (A and B) and they want to know which material will attenuate sound more. The plate made of material $A$ has a thickness of $1 \mathrm{~cm}$ and the plate made of material $B$ has a thickness of $3 \mathrm{~cm}$. They have decided to cover the interior of the two boxes using the plates of each material. They will measure the sound attenuation produced by each box in order to draw some conclusions about which material attenuates more sound.

a) First of all, they want to determine whether the thickness of a plate of material affects the attenuation of sound produced by this plate. What experimental design would you suggest?

b) After carrying out the designed experiment, they obtained the following results:

\begin{tabular}{|c|c|}
\hline THICKNESS OF THE PLATE $(\mathbf{c m})$ & SOUND INTENSITY LEVEL OUTSIDE THE BOX (dB) \\
\hline 2 & 60 \\
\hline 3 & 55 \\
\hline 4 & 52 \\
\hline 5 & 50 \\
\hline
\end{tabular}

Which conclusion would you get from these results?

c) In order to test which material attenuates sound more, Bill and Marie discuss the experimental setting that they need to perform the experiment:

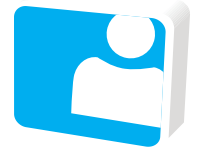

Bill: "I would cover the first box with plates of material A that have a thickness of $1 \mathrm{~cm}$ and the other box with plates of material B that have a thickness of $3 \mathrm{~cm}$ "

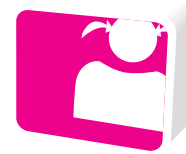

Marie: "I think that we should cover both boxes with plates that have the same thickness. Therefore, we should cover one box with three plates of material A and the other box with one plate of material $\mathrm{B}$. When we have prepared the boxes, we should put a constant sound source inside each box and also we should place the sound level meter inside the box. In this way, we can measure how much each material attenuates"

Do you agree with Bill and Marie? Do you agree with both of them or with only one of them? Explain your answer.

d) Would you modify the experiment in order to determine exactly which material (A or B) is the best sound insulator?

\section{Rubrics (Q7)}

Q7 is intended to assess whether students have achieved the following learning targets, that is, if they are able to:

16. Design an experiment to measure the attenuation of sound caused by a material.

17. Relate the sound intensity level measured in a certain point of the space with the distance from that point to the sound source.

18. Control the variables of an experiment.

25. Compare numerical values obtained empirically in order to come to some conclusions. 
FOR PART (A):

\begin{tabular}{|c|l|l|}
\hline & CATEGORY OF STUDENT'S RESPONSE & EXAMPLE OF STUDENT RESPONSE \\
\hline 1 & $\begin{array}{l}\text { Student responses illustrate an accurate control of } \\
\text { variables when designing and experimenting. } \\
\text { Moreover, students explain the procedures clearly } \\
\text { to measure sound attenuation. }\end{array}$ & $\begin{array}{l}\text { I would cover two (or more) boxes with plates } \\
\text { made of the same material (A or B) but the plates } \\
\text { of each box should have different thickness. Firstly, } \\
\text { I would measure a reference value of the sound } \\
\text { intensity level outside a box without being covered } \\
\text { by any plate. Later, I would measure the sound } \\
\text { intensity level transmitted through each of the } \\
\text { boxes (placing the sound level meter outside the } \\
\text { box at a fixed distance). }\end{array}$ \\
\hline 2 & Irrelevant or inconsistent answers & \\
\hline
\end{tabular}

FOR PART (B):

\begin{tabular}{|c|l|l|}
\hline CATEGORY OF STUDENT'S RESPONSE & EXAMPLE OF STUDENT RESPONSE \\
\hline 1 & $\begin{array}{l}\text { Students draw appropriate conclusions on the } \\
\text { empirical relationship between thickness of } \\
\text { material and sound attenuation produced from } \\
\text { quantitative data. }\end{array}$ & $\begin{array}{l}\text { The thicker the plate is the more sound it } \\
\text { attenuates because the lower values of sound } \\
\text { intensity are obtained when the box is covered with } \\
\text { thicker plates of material. }\end{array}$ \\
\hline 2 & Irrelevant or inconsistent answers & \\
\hline
\end{tabular}

FOR PART (C):

\begin{tabular}{|l|l|l|}
\hline & CATEGORY OF STUDENT'S RESPONSE & EXAMPLE OF STUDENT RESPONSE \\
\hline 1 & $\begin{array}{l}\text { Student responses evidence the importance } \\
\text { attributed to the control of variables in an } \\
\text { experiment. In addition, these responses denote } \\
\text { appropriate procedures for measuring sound } \\
\text { attenuation, which are closely related to what } \\
\text { students understand as sound attenuation. }\end{array}$ & $\begin{array}{l}\text { I do not agree with any of them. } \\
\text { Bill does not propose an appropriate experiment } \\
\text { because he would modify the thickness of the } \\
\text { plates and the material of the plate at the same } \\
\text { time. Therefore, he could not determine whether } \\
\text { differences of sound attenuation are due to } \\
\text { different thickness or to different material. Marie } \\
\text { would measure the sound intensity level inside the } \\
\text { box. Therefore, she could not measure sound } \\
\text { attenuation but she could determine if the material } \\
\text { attenuates because it reflects a lot or because it } \\
\text { absorbs a lot. }\end{array}$ \\
\hline 2 & $\begin{array}{l}\text { Student responses evidence the importance } \\
\text { attributed to the control of variables in an } \\
\text { experiment. However, these responses do not } \\
\text { denote appropriate procedures for measuring } \\
\text { sound attenuation. }\end{array}$ & \begin{tabular}{l} 
I agree with Marie. \\
\hline 3
\end{tabular} \\
\begin{tabular}{l} 
Irrelevant or inconsistent answers \\
\hline
\end{tabular}
\end{tabular}


FOR PART (D):

\begin{tabular}{|l|l|l|}
\hline & CATEGORY OF STUDENT'S RESPONSE & EXAMPLE OF STUDENT RESPONSE \\
\hline 1 & $\begin{array}{l}\text { Student responses demonstrate the importance } \\
\text { attributed to the control of variables in an } \\
\text { experiment. In addition, these responses denote } \\
\text { appropriate procedures for measuring sound } \\
\text { attenuation, which are closely related to what } \\
\text { students understand as sound attenuation. }\end{array}$ & $\begin{array}{l}\text { Marie correctly modifies the experiment proposed } \\
\text { by William because she suggests keeping } \\
\text { constant the thickness of the plates and varying } \\
\text { the type of material to cover the boxes in order to } \\
\text { determine which one is a better insulator. } \\
\text { Regarding the experiment proposed by Marie, the } \\
\text { measurements to be taken should be different. We } \\
\text { should measure a reference value of the sound } \\
\text { intensity level transmitted though a box without } \\
\text { being covered by any plate of material. Later, we } \\
\text { should measure the intensity level of transmitted } \\
\text { sound through each of the boxes covered with } \\
\text { different materials having placed a sound source } \\
\text { inside the box. The material which has produced } \\
\text { the higher decrease of intensity level of transmitted } \\
\text { sound would be the best sound insulator. }\end{array}$ \\
\hline 2 & Irrelevant or inconsistent answers & \\
\hline
\end{tabular}




\section{QUESTION 8}

The company DSR is working on a project involving soundproofing and acoustic conditioning of the local multipurpose building of the drawing.

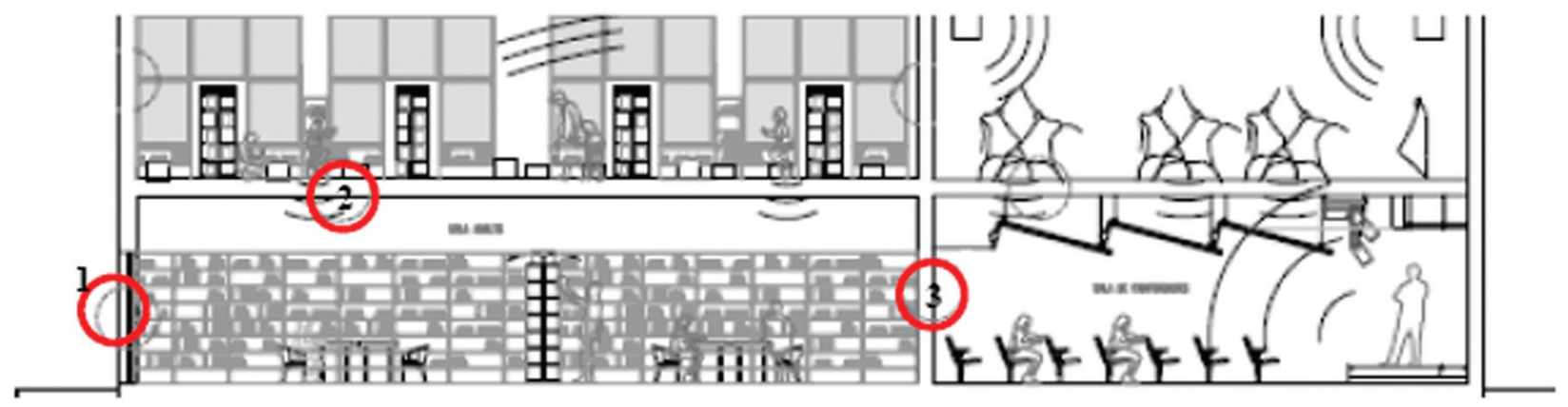

In the ground floor there is a library and a conference room (equipped with a good loudspeakers system) and in the first floor there is a dance room and a playing room for children. The entrance to the building is located at the library.

(a) Which acoustic conditions do you think the library should have to allow addressing an ordinary activity inside?

(b) In the previous image there are some points that could be problematic regarding noise inside the library. These are marked with a red circle. Explain why each of those points is problematic regarding the soundproofing (1- Entrance from the street, 2- Structure that separates the ground and the first floor, 3Dividing wall between the library and the conference room).

(c) To solve these problems, the company DSR proposes using different materials so that the sound intensity level inside the library is adequate to allow reading and studying. What characteristics do you think these materials should have in each of the problematic places identified? Explain your answer.

\section{Rubrics (Q8)}

Q8 is intended to assess whether students have achieved the following learning targets, that is, if they are able to:

3. Relate one's own experiences related to sound and noise pollution with scientific knowledge.

38. Apply the conceptual model which relates the physical properties of materials to their acoustic behaviour in order to predict how new materials would behave with regard to sound attenuation.

39. Describe materials.

43. Explain, using acoustic and non-acoustic criteria, their decisions about which materials are appropriate to use in order to achieve the acoustic conditions that a certain space requires.

44. Propose solutions to solve a problem related to soundproofing.

FOR PART (A):

\begin{tabular}{|c|l|l|}
\hline CATEGORY OF STUDENT'S RESPONSE & EXAMPLE OF STUDENT RESPONSE \\
\hline 1 & $\begin{array}{l}\text { Students appropriately identify the acoustic } \\
\text { conditions that are characteristic of a room } \\
\text { allocated for certain functions. }\end{array}$ & $\begin{array}{l}\text { The library requires low sound intensity levels so } \\
\text { that people can read and study peacefully. } \\
\text { Therefore, it should be appropriately soundproofed } \\
\text { to avoid sound propagating into this space. }\end{array}$ \\
\hline 2 & Irrelevant or inconsistent answers & \\
\hline
\end{tabular}


FOR PART (B):

\begin{tabular}{|l|l|l|}
\hline & CATEGORY OF STUDENT'S RESPONSE & EXAMPLE OF STUDENT RESPONSE \\
\hline 1 & $\begin{array}{l}\text { Students appropriately identify the acoustic } \\
\text { problems associated with different spaces. }\end{array}$ & $\begin{array}{l}\text { In the library, sound coming from outside should } \\
\text { be avoided. For this reason, point } 1 \text { is problematic } \\
\text { because there is the door which connects to the } \\
\text { street. Point 2 is problematic because it } \\
\text { corresponds to the ceiling through which sound } \\
\text { produced by children playing and running in the } \\
\text { first floor can propagate towards the library. Point } \\
\text { 3is problematic because it corresponds to the wall } \\
\text { which separates the library and the conference } \\
\text { room through which very loud sounds emitted by } \\
\text { the loudspeakers can propagate towards the } \\
\text { library. }\end{array}$ \\
\hline 2 & Irrelevant or inconsistent answers & \\
\hline
\end{tabular}

FOR PART (C):

\begin{tabular}{|c|c|c|}
\hline & CATEGORY OF STUDENT'S RESPONSE & EXAMPLE OF STUDENT RESPONSE \\
\hline \multirow[t]{3}{*}{1} & $\begin{array}{l}\text { Students suggest appropriate solutions to solve } \\
\text { the acoustic problems previously identified. } \\
\text { Moreover, they relate acoustic behaviour of } \\
\text { different materials to their physical properties. }\end{array}$ & $\begin{array}{l}\text { Point 1: it would be advisable that walls and doors } \\
\text { were thick, were made of a material which is very } \\
\text { dense, rigid and non porous, that is, sound } \\
\text { reflectors which avoids noise from the street to } \\
\text { propagate into the library. }\end{array}$ \\
\hline & & $\begin{array}{l}\text { Point 2: the floor of the playing room should be } \\
\text { covered with a flexible, low density and porous } \\
\text { material that allows absorbing a great part of the } \\
\text { energy of the sound produced by children. In the } \\
\text { ceiling of the library sound absorbing materials } \\
\text { could also be used as well. }\end{array}$ \\
\hline & & $\begin{array}{l}\text { Point 3: the walls of the conference room should } \\
\text { be covered with materials which reflect sound, but } \\
\text { also with materials that absorb part of the sound to } \\
\text { avoid too much reverberation inside this room. In } \\
\text { this way, only a little part of sound emitted by the } \\
\text { loudspeakers of the conference room would reach } \\
\text { the library. }\end{array}$ \\
\hline 2 & Irrelevant or inconsistent answers & \\
\hline
\end{tabular}




\section{D: DESCRIPTION OF}

MODULE DESIGN, DEVELOPMENT AND VALIDATION PROCESS 


\section{THE CONSORTIUM OF THE LWG}

The development of the module has been done by the Local Working Group (LWG), in a university-school partnership.

The participants from the university are:

- National coordinator of the project (Roser Pintó)

- Coordinator of the LWG (Digna Couso)

- Research assistant (Marisa Hernández)

From the 4 schools involved, 6 teachers have participated actively during the whole design process:

- 2 teachers (Montse and Consol) from School A (state school)

- 2 teachers (Celsa and Carme) from School B (state school)

- 1 teacher (Marta) from School C (state school)

- 1 teacher (Miquel) from School D (subsidised private school)

\section{DESIGN OF A TEACHING / LEARNING SEQUENCE ON ACOUSTIC PROPERTIES OF MATERIALS}

Theoretical Approach

The design of the sequence on acoustic properties of materials took into account different principles according to epistemological frameworks that we regard as complementary. Both the Developmental Research paradigm (Lijnse 1995) and the Model of Educational Reconstruction (Duit, Gropengießer \& Kattmann 2005) have been considered as powerful theoretical frameworks to support the process of design and validation of TLS.

From the Developmental Research framework, we highlight the importance of designing didactical structures (Lijnse 1995) where motivational and cognitive variables such as students' ownership of the learning process and metacognition are integrated. We considered these elements when designing the TLS on acoustic properties of materials.

The Model of Educational Reconstruction states that the analysis of both scientific contents and students' learning needs are equally important for developing a new and more efficient teaching approach. From this perspective, research and innovation should be paired activities. According to this model, the scientific content structure to be taught needs to be reconstructed from an educational perspective.

Taking this into consideration, the first stage in the process of development of the activity sequence that we carried out was the analysis of the contents of the subject matter, that is, the clarification of the subject matter and the analysis of plausible educational significance for pupils at 14-16 years. This process of subject matter clarification included analysis of publications on the topic coming from different fields: acoustics, engineering, architecture, physics, and materials science. That is, a long process of didactical transposition (analysing the scientific contents from the domain of Materials Science, Physics, and Chemistry to become knowledge for instruction) took place.

The revision of previous research studies about students' pre-instructional conceptions on nature and propagation of sound was also regarded as an important element for the design. From this perspective, a preliminary research study (Hernández, 2008) of students' conceptions on sound attenuation and acoustic properties of materials was carried out and the corresponding findings were taken into account when designing the sequence for structuring the conceptual sequence and designing certain activities. Some of the results of this study are:

- Most of the students from the studied sample expressed the idea that materials that attenuate sound behave as a sound barrier that avoids the passage of sound. This kind of conceptualization could imply an underlying idea of sound as a physical entity that can go or not through a material depending on certain characteristics that it possesses. It corresponds to the common conception described as the "entity model" of sound (see part 8 in section A). Other students recognized sound absorption as a phenomenon related to sound attenuation even though they could not explain it in terms of energy transfer.

- The different properties that students assign to materials according to their acoustic behaviour depend or are based on their conception of sound and its attenuation. That is, depending on what students figure out that sound and sound attenuation means, they consider that certain 
properties (and not others) of a material influence its capacity of attenuating sound. For instance, some students conceptualized sound attenuation as the opposition to the propagation of sound through a material. Some of these students expressed the idea that materials that attenuate sound more are denser and non porous to avoid the passage of sound though them.

- Some of the students' answers that described the relationship between properties of materials and their microstructure also suggested a weak understanding or simplification of the meaning of the considered properties. For instance, some students associated the density of materials with the distance between particles.

Appraising the differences between the school science contents to be taught and the students' conceptualizations on this area we could identify possible learning demands (Leach \& Scott 2002) for students in the last years of compulsory secondary school. From this analysis, we could formulate the following school science teaching goals: (i) Students should build a model which explains/justifies how materials behave in front of sound and how they attenuate sound. This implies, elaborating a model of sound reflector and sound absorber, which relates sound attenuation with physical properties or microstructure of materials; (ii) Students also need a clear understanding of the meaning of properties of materials at both macroscopic and microscopic level; (iii) As the concept of sound attenuation needs a strong basis on the concept of sound, it is necessary to improve students' understanding of the nature and propagation of sound in order to scaffold their building of an appropriate model of sound attenuation.

\section{DEVELOPMENT AND REFINEMENT OF THE SEQUENCE}

During the process of designing the activity sequence, the members of the LWG actively collaborated in periodical face-to-face meetings and also using an online platform. In an initial stage, a preliminary structure of the sequence was decided among the LWG members. The learning targets to be attained, the scientific contents to be taught (once the didactical transposition took place) and the introduction of pedagogical innovations (mainly the inquiry approach and the experimental setting) were proposed by the researchers. Some purposeful meetings were devoted to the introduction of these innovative ideas for teaching. Teachers also provided very useful remarks about student possibilities and real classroom contexts. In short, teachers and researchers were united at the core of designing the activities and the assessment tasks of the sequence.

After having designed the sequence, we wondered how to evaluate its quality. With this purpose, we decided to establish learning targets for guiding the evaluation tasks of each phase of the sequence, taking into account the learning demands. These learning targets were the ones expected from students as a result of their participation in the implementation of the sequence, and therefore they were used to evaluate the effectiveness of the designed sequence. We interpreted the effectiveness of an innovation as a high degree of students' learning achievement. For this reason, in order to make an in-depth evaluation of the sequence, the students' learning outcomes were compared with the learning targets formulated at the design stage.

After the design phase, the first version of the sequence became completed. The teachers who participated in the process of design of the sequence also committed themselves to implement the sequence in their science classes. Before the implementation, each teacher decided the learning targets that s/he would emphasize so that the most of his/her students reached them, according to certain criteria such as the features of their class groups and the number of available hours to be devoted to the implementation.

The evaluation process was carried out in three main stages:

a) During the first implementation phase, two external experts undertook a peer review study visit and provided useful feedback for revising the sequence.

b) After the first classroom implementation, all the members of the LWG evaluated jointly the sequence and considered some changes on the basis of difficulties perceived by teachers in their implementation and by researchers during classroom observations. 
c) Some months after the first implementation, researchers carried out a comparison between the established learning targets and the students' learning outcomes.

This first analysis allowed proposing different kinds of changes of the sequence or teaching strategies in order to assess the effectiveness of the sequence. As a result of this stage, a second version of the sequence was developed and implemented once again in science courses. After this second classroom implementation, teachers and researchers proceeded to revise the re-elaborated sequence in the basis of the new evidences obtained.

\section{IMPLEMENTATION(S) OF THE SEQUENCE}

As was previously explained, some of the teachers who participated in the first classroom implementation trial of the sequence also implemented the second version of the sequence. Moreover, two new teachers who did not participate in the design process but were colleagues of two of the previous teachers also joined the LWG and used the teaching and learning materials implementing the second version in their science classes.

\section{EVALUATION OF THE SEQUENCE}

The implementation of the sequence allowed us to evaluate to what extent it is effective in promoting students' achievements regarding the intended learning targets. Therefore, the evaluation process has consisted of analysing the learning outcomes achieved by students and comparing them with the intended ones. According to it, the sequence has been refined. The teachers who collaborated in the design of the activity sequence and implemented it also suggested changes in the sequence, taking into account:

In order to evaluate the sequence, different data was collected by means of different agents: teachers and CRECIM team.

\section{From Teachers Involved in the Design and the Sequence Implementation}

Teachers that participated in the design of the sequence collected data from:

a) The final test. It was conceived as an assessment instrument (post-test) for both researchers and teachers.

b) The implementation worksheets (see Annex 2) that each teacher wrote as a diary for each lesson. Teachers collected their perceptions about different aspects of the implementation (their intentions for each lesson activity, degree of autonomy that they provide to their students, concepts that have required further clarification, degree of interest shown for the students according to their questions or comments, conceptual and procedural students' difficulties identified when doing the activities, their difficulties trying to use a new pedagogical approach, their difficulties regarding the concepts, their difficulties managing the class in groups, preparing the experimental setting, etc.

c) The student activity sequence. These worksheets are also used for continuous assessment and for doing research related to some specific points.

\section{From the CRECIM team}

Different actions were planned by the researchers of the CRECIM in order to collect data about the sequence implementation:

a) The list of intended learning targets and corresponding rubrics that have been established by the CRECIM team and accepted by the teachers.

b) A class observation grid (see Annex 3) was designed to follow some particular aspects and procedures during the classroom implementation of the sequence. It is mostly focused in four aspects:

- The teaching inquiry approach that teachers use in their classes.

- The support of the modelling process provided by the teachers to the students when constructing the conceptual model which relates the properties of materials (density, rigidity, porosity) to their acoustic behaviour, and the conceptual model justifying such behaviours in terms of their internal structure.

- The kind of teaching strategies regarding the experimental work using ICT and other students' activities using data collected from tables or pictures.

- The kind of interactions among students and students-teacher since we are persuaded that the debates, the work of students in common is a very necessary "tool" for scaffolding students building of knowledge. 
In short, in order to draw conclusions on the effectiveness of the implementation of the sequence, different sources of data were considered:

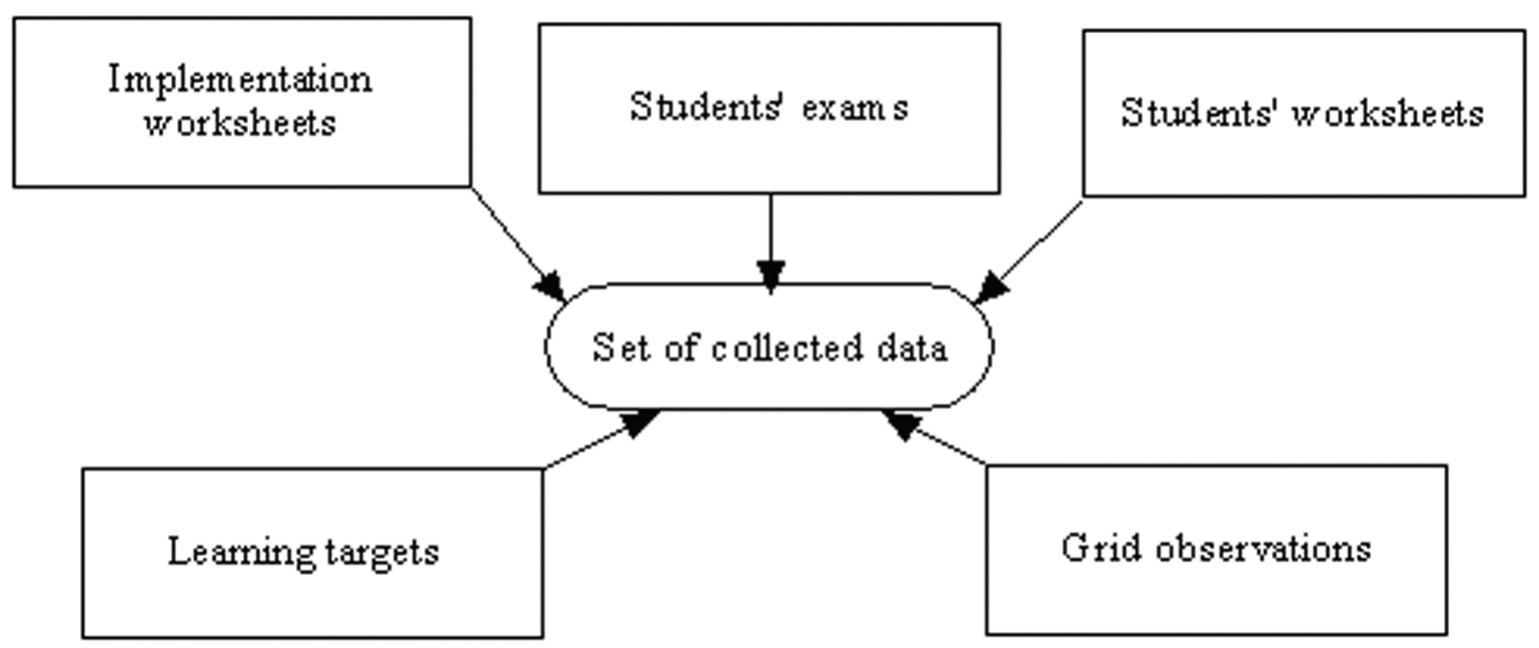

\section{ITERATIVE REFINEMENT OF THE SEQUENCE}

The iteration of the cycle design-implementationevaluation led to a refined sequence with enhanced coherence and more satisfactory learning outcomes on the part of students.

\section{References}

1. Hernández, M. I. (2008). Estudi de les concepcions dels estudiants de secundària sobre l'atenuació del so i les propietats acústiques dels materials. Non published minor thesis. Universitat Autònoma de Barcelona.

2. Leach, J. \& Scott, P. (2002). Designing and evaluating science teaching sequences: an approach drawing upon the concept of learning demand and a social constructivist perspective on learning. Studies in Science Education, 38, 3. Lijnse, P. (1995). "Developmental Research" as a way to an empirically based "Didactical Structure" of Science. Science Education, 79 (2), 189-199.

4. Duit, R., Gropengießer, H., \& Kattmann, U. (2005). Towards science education research that is relevant for improving practice: The model of educational reconstruction. In H.E. Fischer, Ed., Developing standards in research on science education (pp. 1-9). London: Taylor \& Francis. 



\section{ANNEX 1}




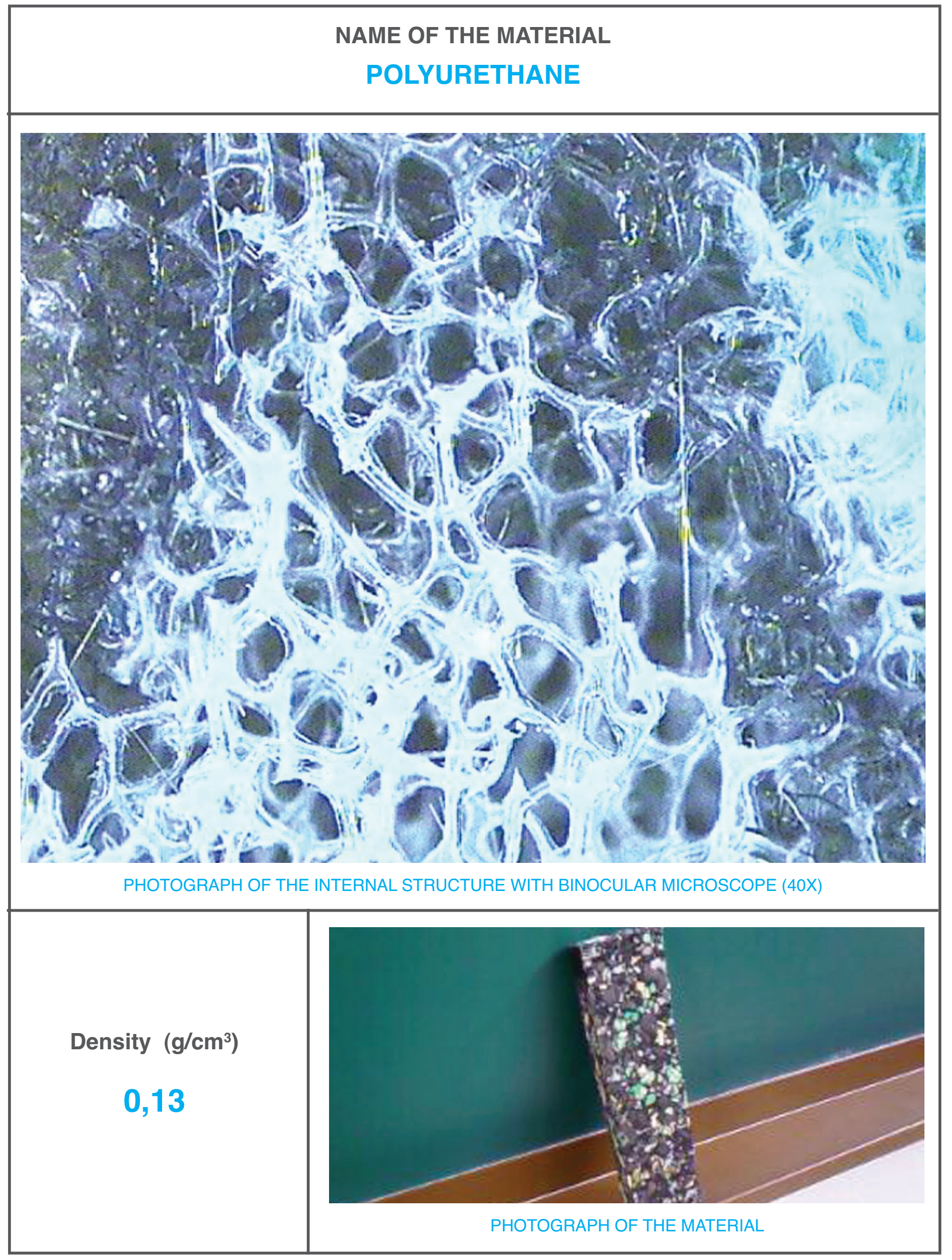




\section{NAME OF THE MATERIAL}

\section{FELT}

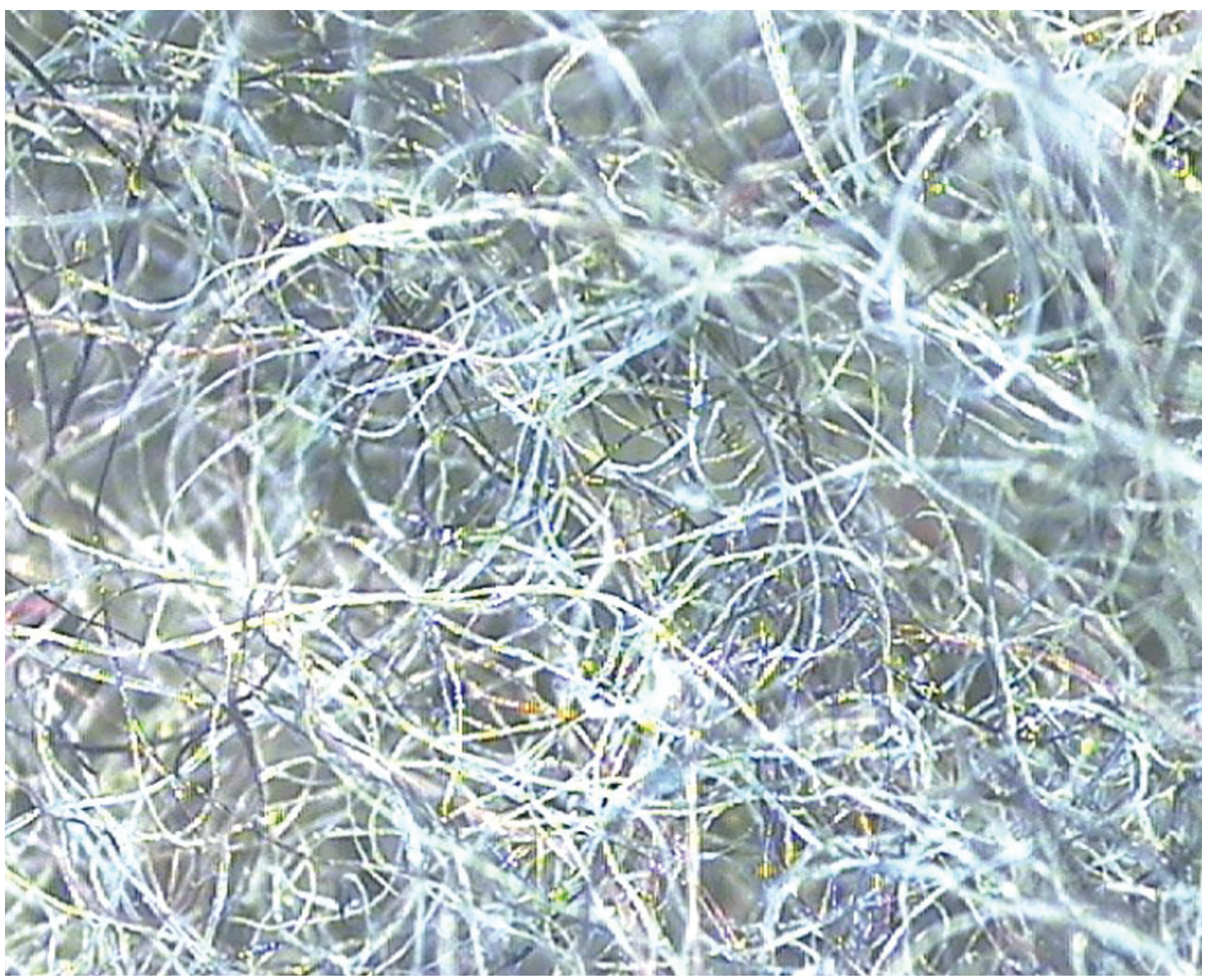

PHOTOGRAPH OF THE INTERNAL STRUCTURE WITH BINOCULAR MICROSCOPE (40X)

Density $\left(\mathrm{g} / \mathrm{cm}^{3}\right)$

0,07

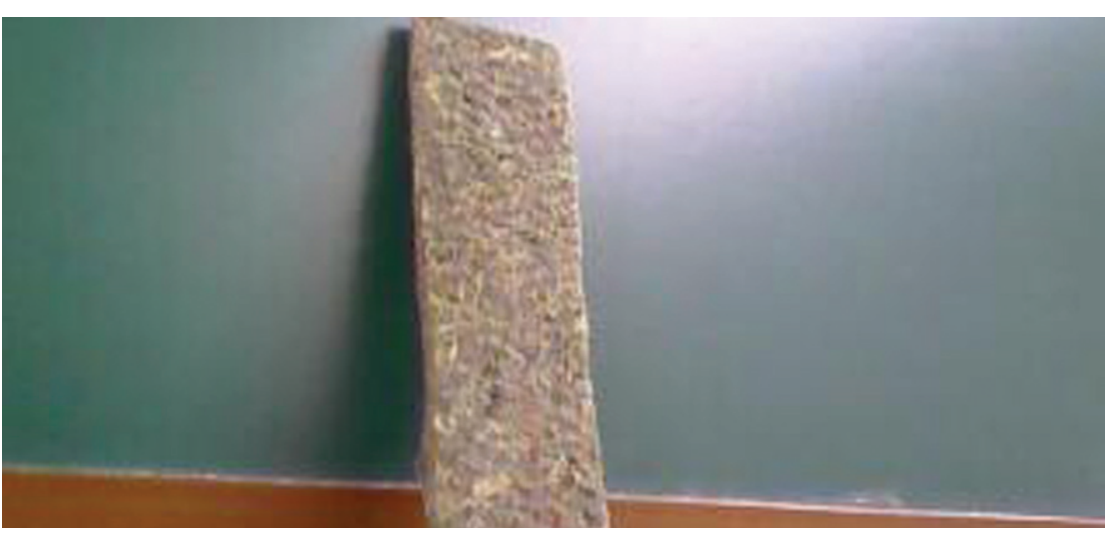

PHOTOGRAPH OF THE MATERIAL 


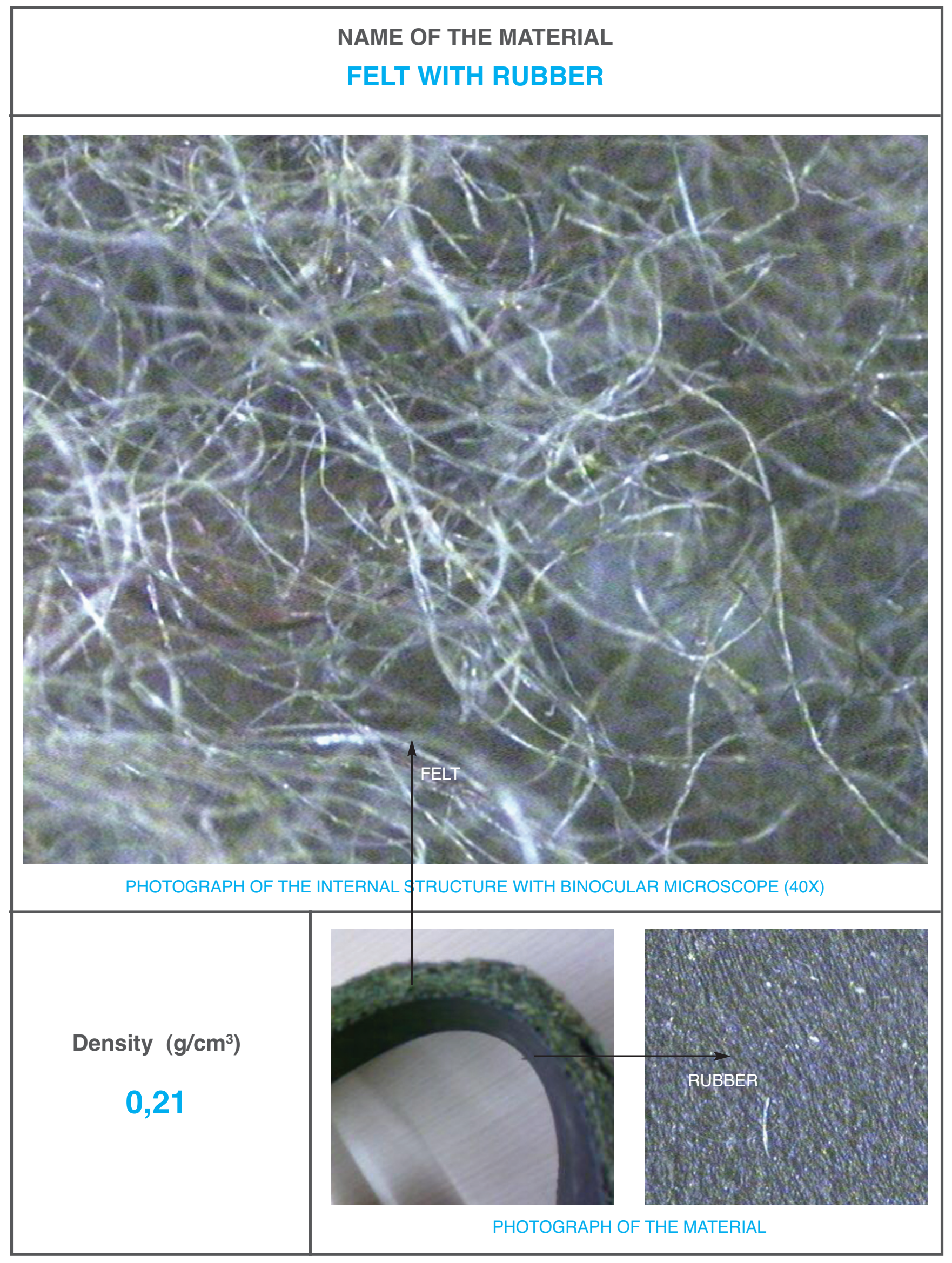




\section{NAME OF THE MATERIAL}

\section{ALUMINIUM FOIL}

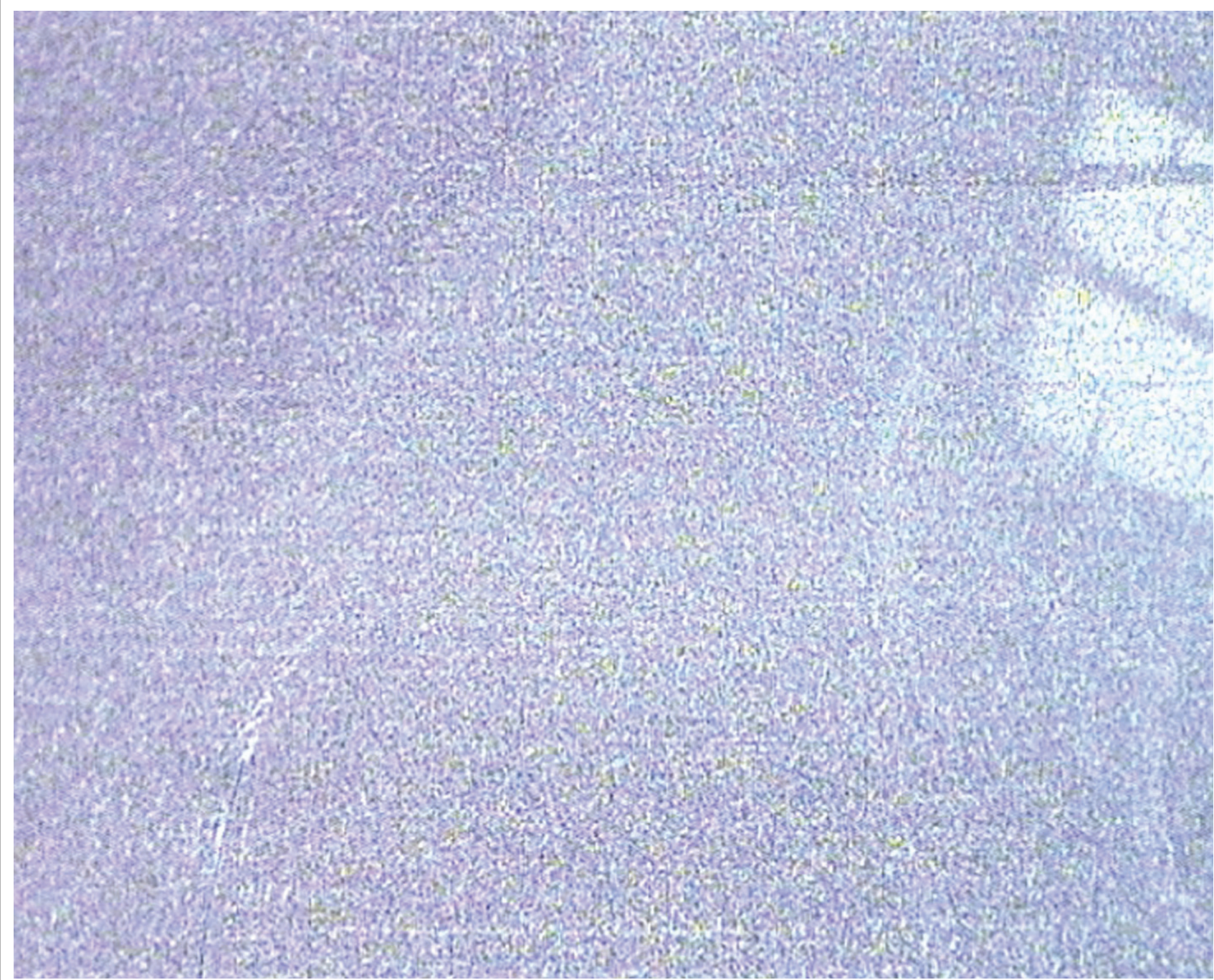

PHOTOGRAPH OF THE INTERNAL STRUCTURE WITH BINOCULAR MICROSCOPE (40X)

Density $\left(\mathrm{g} / \mathrm{cm}^{3}\right)$

2,70

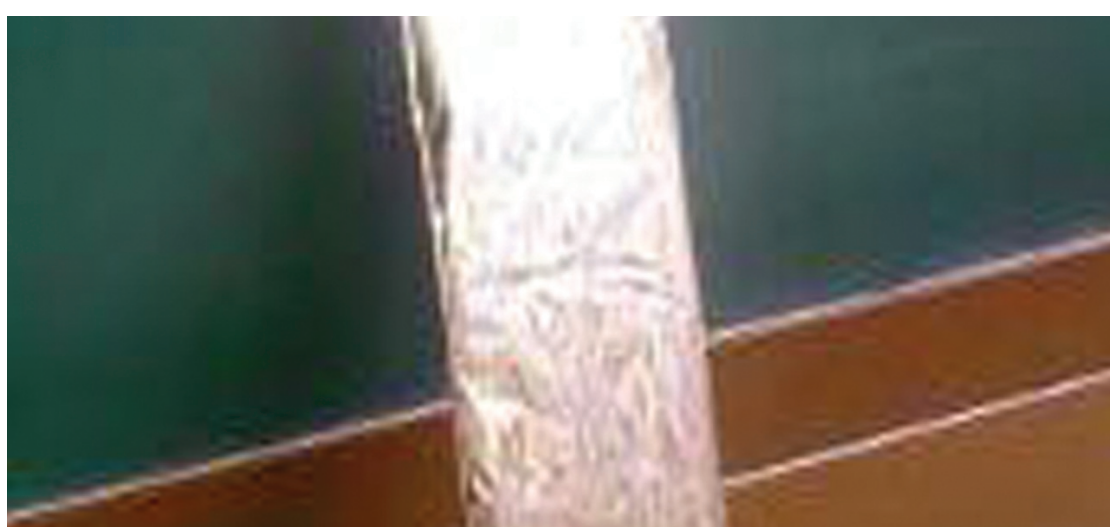

PHOTOGRAPH OF THE MATERIAL 


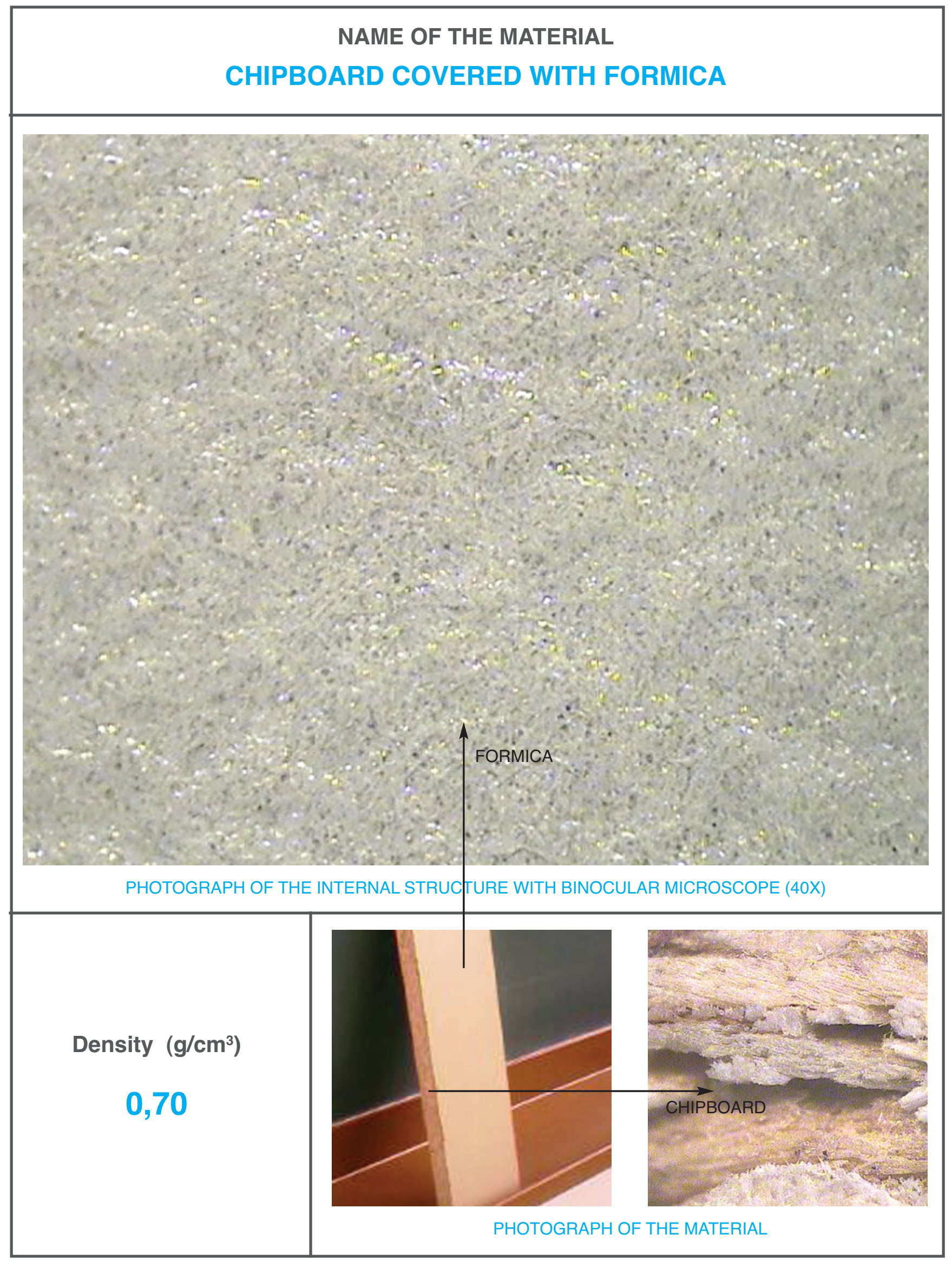




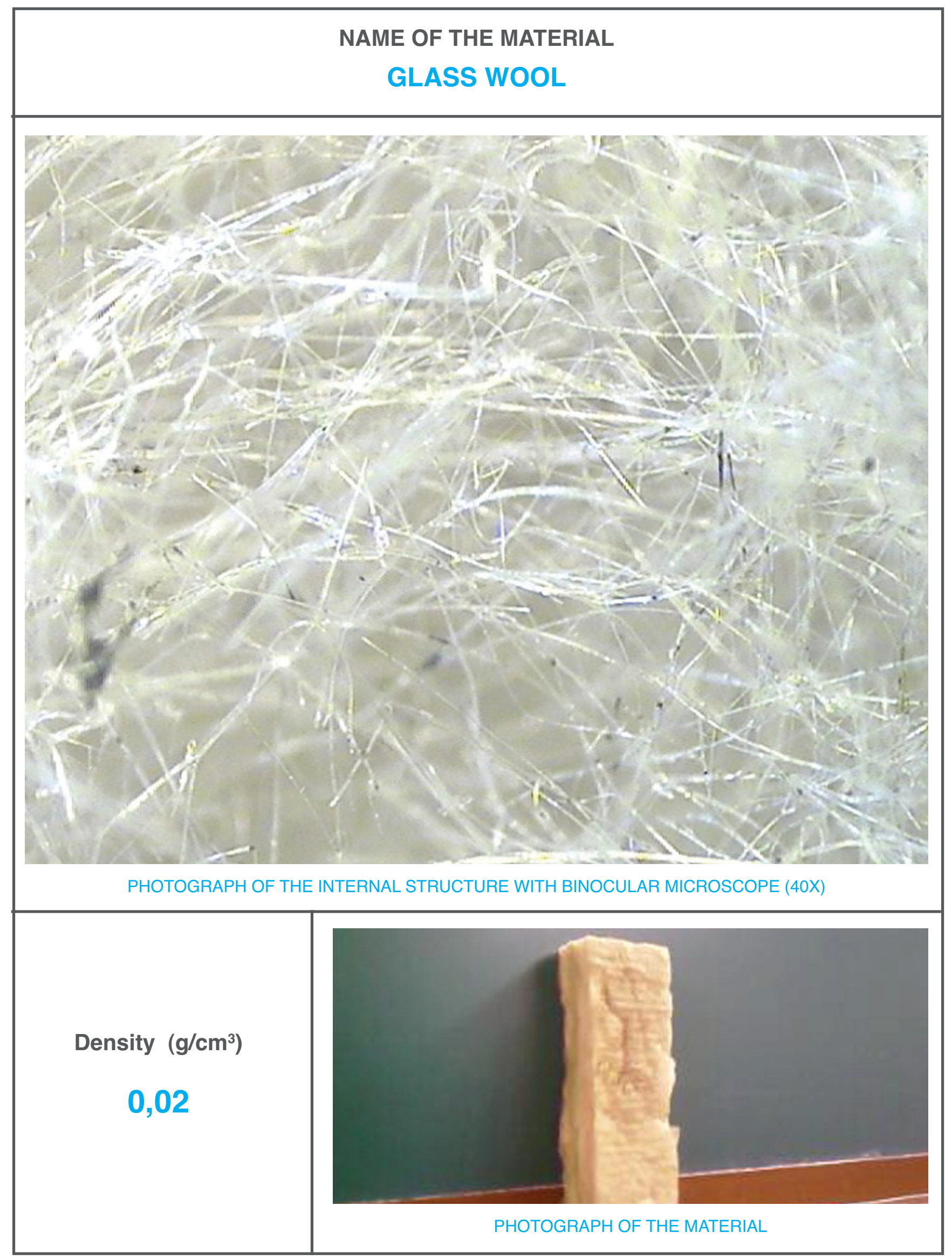




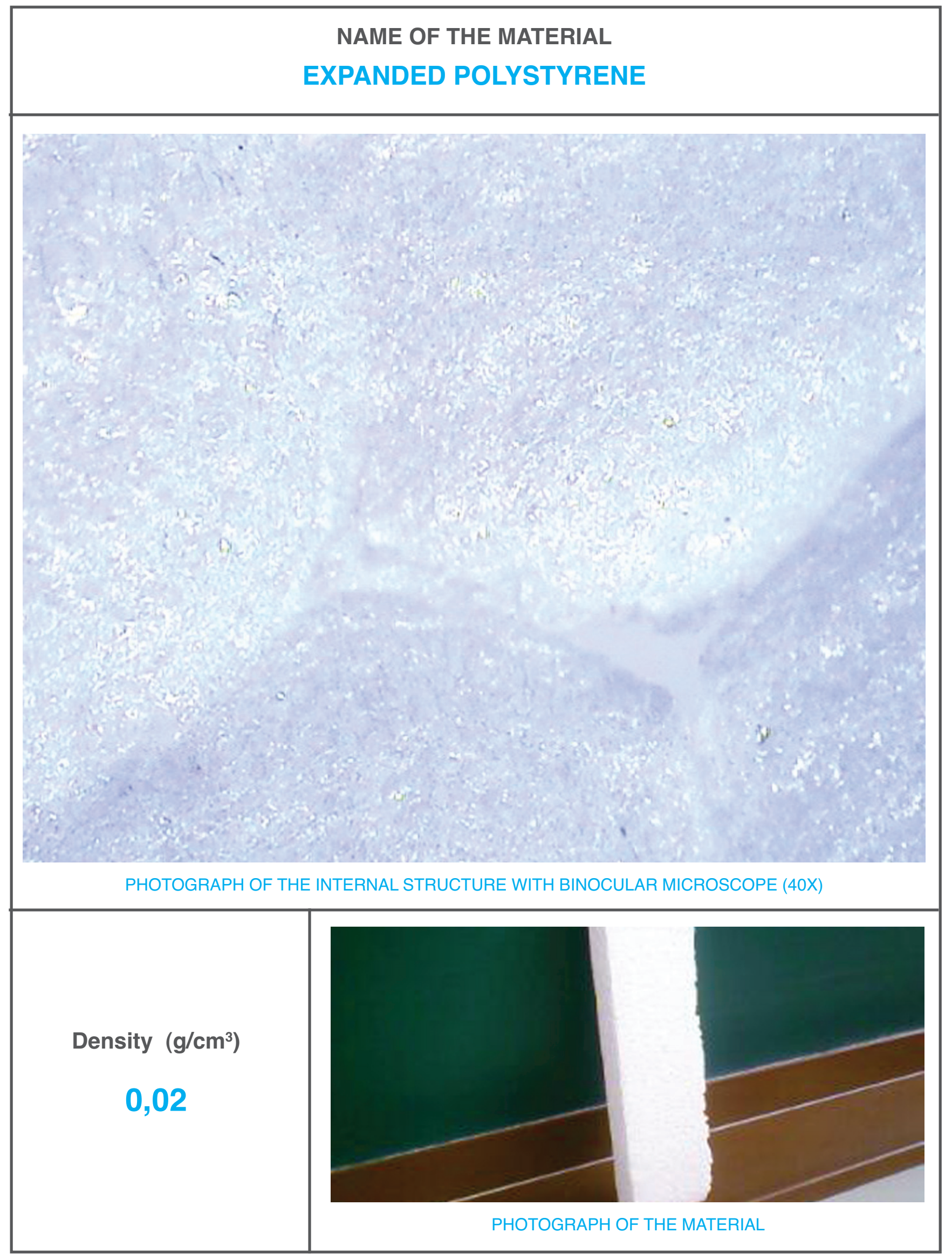




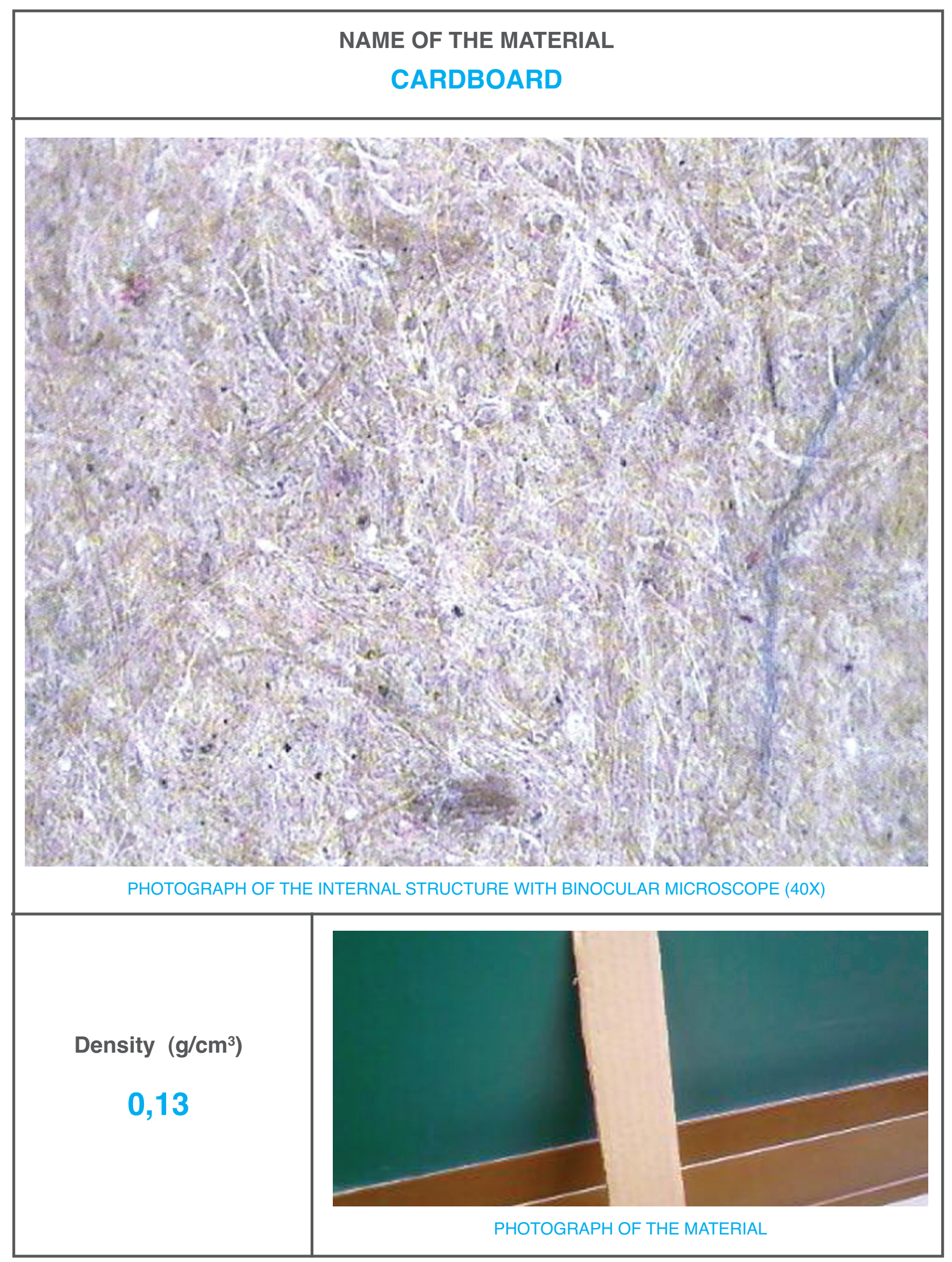




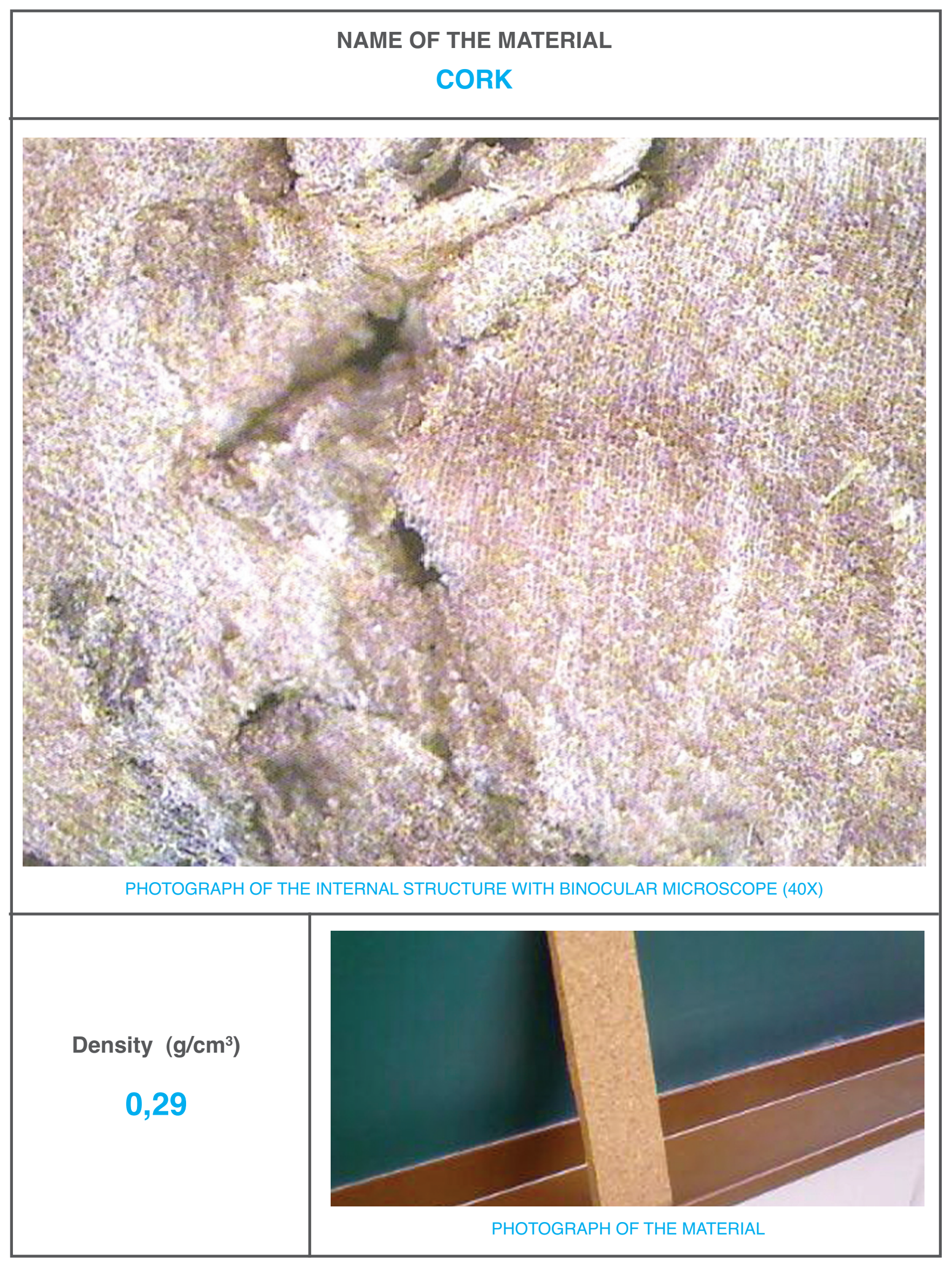




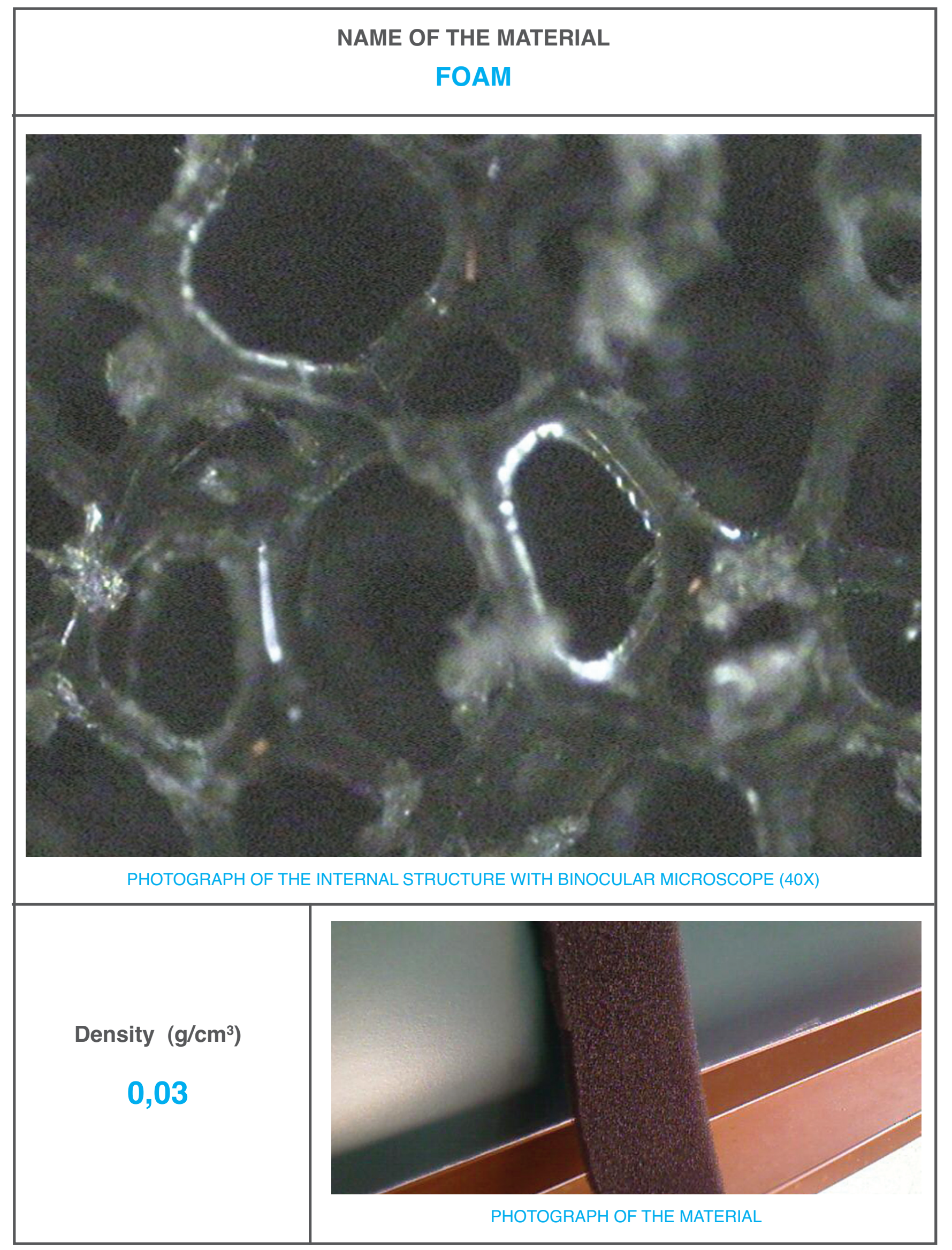




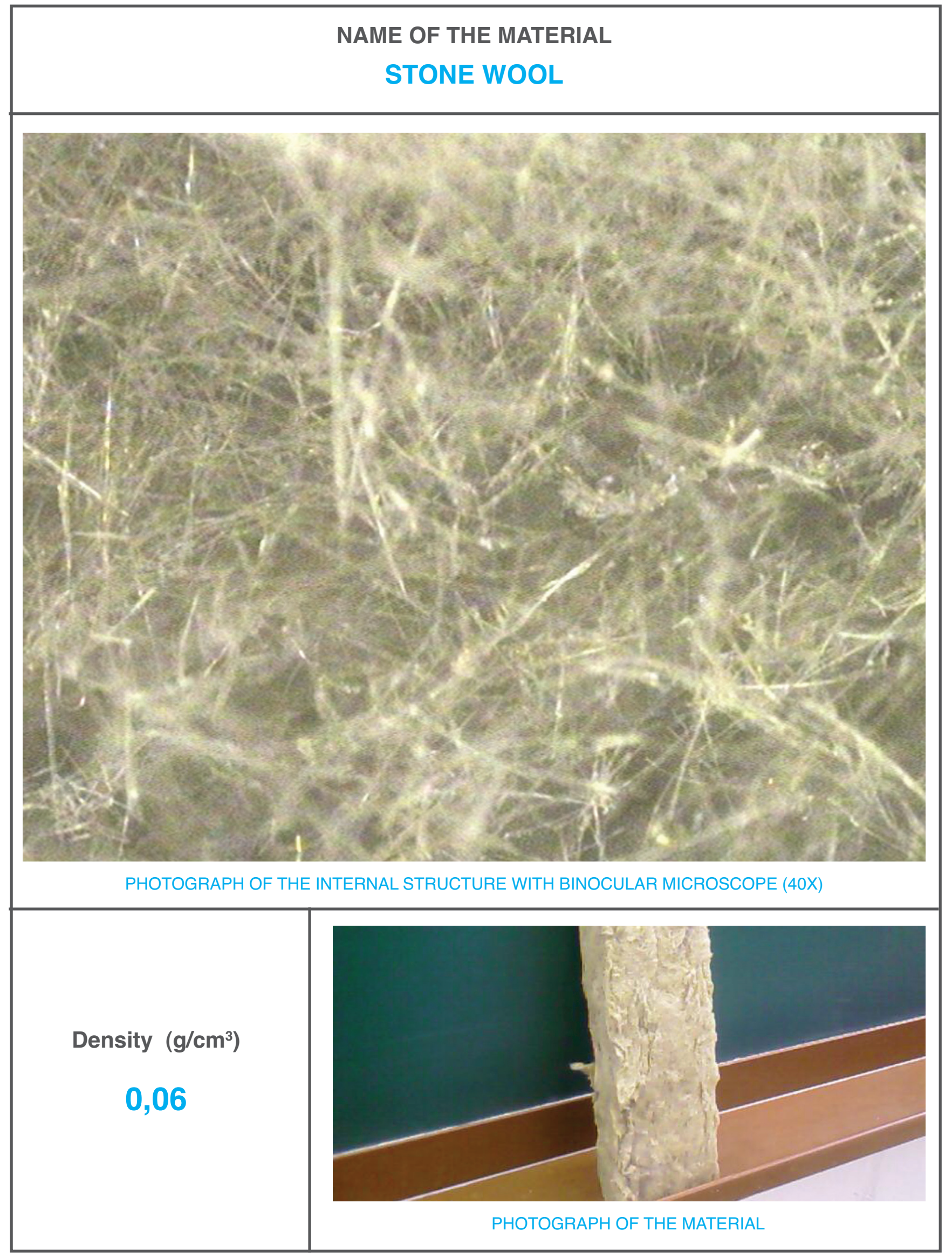


ANNEX 2: IMPLEMENTATION WORKSHEET 


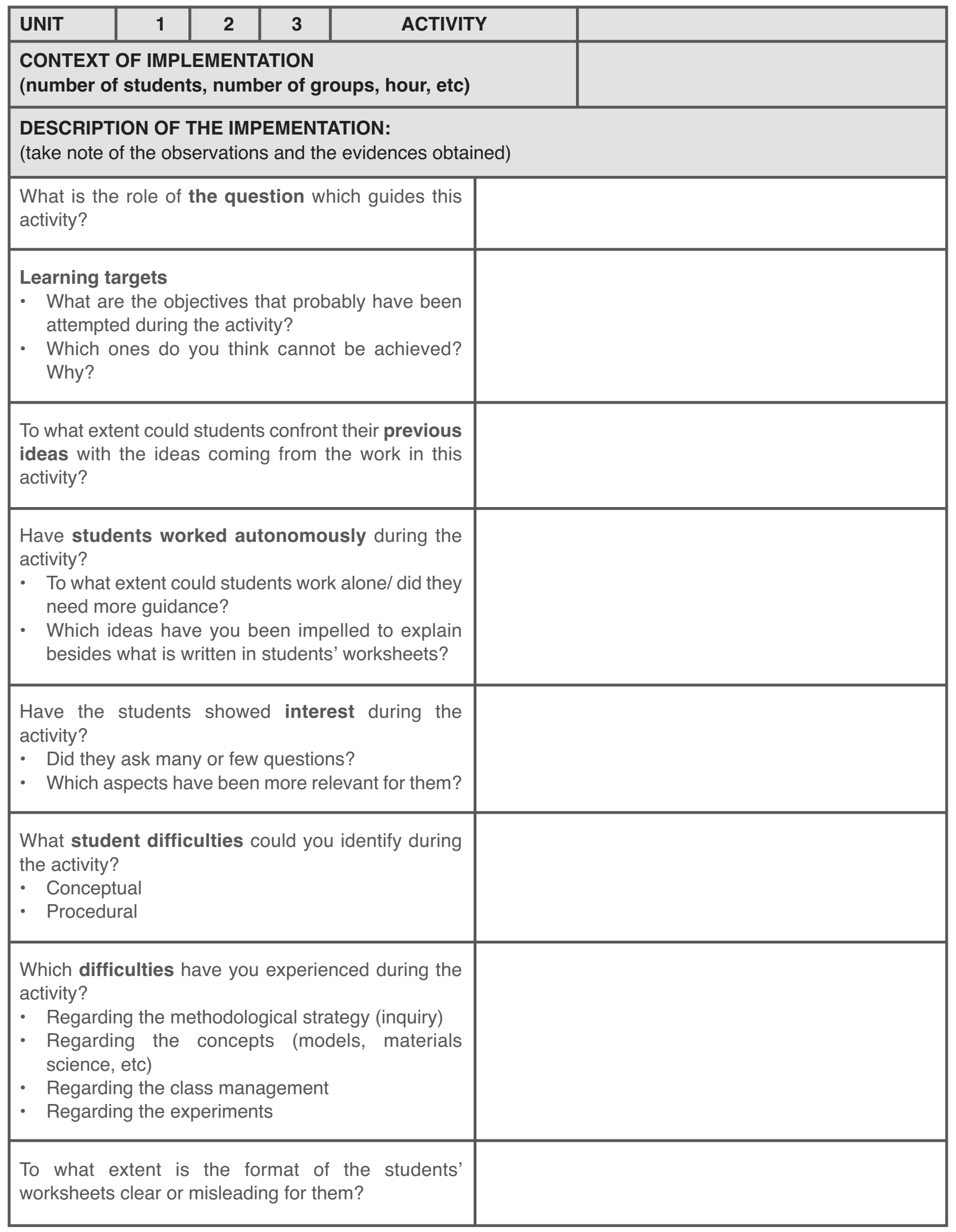


ANNEX 3: CLASS OBSERVATION GRID 


\section{TEACHING STRATEGIES REGARDING EXPERIMENTAL WORK AND ACTIVITIES PROPOSED}

Is the guiding question or purpose of the activity well identified by students?

How is the prediction of the experimental results proposed and done?

How do the students use the sound level meter and the MBL equipment?

How do students interpret the graphs? How do they assign a numerical value to sound intensity levels? Does the teacher need to help them to analyse or interpret the shape of the graph?

How are the students encouraged to make comparisons between experimental results and the predicted ones?

Are the students able to extract conclusions from qualitative data? How do they interpret the pictures and diagrams?

Is the equipment already prepared when the students arrive to the classroom?

\section{TEACHING STRATEGIES REGARDING THE INQUIRY APPROACH}

Do the students have the opportunity to answer questions or are they faced with some challenges?

Is it possible to notice that students understand/are quite sure about what they are trying to do, or what they are doing and what is expected from them?

(Internalisation of the objectives)

Does the teacher ask open ended questions frequently? (e.g., What are the evidences to infer such conclusions? Why have you decided such explanation/ argument instead of the previous one?) (Critical and logical thinking)

Do the students discuss data, evidences, etc in order to contrast their initial ideas?

Are students encouraged to design an investigation or to explore some idea by themselves?

(Degree of autonomy)

How much time is devoted to lecture with regards to the time assigned to dialogues or debates? 


\section{TEACHING STRATEGIES REGARDING THE MODELLING PROCESS}

How do students make the comparison between the previous ideas and the final conceptual model?

Is it possible to perceive if the new and more complex concepts are introduced having evidenced the limits of the previous models and the need for refinement?

Is it possible to perceive if the teacher try to make students build structures or nets of concepts instead of simple fragments of knowledge?

Does the teacher devote time to link ideas, synthesize explanations?

Are the students proposed to apply or transfer results to new situations?

\section{TEACHING STRATEGIES REGARDING STUDENT-STUDENT AND STUDENT-TEACHER INTERACTIONS}

Are the students distributed in groups or in pairs?

How is the feedback provided by the teacher to the students' comments?

Are the students working alone the time or are there some breaks according to the pupils needs?

How does the teacher follow the pupils' progress or difficulties? Does s/he use strategies to assess the progress of students during the session? 


MATERIALS

SCIENCE PROJECT

UNIVERSITY-SCHOOL PARTNERSHIPS

FOR THE DESIGN AND IMPLEMENTATION

OF RESEARCH-BASED ICT-ENHANCED

MODULES ON MATERIAL PROPERTIES

ISBN 978-9963-689-32-3

2009 